\title{
The mass of our Milky Way
}

\author{
Wenting Wang ${ }^{1,2 *}$, Jiaxin Han ${ }^{1,2 *}$, Marius Cautun ${ }^{3}$, Zhaozhou $\mathrm{Li}^{1}$, and Miho N. Ishigaki ${ }^{4}$ \\ ${ }^{1}$ Department of Astronomy, Shanghai Jiao Tong University, Shanghai 200240, China; \\ ${ }^{2}$ Kavli IPMU (WPI), UTIAS, The University of Tokyo, Kashiwa, Chiba 277-8583, Japan; \\ ${ }^{3}$ Leiden Observatory, Leiden University, PO Box 9513, NL-2300 RA Leiden, the Netherlands; \\ ${ }^{4}$ Astronomical Institute, Tohoku University, Aoba-ku, Sendai, Miyagi 980-8578, Japan
}

Received 000, 2019; accepted 000, 2019

\begin{abstract}
We perform an extensive review of the numerous studies and methods used to determine the total mass of the Milky Way. We group the various studies into seven broad classes according to their modeling approaches. The classes include: i) estimating Galactic escape velocity using high velocity objects; ii) measuring the rotation curve through terminal and circular velocities; iii) modeling halo stars, globular clusters and satellite galaxies with the Spherical Jeans equation and iv) with phase-space distribution functions; v) simulating and modeling the dynamics of stellar streams and their progenitors; vi) modeling the motion of the Milky Way, M31 and other distant satellites under the framework of Local Group timing argument; and vii) measurements made by linking the brightest Galactic satellites to their counterparts in simulations. For each class of methods, we introduce their theoretical and observational background, the method itself, the sample of available tracer objects, model assumptions, uncertainties, limits and the corresponding measurements that have been achieved in the past. Both the measured total masses within the radial range probed by tracer objects and the extrapolated virial masses are discussed and quoted. We also discuss the role of modern numerical simulations in terms of helping to validate model assumptions, understanding systematic uncertainties and calibrating the measurements. While measurements in the last two decades show a factor of two scatters, recent measurements using Gaia DR2 data are approaching a higher precision. We end with a detailed discussion of future developments in the field, especially as the size and quality of the observational data will increase tremendously with current and future surveys. In such cases, the systematic uncertainties will be dominant and thus will necessitate a much more rigorous testing and characterization of the various mass determination methods.
\end{abstract}

Milky Way, dark matter, stellar halo, dynamics, satellite galaxies

PACS number(s):

Citation: $\quad$ Wang W., Han J., et al, The mass of our Milky Way, Sci. China-Phys. Mech. Astron. 1, 000000 (2019), doi:

\section{Introduction}

In the current structure formation paradigm of $\Lambda$ cold dark matter $(\Lambda \mathrm{CDM})$, gas cools in the center of an evolving population of dark matter halos [398], which forms galaxies. Dark matter halos grow in mass and size through both smooth accretion of diffuse matter and from mergers with other halos [e.g. 389]. Smaller halos together with their own central galaxies fall into larger halos and become "subhalos" and "satellites" of the galaxy in the center of the dominant host halo. Orbiting around the central galaxy of the host halo, these satellites and subhalos lose mass due to tidal effects. Stars are stripped from them to form stellar streams, which then gradually mix in phase space. These stars form the stellar halo around the central galaxy [e.g. 1, 67, 82]. In the end, satellite galaxies and stripped material from these satellites merge with the central galaxy and contribute to its growth.

Compared with other distant galaxies, the distances and velocities of individual stars that form the diffuse stellar halo of our Milky Way (hereafter MW) can be directly observed, 
because we are embedded within our MW. The observed dynamics of luminous objects in the MW stellar halo, such as bright stars, satellite galaxies, globular clusters, maser sources, HI gas clouds and tidal streams, which serve as dynamical tracers, contain valuable information about the underlying potential. Given a reasonable model which describes their dynamics or phase-space distributions within a realistic potential profile with free parameters, one can constrain the total mass distribution and infer the total or virial mass of our MW.

We provide in Fig. 1 a literature summary of measured virial masses for the MW. It is an updated version of Figure 1 in Wang et al. [392] and Figure 7 in Callingham et al. [72]. The figure provides a general impression of the multitude of studies and the variety of methods used to constrain the virial mass of our Galaxy. For clarity, we grouped the various approaches into several categories, with each category shown in a different color. The figure shows only measurements with quoted statistical errors or confidence intervals, and does not include measurements without associated uncertainties. The exact $M_{200}$ values shown in Fig. 1 and their corresponding errors are provided in the second column of the table in Appendix A.

The measurements in Fig. 1 show a very large scatter. Part of the scatter is due to model extrapolations. For many of the studies, there were no or limited number of luminous tracers out to large enough distances, and thus to estimate the mass outside the radius of the most distant object, extrapolations of the model potential profile were made in these studies. For example, Taylor et al. in 2016 [372] reported that an accurate measurement of the mass within $50 \mathrm{kpc}$ can result in a $20 \%$ uncertainty on the virial mass of the Galaxy. Moreover, the virial mass plotted as the $x$-axis in Fig. 1 is defined as the total mass enclosed within a radius $R_{200}$, inside which the density is 200 times the critical density of the universe. The virial mass defined in this way is denoted as $M_{200}$. In fact, studies in Fig. 1 used varying definitions of virial masses. We have made conversions to change these different definitions to $M_{200}$, assuming that the underlying mass profiles follow the NFW halo mass profile [275, 276]. If the original studies have provided constraints for the halo concentration or relations to calculate the concentration, we take their concentration when making the conversion to $M_{200}$. Otherwise, we use a mean virial mass versus concentration relation provided by Duffy et al. in 2008 [110] to obtain the concentration and make the conversion. Additional uncertainties can be introduced through such conversions.

The remaining scatter in Fig. 1 is very likely caused by systematics in the models or peculiar assumptions when coping with incomplete data. For example, the velocity anisotropies for the observed tracer objects have to be known in order to break the mass-anisotropy degeneracy and properly constrain the rotation velocity or the underlying potential. However, tangential velocities in reality are often not available, and thus the velocity anisotropy has to be assumed as a constant, as a radius-dependent function with free parameters, inferred from numerical simulations or marginalized over, which unavoidably introduced additional uncertainties to the measured virial mass. Furthermore, many dynamical models rely on steady state and spherical assumptions, which might not be valid for our MW. Dynamically hot streams and coherent movements of satellite galaxies can violate the steady state assumption, and dark matter halos are triaxial [194].

In fact, many measurements in the past provided constraints on the circular velocities or the enclosed masses within the radii which can be covered by observed dynamical tracers, and we summarize these measurements in Fig. 2. In Appendix A, we provide in the third column of the table the enclosed masses within fixed radii, together with available circular velocities and local escape velocities at the solar radius, if these were provided. The readers can also find a similar figure from, for example, Eadie \& Jurić in 2019 [111], and a table from, for example, the review paper by BlandHawthorn et al. in 2016 [31]. The mass within the maximum radii of tracers should in principle be less model dependent and more reliable compared with the extrapolated virial mass in many cases. In fact, a general feature of dynamical modeling is that the best constrained mass for a given tracer is located around the median tracer radius [171, 387, e.g.].

Although the enclosed mass within a fixed radius, which is covered by the radial distribution of employed tracers, has less uncertainty than the extrapolated virial mass, the latter is still a very important and useful quantity in many applications. The virial mass is critical for comparing observed properties of the Milky Way with cosmological predictions. The so-called missing satellite problem [209, 267] is one of the examples. Very early on it was pointed out that the observed number of satellite galaxies is significantly lower than the predicted number of dark matter subhalos by numerical simulations. Although this problem can be alleviated by newly discovered faint MW satellites [e.g. 186, 187], explained by galaxy formation physics [e.g. 19, 68] which predicts that a significant number of small subhalos do not host a galaxy, or explained by warm dark matter which predicts significantly less number of small subhalos [e.g. 205, 249, 314], the total mass is closely related to the number of predicted subhalos [e.g. 130, 282]. A "light" MW contains fewer subhalos of a given mass and thus can help to alleviate the problem.

More recently, another problem, so-called "too big to fail", was raised by Boylan-Kolchin et al. in 2011 [54]. It concerns 


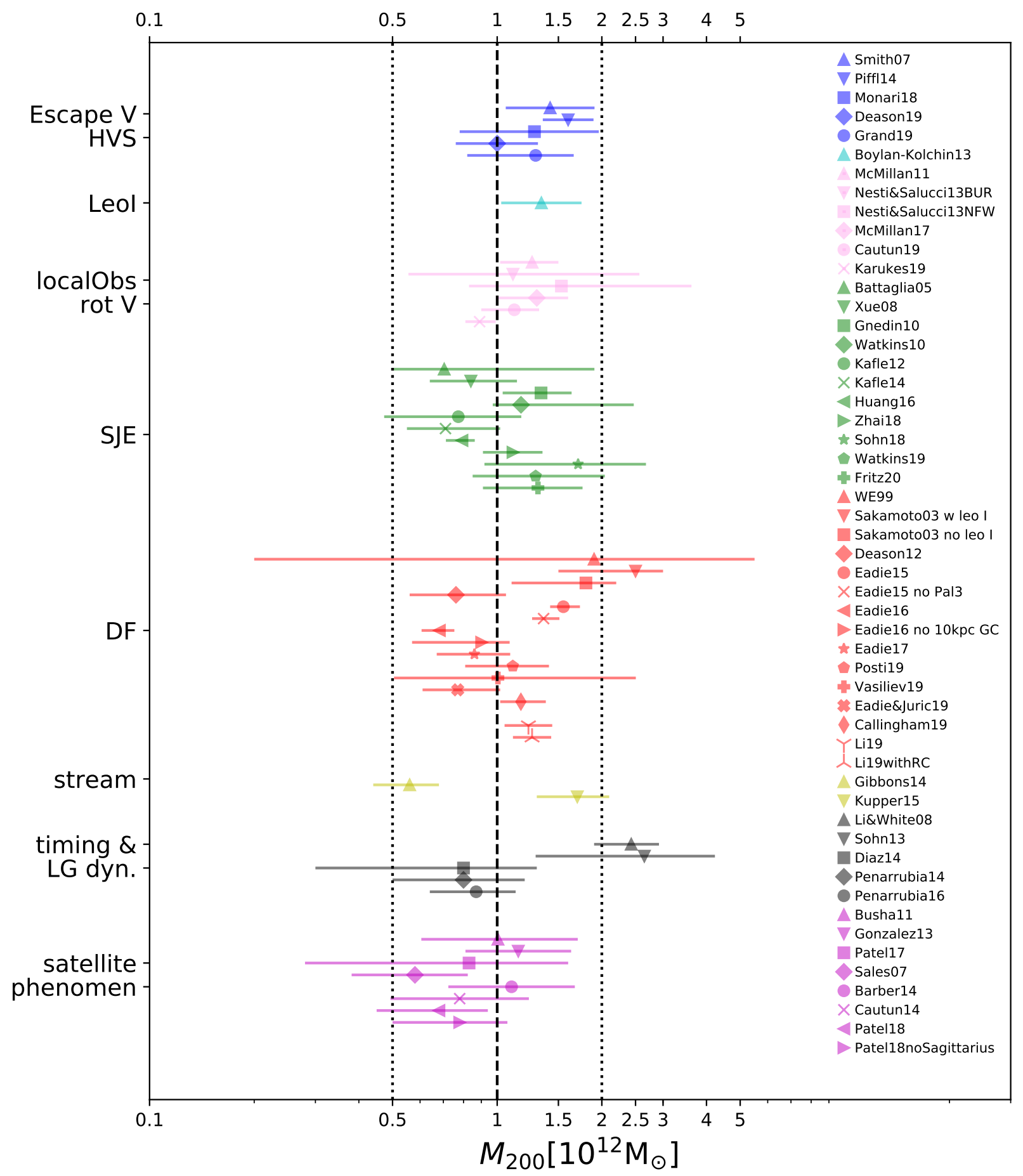

Figure 1 Literature compilation of inferred virial masses for the MW. Classes of methods are marked in different colors. Measurements have been converted to $M_{200}$, assuming NFW profiles. $95 \%$ or $90 \%$ confidence regions have been converted to $1-\sigma(68 \%)$ errors, assuming the errors are either Gaussian in linear space if the reported upper and lower errors have comparable size, or Gaussian in log space if the upper and lower errors have very different size in linear scale but are more comparable in log space. However, the assumption of Gaussian errors does not always hold. We just keep the original confidence regions $[111,113,114,115]$ or decrease the errors by about $10 \%$ for a few studies based on Bayesian analysis [279]. A few measurements have considered systematic uncertainties in their errors, for which we also keep the original errors [324, 393, 400]. The vertical dashed line at $1 \times 10^{12} \mathbf{M}_{\odot}$, and two vertical dotted lines at 0.5 and $2 \times 10^{12} \mathrm{M}_{\odot}$ are plotted to guide the eye. The readers can see Appendix A for a table summarizing these measurements, as well as the enclosed masses within fixed radii covered by tracer objects. A figure showing a subset of measurements using Gaia DR2 data are presented and discussed in Sec. 10 (Fig. 5). 


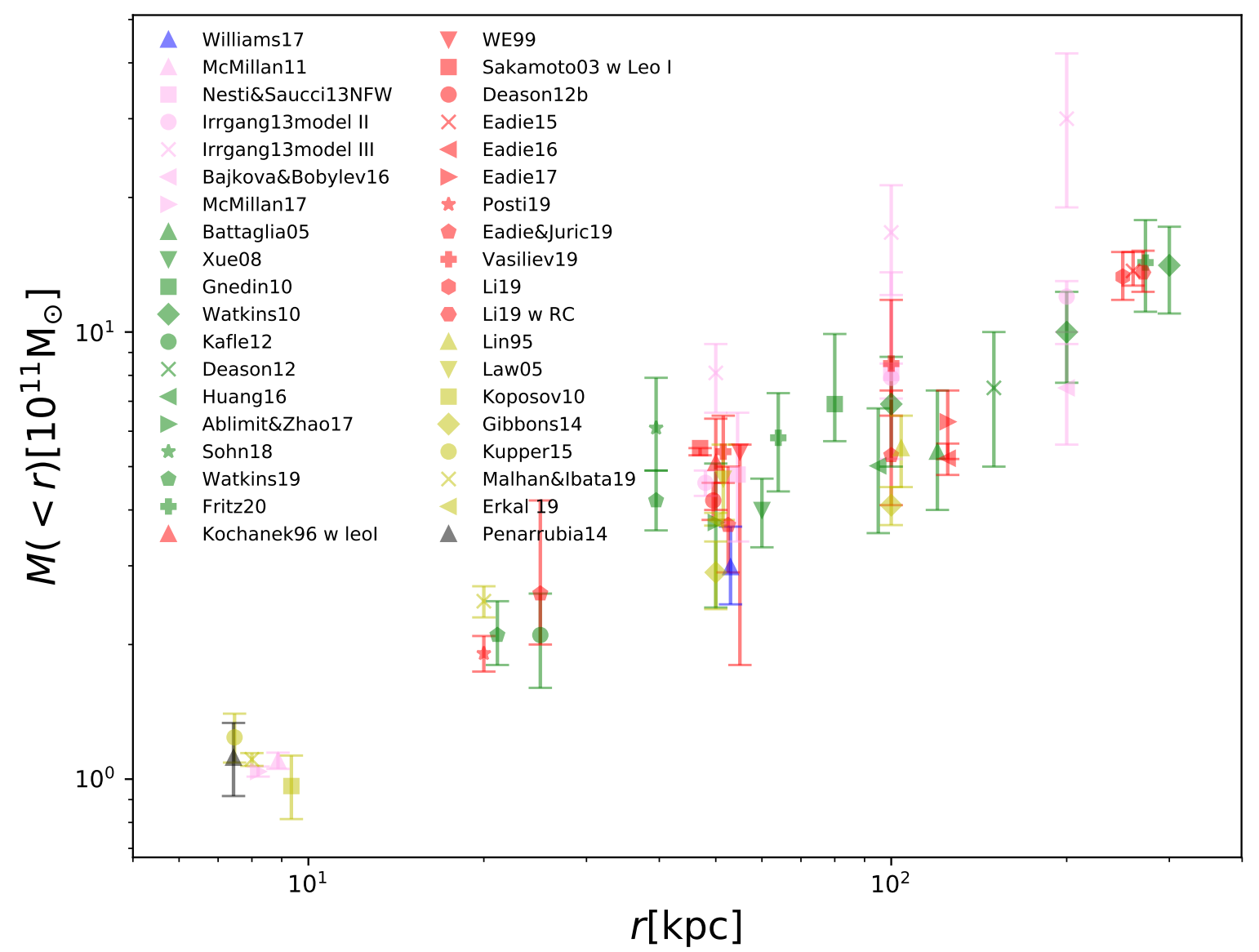

Figure 2 Literature compilation of enclosed masses within the radii which can be covered by observed dynamical tracers. The same color scheme as Fig. 1 is maintained for measurements grouped in the same category of method. Small offsets have been added to the groups of data points at $r=8,50,100$ and $260 \mathrm{kpc}$. 
an apparent lack of MW satellite galaxies with central densities as high as those of the most massive dark matter subhalos predicted by $\Lambda \mathrm{CDM}$ simulations of MW-like hosts. Proper mechanisms are required to explain how the central density of the most massive subhalos in simulations can be reduced in order to match observations. However, the number of massive halos in simulations strongly depends on the total mass of the MW, and the problem disappears if the mass of our MW is smaller than $1 \times 10^{12} \mathrm{M}_{\odot}$ [e.g. 80, 388].

The total mass and the underlying potential of our MW are also crucial for studies that focus on reconstructing the orbital evolution of individual objects. For example, it was discovered that MW satellite galaxies tend to be distributed in a highly inclined plane, and Pawlowski et al. [296] reported the discovery of a vast polar structure (VPOS) of satellite galaxies, globular clusters and streams around the MW, indicating anisotropic spatial distribution and infall of these objects. If planes of satellite galaxies are ubiquitous across the Universe, it poses great challenges to the standard cosmological model $[77,81]$. With more available proper motion data from Gaia DR2, it has become possible to look into details of the reconstructed orbits for these objects and examine whether they indeed move in the same plane [e.g. 136, 142, 349]. Such a study inevitably requires a fiducial potential model for the orbital integration. Any uncertainty in the potential model can affect the orbit integration and hence bias our understandings.

Knowing the MW mass is critical for predicting the future fate of our Galaxy, since having a more massive MW leads to a rapid merger of our Galaxy with its brightest satellite, the Large Magellanic Cloud (LMC) [78], and with its nearest giant neighbor, M31 [379].

Nowadays, we are in the large astronomical data survey era. Not only photometric and astrometric quantities, such as the magnitude, color, parallax (hence the Heliocentric distance) of each observed object can be measured, but also more and more objects with line-of-sight velocities, which approximately equal to the radial velocities for distant sources, have been collected through deep spectroscopic surveys, including the Radial Velocity Experiment [RAVE; 221, 368], the LAMOST ${ }^{1)}$ Experiment for Galactic Understanding and Exploration [LEGUE; 86, 101, 428], the Sloan Extension for Galactic Understanding and Exploration [SEGUE; 413], the Apache Point Observatory Galactic Evolution Experiment [APOGEE; 118], the Galactic Archeology with HERMES survey [GALAH 254] and the Gaia-ESO Survey [GES; 150]. Moreover, with high precision astrometric instruments, proper motions of stars can be measured [e.g. 378] by comparing imaging data taken at different epochs, after correcting for our own motion and controlling system- atics [e.g. 373]. More recently, with the launch of Gaia [301], a considerable amount of proper motion data are being collected. The mean proper motions of satellites and globular clusters based on their member stars have been refined and expanded [11, 136, 142, 202, 258, 290, 352, 383].

It is thus a good time to revisit the existing methodologies of measuring the total mass of our MW, and think about how to improve the modeling by better controlling systematic uncertainties and observational errors. Thus in this review, we provide detailed descriptions of existing methods measuring the total mass of our MW, the type of luminous objects which can be used as dynamical tracers of the underlying potential and modeling uncertainties. We hope to provide the reader better understandings towards these methods and broader views about how to make improvements in future studies. In addition, we hope our paper can help to summarize existing measurements for the mass of our MW in a clear and self-consistent way, and hence be useful for people who want to compare with these compiled measurements.

Note, however, although the baryonic mass makes an important contribution to the total mass of the inner MW, in this review we focus on methods of modeling and measuring the total mass. Details such as how to measure the mass in the nuclear region of the MW, stellar mass of the bulge and surface density in the local disk region through observations are beyond the scope of this review. The readers can check this information from the review paper of [31].

We start by introducing the method of measuring Galactic escape velocities using high velocity objects, in particular halo stars in the solar neighborhood (Sec. 2), and move on to introduce other local observables including terminal and circular velocities which can be used to measure the rotation curve for the inner MW (Sec. 3). Going further beyond the local observables, we introduce other methods including the spherical Jeans equation (Sec. 4) and the phase-space distribution function (Sec. 5), which model more distant dynamical tracer objects including halo stars, globular clusters and satellite galaxies. We describe the dynamical modeling of tidal streams in Sec. 6. Sec. 7 introduces the Local Group timing argument and the local Hubble flow approach by modeling mainly the radial motion of MW versus M31, and the motion of more distant satellite galaxies in the Local Group. The group of methods linking classical satellite galaxies in our MW to simulated subhalos is described in Sec. 8. Finally, we briefly mention a non-dynamical measurement in Sec. 9. We summarize these methods and discuss the role of modern numerical simulations in Sec. 10.

The readers will see that almost all methods have to assume a realistic potential model at the first place. Methods

1) Large Sky Area Multi-Object Fiber Spectroscopic Telescope 
from Sec. 2 to Sec. 6 mainly stem on the framework of modeling the observed positions and velocities (or phase-space distribution) of tracers. Many of the measurements described in Sec. 7 and Sec. 8 rely on calibrations made through modern numerical simulations of MW-like galaxies in a cosmological context.

Throughout the paper, unless otherwise stated, we quote the enclosed mass within a given radius and the $M_{200}$ virial mass, with 1- $\sigma$ errors. Different virial mass definitions are converted to $M_{200}$ assuming the NFW model profile, and different percentage of confidence regions are converted to 1- $\sigma$ errors assuming the errors are Gaussian in linear space if the upper and lower errors have comparable sizes, or are Gaussian in log space if the upper and lower errors are more comparable in log space.

\section{Galactic escape velocities: high and hyperve- locity objects}

The Galactic escape velocity is a fundamental quantity reflecting the depth of the underlying potential for our MW. It can be constrained using a variety of tracers, such as the high velocity stars in the tail of the velocity distribution for the population of halo stars, hypervelocity stars which are believed to be ejected from the Galactic center, and a few satellite galaxies moving with high velocities. In the following we introduce those fast moving objects and the approaches to model them. Measurements in this section fall in the categories of "Escape V HVS" and "Leo I" in Fig. 1.

\subsection{The high velocity tail distribution of halo stars}

Early attempts of measuring the Galactic escape velocity can be traced back to the 1920 s and 1960s [e.g. 288, 289]. The measurements were based on modeling the observed high velocity stars with an analytical functional form describing the velocity distribution of these stars near the high velocity tail. The readers can find more details about the full phasespace distribution function of dynamical tracer objects within a given potential in Sec. 5. Here we only briefly introduce the idea. The Jeans theorem states that the distribution of tracers in a dynamical system can be described by integrals of motion. The asymptotic form of the distribution function near the high velocity tail can be approximated as a power law

$f(E) \propto E^{k}$,

where the energy $E$ is defined through $E=-\Phi-v^{2} / 2$, with $\Phi$ and $v^{2} / 2$ being the potential and kinematic energy. $k$ de- scribes the shape of the distribution at the high velocity end. The potential energy is defined such that $\Phi\left(r_{\max }\right)=0$ at some maximum radius, $r_{\max }$, of the Galaxy, beyond which the star is considered to have "escaped". Under such a definition, the escape velocity is simply given by

$\Phi(r)=-\frac{1}{2} v_{\mathrm{esc}}^{2}(r)$.

Thus

$f\left(v \mid v_{\mathrm{esc}}, k\right) \propto\left(v_{\mathrm{esc}}^{2}-v^{2}\right)^{k} \quad\left(\right.$ for $\left.v<v_{\mathrm{esc}}\right)$,

where $v=|\mathbf{v}|$.

In 1990, Leonard and Tremaine [231] suggested that the term $\left(v_{\text {esc }}+v\right)^{k}$ can be dropped and the velocity distribution of stars at the high velocity end can be modeled by the following formula

$f\left(v \mid v_{\mathrm{esc}}, k\right) \propto\left(v_{\mathrm{esc}}-v\right)^{k} \quad\left(v<v_{\mathrm{esc}}\right)$.

Integrating Eqn. 3 or Eqn. 4 over tangential velocities, the radial velocity distribution is

$f\left(v_{r} \mid v_{\mathrm{esc}}, k\right)=\int f\left(v \mid v_{\mathrm{esc}}, k\right) \delta\left(v_{r}-\mathbf{v} \cdot \mathbf{n}\right) \mathrm{d}^{3} v$,

where $\mathbf{n}$ is a unit vector along the line of sight.

Basically, spectroscopic observations can be used to measure line-of-sight velocities with respect to the Sun. If we know the solar motion ${ }^{2)}$, Heliocentric distances and velocities can be used to obtain radial velocities with respect to the Galactic center. When proper motions are not available and hence tangential velocities are difficult to be robustly inferred $^{3)}$, Eqn. 4 can be used to compare with the measured radial velocities of high velocity stars, and constrain the escape velocity, $v_{\mathrm{esc}}$, at the Galactocentric radius, $r$, of the star. Besides, the measurement errors of line-of-sight velocities were typically much smaller than the uncertainties of tangential velocities inferred from proper motions. Based on simulated data, Leonard and Tremaine in 1990 [231] showed that estimates made using only radial velocities were as accurate as those made when employing proper motion data with large uncertainties.

Using Eqn. 4, the local escape velocity at the solar neighborhood was estimated to be in the range of 450 to $650 \mathrm{~km} / \mathrm{s}$ (90\% confidence level) by Leonard and Tremaine in 1990 [231]. A subsequent work by Kochanek in 1996 [213] adopted Eqn. 3 and refined the escape velocity to be in the range of $489 \mathrm{~km} / \mathrm{s}$ to $730 \mathrm{~km} / \mathrm{s}$ ( $90 \%$ confidence level). These early studies were limited by the small sample size of available high velocity stars. More recently, with continuously

2) Details about how to measure the solar motion are provided in Sec. 3

3) By fitting analytical velocity-space or phase-space distributions to observed line-of-sight velocities, it is still possible to model and infer the tangential velocity components (see more details in Sec. 4.3) 
growing spectroscopic observations, the sample of high velocity stars with available radial velocity measurements had significantly increased.

Based on high velocity stars selected from an early internal data release of the RAVE survey, Smith et al. in 2007 [357] modeled their radial velocity distributions following Eqn. 3. Cosmological simulations of disk galaxies were used in their study to determine the limit for the parameter $k$. The local escape velocity was estimated to be $544_{-46}^{+64} \mathrm{~km} / \mathrm{s}$ (90\% confidence), and the mass within $50 \mathrm{kpc}$ was found to be in the range of 3.6 to $4.0 \times 10^{11} \mathrm{M}_{\odot}$. Adopting an adiabatically contracted NFW halo model, the virial mass of our MW was estimated to be $M_{200}=1.42_{-0.36}^{+0.49} \times 10^{12} \mathrm{M}_{\odot}$.

With increased sample of stars from the fourth data release (DR4) of RAVE, Piffl et al. in 2014 [312] repeated the modeling using Eqn. 4, and used hydrodynamical simulations to validate the functional form, set a prior for the parameter $k$, and choose a minimum velocity cut for stars and the $r_{\text {max }}$ escape radius. Because the increased sample of stars covered a broader distance range than those in previous studies, the position information of stars was also incorporated into their modeling. This was achieved by either scaling the escape velocity at different distances to the solar position through Eqn. 2, or by analyzing stars at different distances separately. The local escape velocity was updated to be $533_{-41}^{+54} \mathrm{~km} / \mathrm{s}$ (90\% confidence). Assuming an NFW profile for the dark matter halo, the virial mass was estimated to be $M_{200}=1.60_{-0.25}^{+0.29} \times 10^{12} \mathrm{M}_{\odot}$, which is in a good statistical agreement with the earlier study of Smith et al. in 2007 [357].

The sample of stars used by Smith et al. in 2007 and Piffl et al. in 2014 was rather small. In 2017, Williams et al. [401] selected intrinsically bright main sequence turn off (hereafter MSTO) stars, blue horizontal branch (hereafter BHB) stars and K-giants with measured distances and line-of-sight velocities from SDSS/DR9, among which 2000 stars are above their minimum velocity cut as high velocity halo stars. Their sample of high velocity stars spans $\sim 40 \mathrm{kpc}$ in distance, from the solar vicinity to $\sim 50 \mathrm{kpc}$. [401] considered in their Bayesian modeling the radial dependence of the escape velocity, the distance errors and possible contamination by outliers. The local escape velocity was constrained to be $521_{-30}^{+46} \mathrm{~km} / \mathrm{s}$, and the escape velocity drops to $379_{-28}^{+34} \mathrm{~km} / \mathrm{s}$ at $50 \mathrm{kpc}$ (94\% confidence region). The prior for the parameter $k$ was allowed to be flat over a much broader region given their larger sample of stars, which served to directly constrain the values of $k$ from data. $k$ does not seem to be a strong function of positions. For MSTO and K-giants, $k$ was approximately constrained to be $4 \pm 1$, while the value for BHBs was slightly favored to be higher. Given Eqn. 2 and $M(<r)=\frac{r^{2}}{G} \frac{\mathrm{d} \Phi}{\mathrm{d} r}$. The escape velocity measured by [401] over 6 and $50 \mathrm{kpc}$ can be converted to the enclosed mass or rotation velocity as a function of distance. The mass within $50 \mathrm{kpc}$ was best constrained to be $2.98_{-0.52}^{+0.69} \times 10^{11} \mathrm{M}_{\odot}$.

The launch of Gaia had led to a significant increase in the sample of high velocity stars within a few kpc from the Sun. Based on Gaia DR2, Monari et al. in 2018 [265] selected a sample of 2,850 counter-rotating halo stars, to be distinguished from stars in the MW disk. They measured the escape velocity curve between $5 \mathrm{kpc}$ and $10.5 \mathrm{kpc}$, and the local escape velocity was updated to be $580 \pm 63 \mathrm{~km} / \mathrm{s}$. Adopting an NFW profile plus a disk and a bulge component given by Irrgang et al. [191], the virial mass of our MW was estimated to be $M_{200}=1.28_{-0.50}^{+0.68} \times 10^{12} \mathrm{M}_{\odot}$.

Very recently in 2019, Deason et al. [96] selected $\sim 2,300$ counter-rotating halo stars, out of which $\sim 240$ have total velocities larger than $300 \mathrm{~km} / \mathrm{s}$, and are between Galactocentric distances of 4 and $12 \mathrm{kpc}$. Deason et al. [96] adopted both analytical distributions and the AURIGA simulations [158] to investigate the dependence of the parameter $k$ on various properties, including the velocity anisotropies (see Sec. 4 for more details about the definition of velocity anisotropy) and number density profiles of stars. The recent discovery of the "Gaia Sausage" structure [e.g. 14] in our MW, which was due to the merger of a dwarf galaxy and shows that halo stars in the solar vicinity have strong radially biased velocity anisotropy, helps to set a prior of $1<k<2.5$. This is smaller than those adopted by Monari et al. [265] and Piffl et al. [312]. The escape velocity at solar radius was estimated by Deason et al. [96] to be $528_{-25}^{+24} \mathrm{~km} / \mathrm{s}$. Assuming NFW profiles, the virial mass was best constrained to be $M_{200}=1.00_{-0.24}^{+0.31} \times 10^{12} \mathrm{M}_{\odot}$.

In a follow-up study by Grand et al. in 2019 [157], the effects of substructures were visited by applying the approach of Deason et al. [96], with slight modifications, to the set of AURIGA simulations [158]. The recovered virial masses had a median falling $\sim 20 \%$ below the true values, with a scatter of roughly a factor of 2 . After correcting for the bias, the MW virial mass was revised as $M_{200}=1.29_{-0.47}^{+0.37} \times 10^{12} \mathrm{M}_{\odot}$, with extra systematic uncertainties to be kept in mind.

\subsection{Bound and unbound hypervelocity stars}

Unbound hypervelocity stars exceed the Galactic escape velocity and are usually believed to form through exotic mechanisms such as ejections by the super massive black hole in the Galactic center. Such hypervelocity stars have been detected in the outer stellar halo (see the review paper by Brown et al. in 2015 [60]). Due to the strategy and instruments used for detection, many previously detected hypervelocity stars are early-type stars.

It was found that the trajectories of these early-type hypervelocity stars are consistent with coming from the Galactic 
center. Thus they are very likely formed through the gravitational interaction with the super massive black hole ( $\operatorname{Sgr} \mathrm{A}^{*}$ ) in the Galactic center and as a result gained such high velocities [e.g. 73, 133, 151, 152, 287, 417]. More specifically, observations are consistent with a scenario that each of the hypervelocity stars originally belonged to a binary star system, and the system was tidally dissociated by Sgr A*. The process is called the "Hills" mechanism [183]. This mechanism is demonstrated in Fig. 3. One member of the binary system got ejected, while the other stayed in the Galactic center. In particular, this picture is consistent with the observational fact that within $r \sim 0.5 \mathrm{kpc}$ to the Galactic center, young stars have been observed [e.g. 193, 244, 277, 295], which otherwise might challenge our knowledge of star formation because molecular clouds are difficult to survive strong tidal forces in the Galactic center.

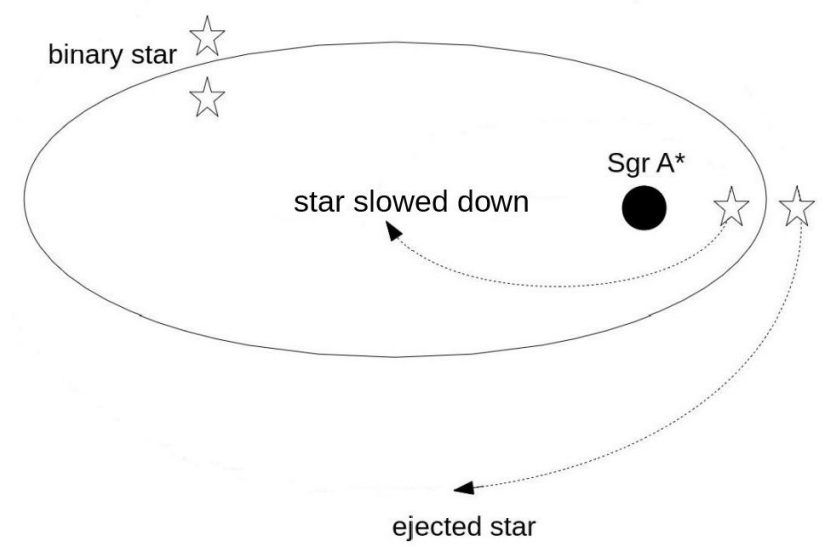

Figure 3 Demonstration of the Hills mechanism. The binary star system is dissolved by the super massive black in the Galactic center, Sgr A*. One member star of the binary system gets slowed down and stays close to the Galactic center of our MW. According to energy conservation, the other star gains very high velocity and gets ejected. This plot referenced Fig. 2 of [60].

A similar sample of bound hypervelocity stars, which are likely stars ejected from the Galactic center through the same mechanism [59], but whose velocities are still below the escape velocity, have been observed as well [62, 63, 64].

There are alternative models for the formation of hypervelocity stars. For example, they could be ejected by the Large Magellanic Cloud or be runaways from the MW disk [38, 39, 123]. Despite these debates, if the early-type hypervelocity stars indeed form through the ejection by Sgr A*, they not only contain information about processes happened in the Galactic center, but can also be used to constrain the shape [e.g. 154, 416] and depth [e.g. 134, 300, 323, 343] of the potential.

Assuming the Sgr A* ejection scenario is true, Rossi et al. [323] modeled the velocity distribution of observed hypervelocity stars in their 2017 study, following the model in a series of earlier papers [212, 322].

If there is only the black hole potential, the velocity for the ejected star at infinity is given by

$v_{\text {eject }}=\sqrt{\frac{2 G m_{2}}{a}}\left(\frac{M}{m_{t}}\right)^{1 / 6}$,

where $M$ is the central black hole mass, $m_{t}$ is the total mass of the binary, $a$ is the binary separation and $m_{2}$ is the mass of the companion star in the binary system [336].

Rossi et al. [323] modeled the distribution of binary separations and mass ratios as power-law forms, which then predicted the distribution of ejecting velocities through Eqn. 6. After ejection, the change in velocity of each star can be either calculated by assuming some escape velocity out to $50 \mathrm{kpc}$ or be calculated by adopting some model potential including components of the Galactic disk, bulge and dark matter halo. Assuming the ejection rate and life time of stars, the predicted velocity distribution of these stars can be compared with the true velocities of observed hypervelocity stars, and thus constrain the adopted escape velocity or potential models. Their analysis favored halos with escape velocity from the Galactic center to $50 \mathrm{kpc}$ smaller than $850 \mathrm{~km} / \mathrm{s}$, which then favored $\Lambda \mathrm{CDM}$ halos with $M_{200}$ in the range of 0.5 and $1.5 \times 10^{12} \mathrm{M}_{\odot}$.

Perets et al. [300] proposed an independent method of using the asymmetric distribution for ingoing and outgoing hypervelocity stars to constrain the MW potential in 2009. Ejected stars exceeding the escape velocity are unbound and will leave our MW, whereas bound ejected stars will reach the apocenter and turn back. Thus bound stars can contribute to both ingoing stars with negative velocities [58, 60, 206] and outgoing stars with positive velocities. Unbound stars only contribute to the outgoing population, which introduces an asymmetry in the high velocity tail of the velocity distribution. Indeed, such asymmetry has been observed, with a significant excess of stars traveling with radial velocities larger than $275 \mathrm{~km} / \mathrm{s}$ [63].

The asymmetry is also related to the lifetime of such ejected stars. Some stars might have evolved to a different stage before reaching to a large enough Galactocentric distance and turning back. Note they do not totally disappear, but may have evolved out of the detection range of corresponding instruments. For example, the MMT (Multiple Mirror Telescope) hypervelocity star survey [61, 62] mostly target the main sequence B stars. Fragione and Loeb in 2017 [134] modeled the observed asymmetry by varying both the potential model and the life or travel time of hypervelocity stars. If fixing the travel time of hypervelocity stars to 330 Myr for typical B stars, the MW virial mass $M_{200}$ was found to be in the range of 1.2 to $1.9 \times 10^{12} \mathrm{M}_{\odot}$. 
More recently, with Gaia DR2, previously identified hypervelocity stars with only line-of-sight velocities were revisited and extended to have full 6-dimensional phase-space information based on Gaia proper motions [e.g. 40, 65, 190]. More hypervelocity star candidates, especially late-type stars 4), have been reported and predicted [e.g. 176, 252, 253]. Gaia proper motions enabled further and more robust investigations on the origin for hypervelocity star candidates.

While those previously discovered early-type hypervelocity stars are very likely from the Galactic center, the origin of late-type hypervelocity stars is not clear. Based on proper motions and radial velocities, Boubert et al. in 2018 [40] concluded that in fact almost all previously-known late-type hypervelocity stars are very likely bound to our Milky Way. A similar conclusion wasreached by Hawkins and Wyse in 2018 [177] based on chemical abundance patterns, that a few candidate hypervelocity stars are most likely bound high velocity halo stars, which are close to the high velocity tail of the distribution, but are unlikely hypervelocity stars ejected from the Galactic center or from the Large Magellanic Cloud.

Hattori et al. in 2018 [176] discovered 30 stars with extreme velocities ( $\geqslant 480 \mathrm{~km} / \mathrm{s}$ ) from Gaia DR2. Tracing their orbits, they reported that at least one of the stars is consistent with having been ejected from the Galactic center. Unlike previous observations of early-type hypervelocity stars, these stars are quite old, with chemical properties similar to the Galactic halo. Assuming these stars are bound, the virial mass of our MW should be higher than $1.4 \times 10^{12} \mathrm{M}_{\odot}$.

\subsection{Bound and unbound satellite galaxies}

Other types of fast moving objects such as dwarf satellite galaxies can be used to constrain the mass of our MW as well. Among the MW satellite galaxies, Leo I plays an important role since it has a large Galactocentric distance and a high velocity [e.g. 361], which could suggest that Leo I is only weakly bound (if at all) to the MW. Incorporating Leo I into the analysis has to rely on the assumption that Leo I is bound to our MW. As a result, a heavy MW is often required to keep Leo I bound given its large distance and high velocity (see more details in Sec. 4.4, Sec. 5.1 and Sec. 7). Based on subhalos in MW-like halos from the Aquarius simulation [365], Boylan-Kolchin et al. [55] demonstrated in 2013 that Leo I is very unlikely to be unbound, because $99.9 \%$ subhalos in their simulations are bound to their host halos. To keep Leo I bound, Boylan-Kolchin et al. [55] estimated the virial mass of our MW to be $M_{200}=1.34_{-0.31}^{+0.41} \times 10^{12} \mathrm{M}_{\odot}$.
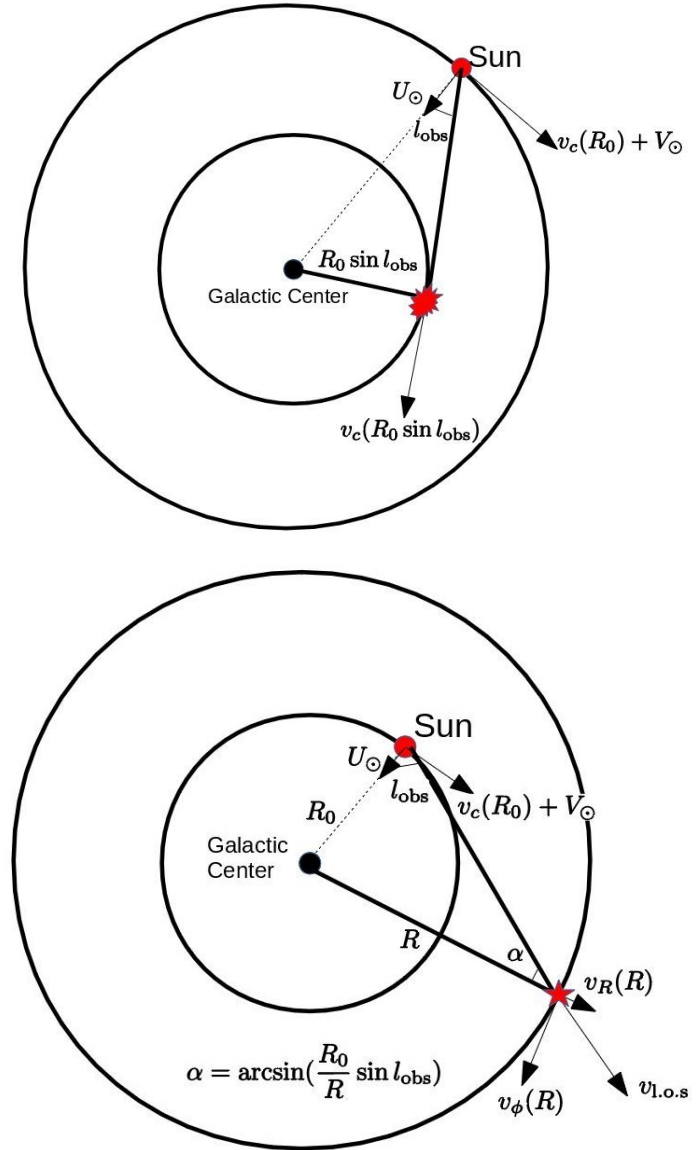

Figure 4 Top: Plot showing the concept of terminal velocity, for a gas cloud inside the Galactic disk and within the solar radius. The gas is moving along a circular orbit, and the maximum velocity which can be observed along that circular orbit happens at the tangent point with Galactic longitude of $l_{\mathrm{obs}} . R_{0}$ is the Galactocentric distance of our Sun, and $R_{0} \sin l_{\mathrm{obs}}$ is the Galactocentric distance for the gas cloud. $v_{c}\left(R_{0} \sin l_{\text {obs }}\right)$ is the circular velocity at the radius of the gas cloud. The terminal velocity is $v_{c}\left(R_{0} \sin l_{\mathrm{obs}}\right)$ minus the corresponding velocity components of the rotation velocity for the Local Standard of Rest $\left(v_{c}\left(R_{0}\right)\right)$ and the peculiar solar motion $\left(U_{\odot}\right.$ and $\left.V_{\odot}\right)$ with respect to the Local Standard of Rest, projected along the line of sight. Both $U_{\odot}$ and $V_{\odot}$ are in fact much smaller than $v_{c}\left(R_{0}\right)$. Bottom: Plot showing the concept of the line-of-sight velocity for a star within the Galactic disk and outside the solar radius. The star is assumed to be observed at Galactic longitude of $l_{\mathrm{obs}} . R$ and $R_{0}$ are the Galactocentric distance of the star and our Sun. $v_{R}(R)$ and $v_{\phi}(R)$ are the radial and tangential velocities of the star with respect to the Galactic center. $U_{\odot}$ and $V_{\odot}$ reflect the peculiar motion of our Sun, and $v_{c}\left(R_{0}\right)$ is the circular velocity of the Local Standard of Rest. They are the same as those defined in the top plot. Both $U_{\odot}$ and $V_{\odot}$ are in fact much smaller than $v_{c}\left(R_{0}\right) . v_{R}(R)$ is much smaller than $v_{\phi}(R)$. The line-ofsight velocity of the star with respect to us is the velocity difference between the star and our Sun projected along the line-of-sight direction.

Using Gaia DR2 proper motion data, member stars of a few MW satellite galaxies can be more robustly identified, which then provide the averaged proper motions of these satellite galaxies. Fritz et al. in 2018 [136] derived proper motions for 39 companion galaxies of our MW out to 420

4) Stars whose spectral types are F, G, K or M, including both dwarf and giant stars. 
kpc. Based on arguments of keeping acceptable distributions of orbital apocenters and having a reasonable fraction of bound satellites, they reported that a heavy MW ( $1.6 \times$ $\left.10^{12} \mathrm{M}_{\odot}\right)$ is more preferable than a light MW $\left(0.8 \times 10^{12} \mathrm{M}_{\odot}\right)$.

\section{Rotation velocities of the inner MW: local observables}

The Galactic rotation curve (circular or rotation velocity as a function of radius) can directly reflect the mass enclosed within different radii. In this section, we introduce local observables and corresponding methods of measuring the rotation velocities of the inner MW. Measurements in this section fall in the category of "LocalObs rot V" in Fig. 1.

To measure the shape of the rotation curve within the solar orbit, previous studies have typically employed the terminal velocities of the interstellar medium (ISM) or HI gas clouds [e.g. 168, 232, 359, 377]. The basic idea relies on the fact that for circular orbits in an axis-symmetric potential and within the solar orbital radius, the observed peak velocity of ISM along any line of sight in the Galactic disk plane corresponds to the gas at the tangent point. The approximation of circular orbits is reasonable given the fact that the inner region of our MW is dominated by the disk component. In other words, the terminal velocity tells that there is a particular direction, along which the rotation velocity of circular orbits entirely contributes to the line-of-sight velocity. This is demonstrated in the top plot of Fig. 4.

We use $R_{0}$ to represent the Galactocentric distance of our Sun, and we assume the peak velocity is observed at Galactic latitude of $b=0$ (in the Galactic disk plane) and Galactic longitude of $l=l_{\mathrm{obs}}$. The Galactocentric distance of the observed IGM is $R=R_{0} \sin l_{\text {obs }}$, and the terminal velocity at $R$ [262] is

$$
\begin{aligned}
v_{\text {terminal }}\left(R_{0} \sin l_{\mathrm{obs}}\right) & =v_{c}\left(R_{0} \sin l_{\mathrm{obs}}\right) \\
& -\left(v_{c}\left(R_{0}\right)+V_{\odot}\right) \sin l_{\mathrm{obs}}-U_{\odot} \cos l_{\mathrm{obs}} .
\end{aligned}
$$

$v_{c}\left(R_{0} \sin l_{\mathrm{obs}}\right)$ and $v_{c}\left(R_{0}\right)$ are the rotation velocities at $R=$ $R_{0} \sin l_{\text {obs }}$ for the observed IGM and at $R_{0}$ for our Sun, respectively. The second term refers to the rotation of Local Standard of Rest (hereafter LSR), $v_{c}\left(R_{0}\right)$, and the motion of our Sun with respect to the LSR in the direction of Galactic rotation, $V_{\odot}$. Note the LSR follows the mean motion of material in the neighborhood of the Sun, which is often assumed to be circular, and the Sun has a small peculiar motion relative to the LSR. $U_{\odot}$ is the velocity towards Galactic center. The solar motion with respect to the Galactic center is a combination of the velocity of the LSR and the peculiar motion of the Sun with respect to the LSR in the same direction

$$
\mathbf{V}_{\odot}=\left(U_{\odot}, v_{c}\left(R_{0}\right)+V_{\odot}, W_{\odot}\right),
$$

where $W_{\odot}$ is the velocity component of the solar peculiar motion perpendicular to the Galactic disk. Note the velocity components for solar peculiar motion, $U_{\odot}, V_{\odot}$ and $W_{\odot}$ are all much smaller than the rotation velocity of the $\operatorname{LSR}, v_{c}\left(R_{0}\right)$.

Assuming the peculiar motions of the Sun, $U_{\odot}, V_{\odot}$ and $W_{\odot}$, are well determined, which we will discuss later, terms of $U_{\odot}$ and $V_{\odot}$ can be moved to the left side as known quantities. The right hand side of Eqn. 7 becomes $v_{c}\left(R_{0} \sin l_{\text {obs }}\right)-$ $v_{c}\left(R_{0}\right) \sin l_{\mathrm{obs}}$, which can be reduced to $\frac{v_{c}\left(R_{0} \sin l_{\mathrm{obs}}\right)}{R_{0} \sin l_{\mathrm{obs}}}-\frac{v_{c}\left(R_{0}\right)}{R_{0}}$ after divided by $R_{0} \sin l_{\text {obs }}$ [e.g. 99]. Hence given the observed terminal velocities, plus the Galactocentric distance of our Sun and the solar motion, the shape of the rotation curve can be determined.

The normalization of the rotation curve can be determined, by measuring the absolute rotation velocity for the LSR, $v_{c}\left(R_{0}\right)$, for example. We will discuss later in this section about how to measure $v_{c}\left(R_{0}\right)$.

Terminal velocities are usually adopted to measure the shape of the rotation curve within the orbit of our Sun. For regions slightly outside the Sun's Galactocentric radius but still on the Galactic disk, measurements of the rotation velocities can be made by modeling observed distances and lineof-sight velocities of maser sources and disk stars [e.g. 135]. In particular, astrophysical maser sources are associated with high-mass star forming regions, which are expected to be on nearly circular orbits in the Galactic disk. Because the emission of masers is a narrow spectral line, the Heliocentric parallaxes, proper motions and line-of-sight velocities of maser sources can be very well measured based on radio interferometry.

This is demonstrated in the bottom plot of Fig. 4. The observed line-of-sight velocity, $v_{\text {l.o.s }}$, of a maser source or disk star outside the solar radius at Galactic latitude $b=0$, Galactic longitude $l=l_{\text {obs }}$ and Galactocentric distance $R$ is given by

$$
\begin{array}{r}
v_{\text {l.o.s }}=v_{\phi}(R) \sin \left(\arcsin \left(\frac{R_{0}}{R} \sin l_{\mathrm{obs}}\right)\right) \\
-\left(v_{c}\left(R_{0}\right)+V_{\odot}\right) \sin \left(l_{\mathrm{obs}}\right) \\
+v_{R}(R) \cos \left(\arcsin \left(\frac{R_{0}}{R} \sin l_{\mathrm{obs}}\right)\right) \\
-U_{\odot} \cos \left(l_{\mathrm{obs}}\right),
\end{array}
$$

where $U_{\odot}, V_{\odot}$ and $R_{0}$ are still the peculiar solar motions towards Galactic center and in the direction of Galactic rotation, and the Galactocentric distance of our Sun. $v_{c}\left(R_{0}\right)$ is the rotation velocity of the LSR. $v_{\phi}(R)=v_{c}(R)-v_{a}(R)$ is the tangential velocity component of the maser source or disk star at $R . \quad v_{c}(R)$ is the rotation velocity at $R$, and $v_{a}(R)$ is introduced to describe the asymmetric drift by Binney and Tremaine (2008). $v_{R}(R)$ is the radial motion of the observed 
maser source or star, which is much smaller than $v_{c}(R)$ or $v_{\phi}(R)$.

Similarly, once we know the solar motion and its Galactocentric distance, the rotation velocity of the LSR and the asymmetric drift term, we can constrain the rotation velocity $v_{c}(R)$ for the source observed at $R$ through Eqn. 9. The radial motion, $v_{R}(R)$, can be treated as a free parameter or averaged to zero over a sample of sources.

We now briefly introduce how to measure the rotation velocity of the LSR and the peculiar motion of our Sun. These can be inferred through the apparent motion of Sgr A* in the Galactic Center [e.g. 149, 318, 339]. If Sgr A* is at rest in the Galactic frame, the apparent motion of Sgr A* reflects the absolute motion of our Sun with respect to the Galactic center. The peculiar motion of our Sun can then be decoupled from the rotation velocity of the LSR through the observation of kinematics from nearby stars. In addition, the accurate Heliocentric distances and line-of-sight velocities of maser sources can be used to jointly model and constrain the rotation velocities of masers themselves with respect to the Galactic center, the rotation velocity of the LSR, the Galactocentric distance and the peculiar motion of our Sun [e.g. 47, 66, 188, 263, 319].

While the terminal velocities within the solar radius and the line-of-sight velocities of sources slightly outside the solar radius but within the Galactic disk are traditional observables to constrain the rotation velocities for the inner MW, it is necessary to mention that in 2012, Bovy et al. [44] was probably the first to use hot stellar tracers out of the MW disk to measure the MW rotation curve between $4 \mathrm{kpc}$ and $14 \mathrm{kpc}$, based on the spherical Jeans equation and phase-space distribution functions. More recently in 2018, with spectroscopic data from APOGEE and photometric data from WISE, 2MASS and Gaia to get precise parallaxes and hence full sixdimensional phase-space coordinates, Eilers et al. [117] measured the rotation velocity curve based on the Jeans equation with an axisymmetric potential from $5 \mathrm{kpc}$ to $25 \mathrm{kpc}$ to the Galactic center. We briefly mention their efforts here, and postpone discussions about the (spherical) Jeans equation and the distribution function to Sec. 4 and Sec. 5. The readers can also check Sec. 6 about constraining the local rotation velocity using the GD-1 stream [217, 251].

Started from the last century, numerous efforts had been spent to constrain potential models for the Galactic disk, bulge and the dark matter halo using the measured circular velocities of the inner MW. These studies were often combined with a few other observables for the inner MW (typically based on stellar dynamics or star counting) in the fol- lowing.

- The local vertical force some distance above the Galactic disk or the total surface density within a cylinder crossing the disk [e.g. 49, 185, 219, 427], measured with the observed distances and radial velocities of stars in the Galactic pole and the vertical Jeans equation.

- Total local volume density [e.g. 184], measured with stars in the solar vicinity

- Local surface density of visible matter in the disk [e.g. $185,218]$

- The velocity dispersion in Baade's window ${ }^{5)}$ to the Galactic center [e.g. 320].

- The mass in the very central parsec regions.

In addition, as the readers will see, in order to constrain the mass of our MW out to large distances, the above local observables are not enough, and measurements made by other alternative methods based on more distant tracer objects should be adopted.

Early attempts of this kind can be traced back to 1998 [e.g. 99], when Dehnen and Binney jointly modeled the observed terminal velocities, distances and line-of-sight velocities of maser sources, local vertical forces, surface densities and the observed velocity dispersion of the bulge in Baades window. Combined with other contemporary measurements of the enclosed mass within $100 \mathrm{kpc}$ to the Galactic center by Kochanek [213], which was mainly based on phase-space distribution functions (see Sec. 5 for more details), the rotation curves out to $100 \mathrm{kpc}$ were obtained for different models. The total mass within $100 \mathrm{kpc}$ was constrained to be in the range of $3.41 \times 10^{11} \mathrm{M}_{\odot}$ and $6.95 \times 10^{11} \mathrm{M}_{\odot}$.

In 2002, Klypin et al. [210] presented a set of gravitational potential models for our MW, based on standard disk formation theory and adiabatic compression of baryons within cuspy dark matter halos. Models with and without the exchange of angular momentum between baryons and dark matter were both considered. The models with a range of different parameters were tested against the terminal velocities, the circular velocities slightly outside the solar radius, the local surface density of gas and stars, the vertical force at $1.1 \mathrm{kpc}$ above the Galactic disk and the mass in the very central parsec regions of our MW. Klypin et al. [210] also included the enclosed mass within $100 \mathrm{kpc}$ to the Galactic center from other studies [99, 213], measured with distribution functions, and found that their modeling preferred our MW to have virial mass of about $M_{200}=0.86 \times 10^{12} \mathrm{M}_{\odot}$, though their analysis was not based on strict statistical inferences.

5) A sky area with relatively low amounts of interstellar dust along the line of sight, which is an observational window to the obscured Galactic Center of the MW. 
Weber and Boer in 2010 [396] constrained the local dark matter density. They made best fit to observed data including the total mass within the solar orbital radius, the total density and the surface density of visible matter at the solar position, the local vertical force, the shape of the rotation curve within the Galactic disk [359], and the constrained mass of our MW within given radii from other studies [10, 400, 411]. Given the correlations among local dark matter density, the scale length of the dark matter halo and the Galactic disk, and since the scale lengths were poorly constrained, their best-fit local dark matter density varied from 0.005 to $0.01 \mathrm{M}_{\odot} \mathrm{pc}^{-3}$, which allowed the total mass of our MW up to $2 \times 10^{12} \mathrm{M}_{\odot}$.

More recently in 2011, McMillan [261] jointly modeled the observed terminal velocities, maser sources and the local vertical force using their disk, bulge plus dark halo models. However, for the mass enclosed within even larger distances, McMillan still had to refer to other contemporary measurements based on the distribution function [400]. Their best-fit rotation curve extended to $\sim 100 \mathrm{kpc}$. The MW virial mass was measured to be $M_{200}=1.26 \pm 0.24 \times 10^{12} \mathrm{M}_{\odot}$. Later on, with more available maser observations, the measured virial mass was updated to be $M_{200}=1.3 \pm 0.3 \times 10^{12} \mathrm{M}_{\odot}$ in a follow-up paper [262].

In 2013, Irrgang et al. [191] adopted three different model potentials with disk, bulge and dark matter halo to constrain the mass of our MW within 50, 100 and $200 \mathrm{kpc}$. They jointly modeled the solar motion, the terminal velocities, the observations of maser sources, the local total mass density and the local surface mass density of the Galactic disk. The velocity dispersion in the bulge was used to constrain the inner most region, and an hypervelocity halo BHB star was assumed to be bound to our MW and hence put further constraints on the potential out to $200 \mathrm{kpc}$. The mass enclosed within $200 \mathrm{kpc}$ was constrained to be $1.9_{-0.8}^{+2.4} \times 10^{12} \mathrm{M}_{\odot}, 1.2_{-0.2}^{+0.1} \times 10^{12} \mathrm{M}_{\odot}$ and $3.0_{-1.1}^{+1.2} \times 10^{12} \mathrm{M}_{\odot}(90 \%$ confidence) for the three potential models adopted in their analysis, respectively.

Nesti and Salucci in 2013 [279] included in their analysis the observed velocity dispersion of halo stars out to $80 \mathrm{kpc}$, and used the spherical Jeans equation (see Sec. 4 for details) to obtain the rotation velocities out to such distances. They adopted both the Burkert (core) and NFW (cusp) profiles for the modeling, and the best constrained masses within $50 \mathrm{kpc}$ were $4.5_{-2.0}^{+3.5} \times 10^{11} \mathrm{M}_{\odot}$ and $4.8_{-1.5}^{+2.0} \times 10^{11} \mathrm{M}_{\odot}$ for the Burkert and NFW model profiles, respectively. The masses within $100 \mathrm{kpc}$ were $6.7_{-3.3}^{+6.7} \times 10^{11} \mathrm{M}_{\odot}$ and $8.1_{-3.2}^{+6.0} \times 10^{11} \mathrm{M}_{\odot}$, respectively. The virial masses of the best-fit Burkert and NFW profiles were extrapolated to be $1.11_{-0.61}^{+1.6} \times 10^{12} \mathrm{M}_{\odot}$ and
$1.53_{-0.77}^{+2.3} \times 10^{12} \mathrm{M}_{\odot}{ }^{6)}$.

The galpy software, which is a python package for galactic-dynamics calculations, was developed by Bovy in 2015 [42]. It incorporated an example potential model with disk, bulge and halo components. The model potential was based on fits to local observables including the terminal velocities, the velocity dispersion through the Baade's window, the local vertical force, the local visible surface density and the local total density, in combination with the rotation curve measured by Bovy et al. in 2012 [44] at the solar neighborhood (see above) and the total mass within $60 \mathrm{kpc}$ to the Galactic center of [411] through the spherical Jeans equation (see Sec. 4 for details). Their model potential preferred a virial mass of about $M_{200}=0.7 \times 10^{12} \mathrm{M}_{\odot}$.

In two subsequent papers, Bajkova and Bobylev in 2016 $[6,32]$ used the spherical Jeans equation to fit a bulge and a disk together with a few different halo models to the lineof-sight velocities of hydrogen clouds at the tangent points, kinematic and parallax data of 130 maser sources within $25 \mathrm{kpc}$, as well as more distant rotation velocity measurements by [23]. If adopting the NFW model profile for the halo, the mass within $200 \mathrm{kpc}$ was constrained to be $7.5 \pm 1.9 \times 10^{11} \mathrm{M}_{\odot}$.

Recently, Cautun et al. [76] in 2019 have combined the stellar rotation curve measured by Gaia [117] with the outer mass measurements from satellite dynamics [72] to constrain both the stellar and the dark matter distribution of the MW. They have used a contracted dark matter halo model with free mass and concentration, and stellar bulge and disk components with several free parameters. Their best-fit model corresponds to a total MW mass, $M_{200}=1.12_{-0.22}^{+0.20} \times 10^{12} \mathrm{M}_{\odot}$, and a dark matter halo concentration (before baryonic contraction), $c=8.2_{-1.5}^{+1.7}$, which is typical of a $10^{12} \mathrm{M}_{\odot}$ halo. Furthermore, Cautun et al. [76] have shown that the same data is equally well fit by an NFW halo profile, but with a 20 percent lower halo mass, much higher concentration and a 20 percent higher stellar mass. It illustrates that the rotation curve for distances below $20 \mathrm{kpc}$ cannot break the degeneracy between the halo and the stellar mass profiles, and thus, because the MW baryonic profile is still poorly understood, the inferred halo mass depends on the baryonic model employed in a given study.

Combining observations of rotation velocities for the inner MW compiled by $[189,294]$ and the rotation velocities up to $100 \mathrm{kpc}$ obtained through the Spherical Jeans Equation by [189], Karukes et al. in 2019 constrained the virial mass of our MW to be $M_{200}=0.89_{-0.08}^{+0.10} \times 10^{12} \mathrm{M}_{\odot}$.

6) The errors are $95.45 \%$ confidence level $(2-\sigma)$. Through private communications with the authors, the main driving uncertainty in the errors was the velocity anisotropy $\beta$, for which no prior can be known. So the errors cannot be simply converted to $1-\sigma$ uncertainties assuming Gaussian errors. The associated error in Fig. 1 is simply the $2-\sigma$ errors decreased by $10 \%$. 
Almost all the above studies had to rely on observations of more distant luminous objects and other alternative methods to infer the mass distribution out to larger distances, such as the spherical Jeans equation and the distribution function. We now move on to introduce how the mass of our MW can be constrained through the spherical Jeans equation, through the phase-space distribution function of dynamical tracer objects and through dynamical modeling of tidal streams in the following three sections (Sec. 4, Sec. 5 and Sec. 6).

\section{The rotation velocity out to large distances from the Jeans Equation: halo stars, globular clusters and satellite galaxies}

In the previous section, we introduced how the rotation curve of the inner MW can be inferred through the terminal and circular velocities. To obtain the rotation curve out to large distances, the spherical Jeans Equation has been frequently used. In the following, we start by introducing the spherical Jeans Equation and then move on to describe relevant studies in literature. Measurements in this section fall in the category of "SJE" in Fig. 1.

\subsection{The spherical Jeans Equation}

The dynamical structure of a system can be fully specified by its phase-space distribution function, or the number density of objects in phase space, $f(\mathbf{x}, \mathbf{v}, t) \equiv \mathrm{d}^{3} N / \mathrm{d}^{3} x \mathrm{~d}^{3} v$. In absence of collision, the phase-space density is conserved along the orbits of the particles, i.e., $\mathrm{d} f / \mathrm{d} t=0$, leading to the so-called collisionless Boltzmann equation

$\frac{\partial f}{\partial t}+\frac{\mathrm{d} \mathbf{v}}{\mathrm{d} t} \cdot \nabla_{\mathbf{v}} f+\mathbf{v} \cdot \nabla_{\mathbf{x}} f=0$

a manifestation of the Liouville theorem in classical mechanics. For a smooth distribution of particles, the particle acceleration is determined by the smooth potential field, $\mathrm{d} \mathbf{v} / \mathrm{d} t=-\nabla_{\mathbf{x}} \Phi$. Taking the first moment of the collisionless Boltzmann equation over velocity one can derive the more frequently used Jeans equation, which is a 6-dimensional analogy to the 3-dimensional Euler equations for fluid flow.

When the system is in a steady-state, both the underlying potential and the distribution function are independent of time, i.e., $\Phi(\mathbf{x}, t)=\Phi(\mathbf{x})$ and $\partial f / \partial t=0$. The Jeans equation then relates the potential gradients to observable quantities including the number density distribution, the mean velocity and velocity dispersions of different velocity components for observed objects.

Adopting the Jeans equation to constrain the potential gradient requires the knowledge of spatial derivatives of the velocity dispersions for different velocity components (e.g. the vertical, radial and azimuthal velocity dispersions and cross terms), which is not easy. Studies using the Jeans equation to constrain the underlying distribution of luminous and dark matter were traditionally limited to the solar neighborhood[e.g. 84, 184, 218], within a few kilo-parsecs from the Galactic plane [e.g. 50, 51, 145, 185, 219, 351, 358, 427] and out to about $\sim 10 \mathrm{kpc}$ with photometric distances [e.g. 241].

If further assuming the Galactic halo is spherical, we can derive the simplified and so far more frequently used spherical Jeans equation (hereafter SJE; Binney and Tremaine 1987):

$\frac{1}{\rho_{*}} \frac{\mathrm{d}\left(\rho_{*} \sigma_{r}^{2}\right)}{\mathrm{d} r}+\frac{2 \beta \sigma_{r}^{2}}{r}=-\frac{\mathrm{d} \Phi}{\mathrm{d} r}=-\frac{V_{c}^{2}}{r}$,

where quantities on the left side are the radial velocity dispersion of tracers in the system, $\sigma_{r}$, their radial density profile, $\rho_{*}$, and their velocity anisotropy, $\beta$. The velocity anisotropy is defined as

$\beta=1-\frac{\sigma_{\theta}^{2}+\sigma_{\phi}^{2}}{2 \sigma_{r}^{2}}=1-\frac{\left\langle v_{\theta}^{2}\right\rangle-\left\langle v_{\theta}\right\rangle^{2}+\left\langle v_{\phi}^{2}\right\rangle-\left\langle v_{\phi}\right\rangle^{2}}{2\left(\left\langle v_{r}^{2}\right\rangle-\left\langle v_{r}\right\rangle^{2}\right)}$,

where $\sigma_{\theta}$ and $\sigma_{\phi}$ are velocity dispersions of the two tangential components. $v_{r}$ is the radial velocity. $v_{\theta}$ and $v_{\phi}$ are the two components of the tangential velocity. When the quantities on the left-hand side of Eqn. 11 can be measured for observed luminous dynamical tracers, such as halo stars, globular clusters and satellite galaxies, the rotation velocity (or the potential gradient) on the right-hand side of the same equation can be directly inferred.

In reality, the observed quantity is the radial velocity dispersion of dynamical tracers, $\sigma_{r}$, converted from the Heliocentric line-of-sight velocities, and the tracer density profile, $\rho_{*}$. However, the velocity anisotropy, $\beta$, is more difficult to be properly measured if proper motions are not available, especially for tracer objects at large distances. It is obvious from Equation 11 that the velocity anisotropy term is degenerate with the gravity term, so that an overestimate of $\beta$ leads to an underestimate in mass. This is known as the mass-anisotropy degeneracy.

Assuming $\beta$ is constant, the solution to Equation 11 is

$\sigma_{r}^{2}(r)=\frac{1}{r^{2 \beta} \rho_{*}(r)} \int_{r}^{\infty} \mathrm{d} r^{\prime} r^{2 \beta} \rho_{*}\left(r^{\prime}\right) \mathrm{d} \phi / \mathrm{d} r$,

subject to the boundary condition that $\lim _{r \rightarrow \infty} r^{2 \beta} \rho_{*} \sigma_{r, *}^{2}=0$ [e.g. 10, 197].

\subsection{Measurements with assumed or externally cali- brated anisotropy}

Based on distances and radial velocities of 240 tracer objects in the stellar halo of our MW, including BHB stars, 
red giant stars, globular clusters and satellite galaxies, from the spectroscopic Spaghetri survey, Battaglia et al. [10] used Eqn. 13 to constrain the mass of our MW in 2005. Constant $\beta$ was adopted in their analysis. The density profile of tracers was measured to have a power-law form of $\rho_{*}(r) \propto r^{-\alpha}$, with $\alpha \sim 3.5$ out to $\sim 50 \mathrm{kpc}$ from the Galactic center [269, 412]. Assuming the power-law density profile of tracers is valid out to large distances, the radial velocity dispersion was measured to be almost a constant of $120 \mathrm{~km} / \mathrm{s}$ out to $30 \mathrm{kpc}$ and continuously drops to $\sim 50 \mathrm{~km} / \mathrm{s}$ at $120 \mathrm{kpc}$. The best-fit NFW model led to the virial mass of our MW of $M_{200}=0.7_{-0.2}^{+1.2} \times 10^{12} \mathrm{M}_{\odot}$, and the best-fit mass within $120 \mathrm{kpc}$ to the Galactic center was constrained to be $5.4_{-1.4}^{+2.0} \times 10^{11} \mathrm{M}_{\odot}$.

In addition to a constant $\beta$, Battaglia et al. [10] investigated alternative functional forms of $\beta$ profiles as a function of the Galactocentric distance. For a given mass model, although not all functional forms of $\beta$ can produce reasonable fits to the data, the best-fit virial mass is strongly dependent of the chosen functional form for $\beta$ (see also, e.g., [23] and [424]). In a follow-up study, Dehnen et al. in 2006 [100] revisited the results of Battaglia et al. [10], and found a virial mass of about $1.5 \times 10^{12} \mathrm{M}_{\odot}$. In contrast to Battaglia et al. [10], Dehnen et al. [100] claimed that the observed radial velocity dispersions are consistent with a constant velocity anisotropy of tracers, if the density profile of tracers is truncated beyond $160 \mathrm{kpc}$. These studies demonstrate that the mass to be constrained is very sensitive to assumptions behind both velocity anisotropies and tracer density profiles.

Given the strong $\beta$-dependence, some other studies attempted to rely on numerical simulations to estimate the anisotropy when applying the Jeans equation. Xue et al. in 2008 [411] also adopted the SJE as part of their analysis, but instead of directly fitting the observed radial velocity dispersions with assumptions of $\beta$, Xue et al. [411] constrained the rotation curve of our MW out to $60 \mathrm{kpc}$, which relies on the distribution of radial versus circular velocities of star particles in two simulated halos of hydrodynamical simulations. The circular velocity as a function of radius within $60 \mathrm{kpc}$ was determined by matching the observed distribution of radial versus circular velocities to the corresponding distribution in simulated halos. In their analysis, the SJE was used to scale their simulated halos, which have slightly different radial profiles of star particles compared with the best estimated power-law slope of our MW. The mass within $60 \mathrm{kpc}$ to the Galactic center was estimated to be $4.0 \pm 0.7 \times 10^{11} \mathrm{M}_{\odot}$. Adopting the NFW model profile, the virial mass was constrained to be $M_{200}=0.84_{-0.2}^{+0.3} \mathrm{M}_{\odot}$.

Based on halo stars with radial velocity measurements from the Hypervelocity Star Survey, Gnedin et al. in 2010 [153] measured the radial velocity dispersion between 25 and
$80 \mathrm{kpc}$ from the Galactic center. The velocity anisotropy was inferred from numerical simulations, with a plausible range of $0 \leqslant \beta \leqslant 0.5$ and a most likely value of 0.4 . Over the probed radial range, the power-law index of the tracer density profile was between 3.5 and 4.5 . The plausible range of circular velocity at $80 \mathrm{kpc}$ inferred from the SJE was between 175 and $231 \mathrm{~km} / \mathrm{s}$. Gnedin et al. [153] constrained the mass within $80 \mathrm{kpc}$ to the Galactic center as $6.9_{-1.2}^{+3.0} \times 10^{11} \mathrm{M}_{\odot}$. The virial mass within $300 \mathrm{kpc}$ was extrapolated to be $M_{200}=1.3 \pm 0.3 \times 10^{12} \mathrm{M}_{\odot}$.

Very recently, Zhai et al. in 2018 [424] used the SJE to model the differentiation of line-of-sight velocity dispersions based on $9627 \mathrm{~K}$ giant stars from LAMOST DR5, with distances between 5 and $120 \mathrm{kpc}$ from the Galactic center. If $\beta$ was assumed as 0.3 , the MW virial mass was constrained to be $1.11_{-0.20}^{+0.24} \times 10^{12} \mathrm{M}_{\odot}$.

\subsection{Inferring mass and anisotropy from data}

To overcome the mass-anisotropy degeneracy, many studies devoted efforts to either directly infer or indirectly model $\beta$ from observational data. In the solar vicinity, $\beta$ was measured to be $\sim 0.6$ based on proper motions of stars [e.g. 37, 356]. Without proper motions, tangential velocities with respect to the Galactic center can still be inferred from the line-ofsight velocities for objects in the inner MW, given the fact that our Sun is about $8 \mathrm{kpc}$ from the Galactic center [e.g. 91, 93, 197, 208, 354]. The observed line-of-sight velocities are contributed by both radial and tangential velocities with respect to the Galactic center. The fraction of tangential velocities contained in the line-of-sight velocities depends on both Galactocentric distances and Galactic coordinates of the observed object [208]. For tracer objects at large distances, the line-of-sight velocities are dominated by the radial components and contain very little information about the tangential velocity components.

Early in 1997, Sommer-Larsen et al. [364] analyzed 679 BHB stars between 7 and $65 \mathrm{kpc}$ from the Galactic center. Assuming dynamical equilibrium in a logarithmic Galactic potential, they found indications that the tangential velocity dispersion further beyond the solar radius should be larger than the value in our solar neighborhood. In 2005, Sirko et al. [354] fitted an ellipsoidal velocity distribution to $1170 \mathrm{BHB}$ stars from SDSS, and reported that the halo beyond our solar vicinity is close to isotropic. Adopting a power-law distribution function with a constant $\beta$ (see Sec. 5 for more details of the distribution function), Deason et al. in 2011 [91] fitted 3549 BHB stars from SDSS/DR7 and reported a tangential halo between 10 and $25 \mathrm{kpc}$ and a radial halo between 25 and $50 \mathrm{kpc}$. Then in a later study, Deason et al. in 2012 [93] allowed both the potential parameter and velocity anisotropy in 
their model distribution function to vary and reported $\beta=0.5$ between 16 and $48 \mathrm{kpc}$. It was discussed by Kafle et al. in 2012 [197] that the tangential halo claimed by Deason et al. in 2011 [91] within $25 \mathrm{kpc}$ is very likely due to the broad radial binning.

In fact, the study by Kafle et al. in 2012 [197] involved modeling of the anisotropy profile between 9 and $25 \mathrm{kpc}$ based on maximum likelihood analysis with analytical distribution functions. The best constrained $\beta$ is close to 0.5 in the very inner part of our MW and falls sharply beyond $13 \mathrm{kpc}$, reaching a minimum of -1.2 at $17 \mathrm{kpc}$ and rises again on larger scales. Beyond $25 \mathrm{kpc}$, radial velocities can still be measured, but it was impossible to properly measure the tangential components from their line-of-sight velocities. Kafle et al. [197] fitted three-component potential models of Galactic disk, bulge and halo to the estimated circular velocity profile out to $25 \mathrm{kpc}$ based on the SJE, and the mass enclosed within $25 \mathrm{kpc}$ was measured to be $2.1 \times 10^{11} \mathrm{M}_{\odot}$. The virial mass was extrapolated to be $M_{200}=0.77_{-0.30}^{+0.40} \times 10^{12} \mathrm{M}_{\odot}$. With the extrapolated potential profile, tracer density profile and the measured radial velocity dispersion on distances larger than what can be probed by their sample of tracers, they used the SJE to predict $\beta$ to be roughly 0.5 over the radial range of 25 to $56 \mathrm{kpc}$.

Note, however, the very inner region of our MW, which is close to the Galactic disk, is not spherically symmetric, but the SJE assumes spherical symmetry. This can bias the estimated mass. In addition, the underlying potential of the outer halo is not ideally spherical because dark matter halos are triaxial [194]. There are efforts of applying the SJE to simulated galaxies and halos [e.g. 199, 391]. More importantly, the assumption of a steady-state is also non-trivial and can lead to significant systematics. For example, Wang et al. in 2018 [391] have shown evidences of how violations of the spherical and the steady state assumptions behind the SJE can potentially affect the constrained halo mass of MW-like halos. We provide more detailed discussions in Sec. 10.2.

Using proper motions of 13 main sequence halo stars from the multi-epoch HST/ACS photometry, Deason et al. in 2013 [97] reported that $\beta$ is consistent with zero (isotropic) at $24 \mathrm{kpc}$. In addition, King III et al. in 2015 [208] found a minimum in their measured anisotropy profiles at $\sim 20 \mathrm{kpc}$, based on 6174 faint F-type stars from the radial velocity sample of the MMT telescope, and 13480 F-type stars from SDSS. However, compared with Kafle et al. [197], the minimum in their anisotropy profile is more negative, and they claimed that the less negative measurements in other studies is likely due to their broader binning.

Direct measurements of $\beta$ and the mass distribution up to and beyond the Galactocentric distance of $100 \mathrm{kpc}$ are even more challenging, where the line-of-sight velocities are almost entirely dominated by the radial velocities with respect to the Galactic center. Using a sample of halo stars out to $\sim 150 \mathrm{kpc}$, Deason et al. in 2012 [95] found that the radial velocity dispersion of these stellar tracers falls rapidly on such large distances. Assuming the tracer density falls off between 50 and $150 \mathrm{kpc}$ with a power-law index smaller than 5 and assuming radial orbits, the mass within $150 \mathrm{kpc}$ to the Galactic center was estimated to lie in the range between $5 \times 10^{11}$ and $10^{12} \mathrm{M}_{\odot}$.

In a later study by Kafle et al. in 2014 [198], the radial velocity dispersion profile out to $\sim 160 \mathrm{kpc}$ was measured with $\mathrm{K}$ giants from SDSS/DR9, and the SJE was used to constrain the mass distribution and the velocity anisotropy out to such large distances. Kafle et al. [198] modeled the inner tracer density profile as double power law with a break radius. Beyond $100 \mathrm{kpc}$, the profile was assumed to be truncated beyond a characteristic radius plus an exponential softening quantified by some scale length. Within $25 \mathrm{kpc}, \beta$ can be known from previous studies. Beyond $50 \mathrm{kpc}$, they assumed $\beta$ to be a constant, and the change of $\beta$ was assumed to be linear between 25 and $50 \mathrm{kpc}$. The break and truncation radii, softening scale length and the constant $\beta$ beyond $50 \mathrm{kpc}$ were all treated as free parameters, in combination with other free parameters in their three-component potential model. The virial mass was best fit to be $M_{200}=0.71_{-0.16}^{+0.31} \times 10^{12} \mathrm{M}_{\odot}$, and $\beta$ of the outer halo was estimated to be $0.4 \pm 0.2$.

Using multiple species of halo stars and combining the terminal velocity measurements with the SJE analysis, Bhattacharjee et al. in 2014 [23] measured the rotation curve of our MW up to $\sim 200 \mathrm{kpc}$. Since the circular velocity decreases with the increase of $\beta$ at a given radius, the maximum value of $\beta=1$ corresponds to the lower limit of mass enclosed within $200 \mathrm{kpc}$, which was constrained by Bhattacharjee et al. [23] to be $6.8 \pm 4.1 \times 10^{11} \mathrm{M}_{\odot}$.

Huang et al. in 2016 [189] used about 16,000 primary red clump giants in the outer disk from the LSS-GAC (LAMOST Spectroscopic Survey of the Galactic Anticancer ) of the ongoing LAMOST experiment and the SDSS-III/APOGEE survey, plus 5,700 K giants from the SDSS/SEGUE survey to derive the rotation curve of our MW out to $100 \mathrm{kpc}$. In the inner MW region, the rotation velocity was deduced from line-ofsight velocities following the approaches in Sec. 3, whereas the rotation curve in the outer halo was obtained from the SJE, with the values of $\beta$ taken from all the previous studies mentioned above and interpolated. Their best-fit potential model led to the virial mass of $M_{200}=0.85_{-0.08}^{+0.07} \times 10^{12} \mathrm{M}_{\odot}$.

In 2017, Ablimit and Zhao [2] adopted 860 ab-type RR Lyrae stars in the Galactic halo to look at the rotation velocities out to $\sim 50 \mathrm{kpc}$ using the SJE. Their sample of stars 
were identified from the Catalina Surveys DR1, combined with spectroscopic data from SDSS DR8 and LAMOST DR4 to obtain radial velocities. They adopted two different choices of $\beta$, a constant value of $\beta=0$ within $50 \mathrm{kpc}$ and the radiusdependent $\beta$ profile by [402] (see Sec. 5.2 for more details), in which $\beta$ changes from $\sim 0.32$ to $\sim 0.67$ between 10 and $50 \mathrm{kpc}$. For $\beta=0$, the circular velocity at $50 \mathrm{kpc}$ was constrained to be $180.00 \pm 31.92 \mathrm{~km} / \mathrm{s}$, and the enclosed mass within $50 \mathrm{kpc}$ was estimated as $3.75 \pm 1.33 \times 10^{11} \mathrm{M}_{\odot}$.

More recently, since much more proper motion data has been measured by Gaia, Bird et al. in 2019 [30] directly measured $\beta$ for more than 8,600 metal poor K giants, based on distances and line-of-sight velocities from LAMOST and cross matched to Gaia DR2 to obtain proper motions. Between the solar radius and $25 \mathrm{kpc}$ to the Galactic center, their sample is highly radial $(\beta \sim 0.8) . \beta$ gradually becomes less radial beyond $25 \mathrm{kpc}$, reaching $\sim 0.3$ at $\sim 100 \mathrm{kpc}$. In contrast to previous measurements made by Kafle et al. in 2012 [197], Deason et al. in 2013 [97] and King III et al. in 2015 [208], Bird et al. [30] did not report any minimum for the $\beta$ profile within $25 \mathrm{kpc}$. In addition, they claimed the sensitivity of their measured $\beta$ profile to substructures.

To conclude, the measurement of the velocity anisotropy or $\beta$ profile, from the Galactic center to the outer stellar halo, still suffers from inconsistencies and debates. The robustness of the measurements depends on a variety of factors. First of all, whether proper motions are available is very important, and deriving $\beta$ from line-of-sight velocities might suffer from systematics due to the assumed tracer velocity models. As we have already mentioned, many previous studies constrained the tangential velocity components from the observed lineof-sight velocities have to rely on fitting ellipsoidal models in velocity space or distribution functions in phase space. Moreover, the particular type of tracer objects and the sample selection may both result in different measurements, because different tracer populations are not expected to have the same velocity distributions. Also, the influence of substructures is still uncertain. This has been discussed by Loebman et al. in 2018 [242], in which the possible origin and persistence of the dip feature in $\beta$ profiles were investigated using both $\mathrm{N}$-body and hydrodynamical simulations.

\subsection{Variants to the SJE: Mass estimators}

The Jeans equation itself can be regarded as a mass estimator in which the enclosed mass is related to the velocity dispersion profile. Starting from the collisionless Boltzmann equation or the SJE, alternative forms of mass estimators may be derived, usually under some more specific forms of the potential and tracer profiles. These estimators may be more compact and convenient to apply to data than the SJE. However, one should bear in mind that the limitations and caveats in the Jeans modeling as discussed above are generally also relevant to these alternative mass estimators, in addition to modelspecific systematics in case extra assumptions are made to derive the estimator.

Early attempts of deriving mass estimators which relate observed positions and velocities of tracer objects to the enclosed mass can be traced back to the 1960s, which is called the virial theorem [237]. The virial theorem estimator, however, results in a biased estimate of the true mass as pointed out by Bahcall and Tremaine in 1981 [5].

In 2010, Watkins et al. [393] derived mass estimators for scale-free (single power law) potentials and tracer density profiles, which relate the enclosed mass within the maximum radius of the tracer sample to the observed velocities and distances of these tracers. When tangential velocities are unknown due to missing proper motions, the estimator relates the average radial velocities and distances of tracers to the enclosed mass, while the velocity anisotropy of tracers, the power-law indexes of the potentials and tracer density profiles have to be assumed in advance.

Applying the derived mass estimator to satellite galaxies in our MW and assuming isotropic velocity anisotropy $(\beta=0)$, the mass within $300 \mathrm{kpc}$ was estimated to be $1.17 \pm 0.3 \times 10^{12} \mathrm{M}_{\odot}$ by Watkins et al. [393]. In their analysis, six satellites have proper motions. Two estimators with or without tangential velocities when proper motions are available or not have been applied separately. The final result was a weighted average between results from the two estimators, and Monte Carlos simulations were adopted to estimate the measurement errors. The estimated mass within $300 \mathrm{kpc}$ dropped by $\sim 60 \%$ if only radial velocities were used. If considering the plausible range of anisotropies based on both numerical simulations and observations, the uncertainty was in fact very large, ranging from $\sim 1.0$ to $\sim 2.7 \times 10^{12} \mathrm{M}_{\odot}$. This corresponds to the large errorbar in Fig. 1. In their analysis Leo I played 27\% of role compared with the other satellites.

Watkins et al. [393] drew the plausible power-law index value for their potential model by looking at the best-fit slopes of NFW profiles over the radial range of their satellites. For MW-analogous concentrations and virial radii, the power-law index of the potential changes slowly and is close to 0.5 . However, the NFW profile, which can be used to well approximate the dark matter halo profiles in modern cosmological simulations, does not produce a strictly single power law potential outside $10 \mathrm{kpc}$. In a few follow-up papers, mass estimators with more generalized potentials have been further developed [e.g. 3, 4, 125].

In fact, not only the potential profile, but also the tracer density profile is not a single power law. Many late-time 
studies found breaks in the number density profiles of halo stars at $\sim 16$ to $\sim 30 \mathrm{kpc}$ [e.g. 12, 88, 92, 139, 198, 313, 345, 346, 394, 410]. The power-law indexes reported by many of these studies are shallower within the breaking radii, and become steeper outside. For example, [12] found values of $\alpha=2$ and $\alpha=4$ for inner and outer profiles. [394] investigated RR Lyrae stars out to $100 \mathrm{kpc}$ and found values of 2.4 and 4.5. [92] reached similar conclusions (power-law indexes of 2.3 and 4.6) using BHB stars out to $40 \mathrm{kpc}$. Using main-sequence turnoff stars from the Canada-France-Hawaii Telescope Legacy Survey, [346] reported a slightly shallower outer slope of 3.8 beyond $28 \mathrm{kpc}$, and in a follow-up paper, [345] found a smaller breaking radius of $16 \mathrm{kpc}$. The tension among different measurements might be due to significant variations of the power-law index values over the sky, in that substructures can potentially affect the measured values, especially if one relies on narrow pencil-beam surveys [e.g. 243]. It was pointed out by Lowing et al. in 2013 [94] that the break in tracer density profiles is likely associated with an early and massive accretion event.

More recently, Sohn et al. in 2018 [363] adopted proper motion measurements from HST for globular clusters between 10 and $40 \mathrm{kpc}$ from the Galactic center. Still based on the mass estimator developed by Watkins et al. in 2010 [393] (the one with proper motion and based on scale-free potential and tracer density profiles), Sohn et al. [363] constrained the mass within $39.5 \mathrm{kpc}$ to the Galactic center as $6.1_{-1.2}^{+1.8} \times 10^{11} \mathrm{M}_{\odot} . \beta$ was measured from their sample of globular clusters as $0.609_{-0.229}^{+0.130}$. The virial mass was extrapolated to be $M_{200}=1.71_{-0.79}^{+0.97} \times 10^{12} \mathrm{M}_{\odot}$.

Using 34 globular clusters with proper motions from the second data release (DR2) of Gaia, together within the sample of globular clusters from HST [363], Watkins et al. in 2019 [395] further estimated the mass within $21.1 \mathrm{kpc}$ and $39.5 \mathrm{kpc}$ to the Galactic center as $2.1_{-0.3}^{+0.4} \times 10^{11} \mathrm{M}_{\odot}$ and $4.2_{-0.6}^{+0.7} \times 10^{11} \mathrm{M}_{\odot}$, respectively. The virial mass was extrapolated to be $M_{200}=1.29_{-0.44}^{+0.75} \times 10^{12} \mathrm{M}_{\odot} . \beta$ was estimated from the data as $0.52_{-0.14}^{+0.11}$.

A very recent study by Fritz et al. [138] applied the Watkins et al. mass estimator to 45 satellites with Gaia proper motions. Subhalos from dark matter only simulations are used to test and calibrate their measurements. A significant bias has been reported after applying the mass estimator to simulations, which was mainly attributed to the deviation of satellite density profiles from a single power law. Systematic uncertainties arising from LMC mass and LMC satellites were also discussed. The mass enclosed within 64 and $273 \mathrm{kpc}$ was estimated to be $5.8_{-1.4}^{+1.5} \times 10^{11} \mathrm{M}_{\odot}$ and $14.3_{-3.2}^{+3.5} \times 10^{11} \mathrm{M}_{\odot}$, taking into account potential influences of the LMC. The mass out to $\sim 308 \mathrm{kpc}$ was extrapolated to be

$$
1.51_{-0.40}^{+0.45} \times 10^{12} \mathrm{M}_{\odot}
$$

\section{Distribution functions: halo stars, globular clusters and satellite galaxies}

As we have introduced in Section 4, the phase-space distribution function fully specifies the dynamical structure of the system, and in principle contains the most complete information for dynamical modeling. As solutions to the collisionless Boltzmann equation (Equation 10), these functions connect the phase-space coordinates of tracers to the underlying potential, and thus can be used to fit to the observed positions and velocities of tracer objects and constrain the model potential. In the following, we discuss how available functional forms can be chosen, in terms of classical integrals of motion or actions, and we will introduce the efforts of using the distribution functions to constrain the mass of our MW. In addition, we also review the class of simulation-based distribution functions by linking observed MW satellite galaxies to simulated subhalos and the set of orbital probability distributions. Measurements in this section fall in the category of "DF" in Fig. 1.

\subsection{Distribution function in terms of classical integrals of motion}

The phase-space distribution function, $f(\mathbf{x}, \mathbf{v}, t)$, describes a dynamical system in terms of positions, $\mathbf{x}$, velocities, $\mathbf{v}$, and time, $t$. As we have mentioned in Sec. 4, the system is independent of time when it is in steady state. The Jeans theorem states that the steady state solution of the distribution function is connected to positions and velocities only through the integrals of motions. The strong Jeans theorem further states that there are only three independent integrals of motions. For systems with a spherical symmetry, the distribution function of steady state systems can be written down in terms of energy and the magnitude of angular momentum, i.e., $f(E, L)$ [248].

The phase-space distribution function of tracer objects bound to the underlying potential (binding energy $E>0$ ) can be described by the Eddington formula [116], which is basically the Abell inversion of the tracer density profile, $\rho_{*}(r)$, under the spherical assumption. The simplest isotropic and spherically symmetric case is

$F(E)=\frac{1}{\sqrt{8} \pi^{2}} \frac{\mathrm{d}}{\mathrm{d} E} \int_{\Phi\left(r_{\text {max }, t}\right)}^{E} \frac{\mathrm{d} \rho_{*}(r)}{\mathrm{d} \Phi(r)} \frac{\mathrm{d} \Phi(r)}{\sqrt{E-\Phi(r)}}$,

where the distribution function only depends on the binding energy per unit mass, $E=\Phi(r)-\frac{v^{2}}{2}$. $\Phi(r)$ and $\frac{v^{2}}{2}$ are the underlying dark matter halo potential and kinetic energy per 
unit mass of tracers. The integral goes from the potential at the tracer boundary to the binding energy of interest ${ }^{7)}$. Usually both the zero point of potentials and the tracer boundary, $r_{\text {max,t }}$, are chosen at infinity, and thus $\Phi\left(r_{\text {max }, \mathrm{t}}\right)=0$.

In reality the velocity distribution of tracers is anisotropic. One possible anisotropic form introduces an angular momentum $(L)$ dependence as

$F(E, L)=L^{-2 \beta} f(E)$,

where the energy part, $f(E)$, is expressed as [85]

$$
\begin{array}{r}
f(E)=\frac{2^{\beta-3 / 2}}{\pi^{3 / 2} \Gamma(m-1 / 2+\beta) \Gamma(1-\beta)} \times \\
\frac{\mathrm{d}}{\mathrm{d} E} \int_{\Phi\left(r_{\text {max }, \mathrm{t}}\right)}^{E}(E-\Phi)^{\beta-3 / 2+m} \frac{\mathrm{d}^{m}\left[r^{2 \beta} \rho_{*}(r)\right]}{\mathrm{d} \Phi^{m}} \mathrm{~d} \Phi \\
=\frac{2^{\beta-3 / 2}}{\pi^{3 / 2} \Gamma(m-1 / 2+\beta) \Gamma(1-\beta)} \times \\
\int_{\Phi\left(r_{\text {max }, \mathrm{t}}\right)}^{E}(E-\Phi)^{\beta-3 / 2+m} \frac{\mathrm{d}^{m+1}\left[r^{2 \beta} \rho_{*}(r)\right]}{\mathrm{d} \Phi^{m+1}} \mathrm{~d} \Phi,
\end{array}
$$

which assumes the energy, $E$, and the magnitude of angular momentum, $L$, are separable. Here $\beta$ is the velocity anisotropy parameter. $m$ is an integer chosen to make the integral converge and depends on the value of $\beta$. If the allowed region of $\beta$ is $-0.5<\beta<1$, then $m=1$.

When the tangential velocities of tracers are not available, the phase-space distribution in terms of radius, $r$, and radial velocity, $v_{r}$, is given by the integral over tangential velocities, $v_{t}$,

$P\left(r, v_{r} \mid C\right)=\int L^{-2 \beta} f(E) 2 \pi v_{t} \mathrm{~d} v_{t}$,

where $C$ denotes a set of model parameters. Via the Laplace transform, this can be written as

$P\left(r, v_{r} \mid C\right)=\frac{1}{\sqrt{2} \pi r^{2 \beta}} \int_{\Phi\left(r_{\text {max }, \mathrm{t}}\right)}^{E_{r}} \frac{\mathrm{d} \Phi}{\left(E_{r}-\Phi\right)^{1 / 2}} \frac{\mathrm{d} r^{2 \beta} \rho_{*}}{\mathrm{~d} \Phi}$,

where $E_{r}=\Phi(r)-v_{r}^{2} / 2$. All factors of $m$ cancel in the Laplace transform and hence Eqn. 18 does not depend on $m$.

The more explicit form of the above phase-space distribution function depends on the chosen model for the underlying potential $(\Phi)$ and tracer density $\left(\rho_{*}\right)$ profiles. Free parameters in the potential and tracer models are often constrained through the Bayesian framework.
Early attempts of using such distribution functions to constrain the mass of our MW can be traced back to a few decades ago. In 1987, Little and Tremaine [239] devised the method of fitting the model distribution function to observed positions and velocities of satellite galaxies under the Bayesian framework, to constrain the mass within $50 \mathrm{kpc}$ to the Galactic center. In 1989, Zaritsky et al. [422] repeated the analysis with improved velocity estimates of satellites. Assuming a point mass, the median mass of the Galaxy was estimated to be $9.3_{-1.2}^{+4.1} \times 10^{11} \mathrm{M}_{\odot}$ for radial satellite orbits, and $12.5_{-3.2}^{+8.4} \times 10^{11} \mathrm{M}_{\odot}$ for isotropic satellite orbits. The estimated mass can be significantly smaller if excluding Leo I from their sample of satellite galaxies.

Slightly later in 1991, similar estimates were reached by Norris and Hawkins [284] and by Kulessa and Lynden-Bell in 1992 [220]. Then in 1996, Kochanek [213] applied such phase-space distribution functions to constrain the mass of our MW using the Jaffe model, in combination with other approaches including the local escape velocity of stars (see Sec. 2), which suppressed the low-velocity solutions, and the rotation curve of the disk (see Sec. 3), which eliminated solutions predicting too high rotation velocities at the solar neighborhood. The Local Group timing argument (see Sec. 7 for details) was also adopted to suppress high-mass solutions. Kochanek [213] discussed cases when only radial velocities were available and when both radial and tangential velocities were available. The median mass within $50 \mathrm{kpc}$ was estimated to be $5.1_{-1.1}^{+1.3} \times 10^{11} \mathrm{M}_{\odot}(90 \%$ confidence level and with Leo I).

Wilkinson and Evans in 1999 [400] adopted the truncated flat-rotation model potential in their distribution function, and used the distances and velocities of satellite galaxies and globular clusters to constrain the model ( 27 objects in total and 6 with proper motions). Assuming the density profile of satellites and globular clusters was a power law with index of 3.4 and by including Leo I, the mass enclosed within $50 \mathrm{kpc}$ was estimated to be $5.4_{-3.6}^{+0.2} \times 10^{11} \mathrm{M}_{\odot}$, and the total mass of the halo was estimated as $1.9_{-1.7}^{+3.6} \times 10^{12} \mathrm{M}_{\odot}{ }^{8)}$.

The estimates were updated by Sakamoto et al. in 2003 [324] using a larger sample of dynamical tracers including 11 satellites, 127 globular clusters and 413 field horizontal branch stars, among which half of the objects had proper motions. The total mass was constrained to be $2.5_{-1.0}^{+0.5} \times 10^{12} \mathrm{M}_{\odot}$ if including Leo I and $1.8_{-0.7}^{+0.4} \times 10^{12} \mathrm{M}_{\odot}$ if excluding Leo I. The mass within $50 \mathrm{kpc}$ was estimated to be $5.5_{-0.2}^{+0.0} \times 10^{11} \mathrm{M}_{\odot}$ if including Leo I and $5.4_{-0.4}^{+0.1} \times 10^{11} \mathrm{M}_{\odot}$ without Leo I.

Later in 2012, Deason et al. [93] adopted the single power-

\footnotetext{
7) To define the binding energy, we adopt the convention that $\Phi\left(r_{\max , t}\right)=0$, and $\Phi(r)>0$, which differs from the potential used in previous sections by a sign.

8) The total mass is a free parameter in their truncated flat-rotation potential model. The errors are determined by both the small sample size and the measurement errors, which are thus very large.
} 
law potential and tracer density profiles in the model distribution function proposed by [127], and considered a constant flattening of the halo, by using 1933 blue horizontal branch stars within 18 and $48 \mathrm{kpc}$ from the Galactic center as dynamical tracers. The mass within $50 \mathrm{kpc}$ was constrained to be $4.2 \pm 0.4 \times 10^{11} \mathrm{M}_{\odot}$. Combined with the measured rotation velocities and enclosed masses within fixed radii smaller than the virial radius from other studies, [93] claimed that their results favored an NFW halo with concentration of about 20 . We extrapolated the NFW profile to get the virial mass of $M_{200}=0.94_{-0.20}^{+0.22} \times 10^{12} \mathrm{M}_{\odot}$.

In a series of papers since 2015 [111, 112, 113, 114, 115], the distribution functions were revisited. In their first paper, Eadie et al. in 2015 [114] used the Hernquist and the isotropic Jaffe models in their analysis to model the incomplete data. Their sample of dynamical tracers involved 59 globular clusters and 29 dwarf galaxies, out of which 26 globular clusters and 18 dwarf galaxies did not have proper motions. For tracers with large enough Galactocentric distances, their observed line-of-sight velocities can be approximated as radial velocities with respect to the Galactic center, while the unknown tangential velocities were treated as nuisance parameters, with the parameter space sampled through the hybrid-Gibbs sampler. The total mass of our MW, which is a parameter in the Hernquist model, was estimated as $1.55_{-0.13}^{+0.18} \times 10^{12} \mathrm{M}_{\odot}(95 \%$ confidence region, isotropic anisotropy). The mass within $260 \mathrm{kpc}$ was constrained as $1.37_{-0.10}^{+0.14} \times 10^{12} \mathrm{M}_{\odot}(95 \%$ confidence region $)$. It was found that since the proper motion of Pal 3 has very large uncertainties, Pal 3 played a very important weight in determining the mass of our MW. If excluding Pal 3 in their analysis, the MW mass through the Hernquist parameter was best constrained as $1.36_{-0.10}^{+0.15} \times 10^{12} \mathrm{M}_{\odot}(95 \%$ confidence region $)$.

The first paper [114] was based on simple potential models and it was assumed that the dynamical tracers and the underlying dark matter have the same spatial distributions. In a follow-up study of 2016, Eadie et al. [113] adopted powerlaw potential and tracer density profiles of [127] and [93], and adopted different radial distributions for tracers and dark matter. They used globular clusters only as tracers, because a single power-law density profile better models a single population of objects. The catalog of $[174,175]$ were used as the starting point of their sample globular clusters, which contains 157 objects. After exclusions, in total 89 globular clusters were selected, out of which 18 did not have complete velocity measurements. A series of different scenarios were tried, by choosing to either fix or free the parameters for the underlying potential profile, the tracer density profile and the velocity anisotropy. When all the parameters were free, the mass within $125 \mathrm{kpc}$ was constrained to be $5.22_{-0.43}^{+0.41} \times 10^{11} \mathrm{M}_{\odot}$
(50\% confidence). Extrapolated to the virial radius, the virial mass was found to be $M_{200}=0.682_{-0.076}^{+0.071} \times 10^{12} \mathrm{M}_{\odot}(50 \%$ confidence region). If only using globular clusters outside $10 \mathrm{kpc}$ where a single-power law potential model can better approximate the outer slope represented by the NFW model, the virial mass was constrained as $M_{200}=0.902_{-0.333}^{+0.184} \times 10^{12} \mathrm{M}_{\odot}$ (50\% confidence $)$.

Eadie et al. in 2017 [115] further applied the hierarchical Bayesian approach to model the phase-space distribution of globular clusters, which includes more meaningful treatment of measurement errors. With the sample of globular clusters from their previous study in 2016 [113], the mass within $125 \mathrm{kpc}$ to the Galactic center was measured as $6.3 \pm 1.1 \times 10^{11} \mathrm{M}_{\odot}(95 \%$ confidence $)$. The virial mass was extrapolated to be $M_{200}=0.86_{-0.19}^{+0.23} \times 10^{12} \mathrm{M}_{\odot}(95 \%$ confidence). Further extrapolated to $300 \mathrm{kpc}$, the enclosed mass within $300 \mathrm{kpc}$ was found to be $1.14 \pm 0.22 \times 10^{12} \mathrm{M}_{\odot}$.

More recently, the Gaia team measured and released the mean proper motions through member stars for 75 globular clusters [142], which cover about half of the objects previously provided by [174] and [175]. In their 2019 paper, Eadie et al. [111] replaced the data of [174] and [175] by the Gaia proper motions with some exceptions. When proper motions were not available from Gaia, the HST proper motions [363], if available, were adopted. If the proper motions were still missed, the measurements from other studies (see [113] for details) were adopted. In total, the sample contained $154 \mathrm{ob}$ jects, out of which 52 had incomplete measurements. In the mean time, both Vasiliev [383] and Baumgardt et al. [11] in 2019 identified member stars for the $\sim 150$ globular clusters from [174] and [175], and provided independent mean proper motion estimates for all of them. Eadie \& Jurić in 2019 [111] afterwards made their measurements using both their own extended globular cluster sample based on Gaia DR2 plus HST and the catalog of Vasiliev [383]. The two catalogs gave very similar constraints. Based on globular clusters with Galactocentric distances larger than $15 \mathrm{kpc}$, the median estimate of the $\mathrm{MW}$ virial mass was $M_{200}=0.70_{-0.12}^{+0.17} \times 10^{12} \mathrm{M}_{\odot}(68 \%$ confidence reading from their Fig. 7). If further excluding 4 globular clusters with Galactocentric distances between 15 and $20 \mathrm{kpc}$ from [271], the measurement became slightly larger $M_{200}=0.77_{-0.16}^{+0.25} \times 10^{12} \mathrm{M}_{\odot}$. They provided the cumulative mass profile out to slightly beyond $\sim 100 \mathrm{kpc}$.

As the readers may have found, most of the measurements adopted simplified potential models such as the truncated flat rotation and power-law potentials. However, the NFW profile, which well approximates the dark matter halo profiles in cosmological simulations, lead to more complicated analytical forms of the phase-space distribution function [392], and have been evaluated numerically [e.g. 399], but have never 
been applied to real tracer objects.

The performance of single power-law potential models and the functional form of Eqn. 15 both await further tests. Moreover, the incompleteness of available data and measurement errors can both affect the results. There have been studies which applied such phase-space distribution functions to simulated galaxies and dark matter halos to test their performances [e.g. 112, 392, 406], for which we make more detailed discussions in Sec. 10.2. Briefly, Eadie et al. in 2018 [112] showed that the model has difficulties predicting both the inner and outer regions of the true underlying cumulative mass profiles of simulated galaxies, after using tracers at all Galactocentric distances. By extending the distribution function to be based on the NFW profile, Wang et al. in 2015 [392] demonstrated different levels of systematic biases from halo to halo. Deviations from the NFW potential, violations of the steady state assumption and invalid functional forms of the distribution function can all be responsible for such biases. On the other hand, a careful examination of the goodness of fit may serve as a way to discriminate between different models. As shown by the second paper of Li et al. in 2019 [235], it is possible to verify or select proper distribution function models for a given observation.

\section{2 distribution function in terms of actions}

Actions are defined as integrals of the generalized momenta along a path of the generalized coordinate $J_{i}=\int p_{i} \mathrm{~d} q_{i}$. The components, $J_{i}$, are linked to separable potentials. Each momentum, $p_{i}$, is a function of one coordinate, $q_{i}$, plus three integrals of motion, which are introduced through the procedure of separation upon solving the Hamilton-Jacobian equation. In a spherical potential, for example, the radial action is defined as

$J_{r}=\frac{1}{\pi} \int_{r_{p}}^{r_{a}} \mathrm{~d} r \sqrt{2 E-2 \Phi-\frac{L^{2}}{r^{2}}}$,

where $r_{p}$ and $r_{a}$ are the radii at pericenter and apocenter. The other two actions in a spherical potential can be chosen as $J_{\phi}=L_{z}$ and $L-L_{z}$, i.e., the component of the angular momentum along the $z$-axis and the difference between the magnitude of angular momentum and its $z$-component.

The spherical isochrone potential [e.g. 126, 182] is a special case that have analytical solutions to Eqn. 19. The triaxial Stäckel potentials [90] are the most general separable potentials, allowing exact evaluations of actions using a single quadrature. For all the other potentials, actions have to be evaluated numerically.

Due to the difficulties in computing actions, these quantities were not very commonly used in stellar dynamics to constrain the MW potential model in the past. In spite of the difficulties, the usage of actions is still very appealing, mainly because of two reasons. On one hand, they are adiabatically invariant, meaning that they are conserved quantities in a slowly varying potential. On the other hand, when combined with canonically conjugate angles, they can form a complete system of canonical coordinates.

The actions and their canonically conjugate angles have very useful properties. For example, finite triple of $\left(J_{r}, J_{\phi}, J_{z}\right)$ with $J_{r}>0$ and $J_{z}>0$ defines a bound orbit. Along any orbit, the canonically conjugate angle, $\theta_{i}$, increases linearly with time at a fixed rate of $\Omega_{i}(\mathbf{J})$, i.e., $\theta_{i}(t)=\theta_{i}(0)+\Omega_{i}(\mathbf{J}) t$. The phase-space volume can be expressed as $(2 \pi)^{3} \mathrm{~d}^{3} \mathbf{J}$, and the phase-space coordinates, $\mathbf{x}$, are periodic functions of $\boldsymbol{\theta}$ in the manner of $\mathbf{x}(\boldsymbol{\theta}+2 \pi k, \mathbf{J})=\mathbf{x}(\boldsymbol{\theta}, \mathbf{J})$.

More recently, a series of methods have been proposed for fast evaluations of actions in different potentials. The methods include the cylindrical adiabatic approximations [24] and refinements [26, 341], the stäckel fudge [25, 330], and the local stäckel fitting [326]. More details about these and other methods are available in a review paper of [333]. Softwares which can be used to calculate and test actions, to compute phase-space positions and velocities given actions and to infer the underlying potential given observed positions and velocities of tracers have been released as well [e.g. 27, 42, 332, 376, 382].

While evaluating actions is becoming more feasible, accurate and efficient, certain functional forms have to be proposed for the distribution of actions, in order to enable further applications to studies of Galactic disks and stellar halos. In 2010, Binney [24] discussed analytical distribution functions for the Galactic disk, and the slightly refined form discussed in a follow-up paper by Binney ad McMillan [26] is

$f\left(J_{r}, L_{z}, J_{z}\right)=f_{\sigma_{r}}\left(J_{r}, L_{z}\right) \times \frac{v_{z}}{2 \pi \sigma_{z}^{2}} e^{-v_{z} J_{z} / \sigma_{z}^{2}}$.

$f_{\sigma_{r}}\left(J_{r}, L_{z}\right)$ models the motion parallel to the disk

$f_{\sigma_{r}}\left(J_{r}, L_{z}\right)=\left.\frac{\Omega \Sigma}{\pi \sigma_{r}^{2} \kappa}\right|_{R_{c}}\left[1+\tanh \left(L_{z} / L_{o}\right)\right] e^{-\kappa J_{r} / \sigma_{r}^{2}}$.

$\Omega\left(L_{z}\right)$, which depends on $L_{z}$, is the circular frequency for the angular momentum $L_{z} . \kappa\left(L_{z}\right)$, which also depends on $L_{z}$, is the radial epicycle frequency, and $v\left(L_{z}\right)$ is its vertical counterpart. $\Sigma\left(L_{z}\right)$, which can be parametrized as $\Sigma_{0} e^{-\left(R-R_{c}\right) / R_{d}}$, is the radial surface density profile of the disk, with $R_{c}\left(L_{z}\right)$ the radius of the circular orbit with angular momentum $L_{z} . R_{d}$ is the scale length of the disk at the solar radius. The factor $\tanh \left(L_{z} / L_{0}\right)$ is a chosen odd function for rotation, where $L_{0}$ is a constant that determines the steepness of the rotation curve in the central region of solid-body rotation. On large radius 
where $r \times v_{c}$ is much larger than $L_{0}, 1+\tanh \left(L_{z} / L_{0}\right)$ simply eliminates the contribution from counter-rotating stars. $\sigma_{z}\left(L_{z}\right)$ and $\sigma_{r}\left(L_{z}\right)$ are the vertical and radial velocity dispersions, which can be parametrized as

$\sigma_{r}\left(L_{z}\right)=\sigma_{r 0} e^{q\left(R_{0}-R_{c}\right) / R_{d}}$

and

$\sigma_{z}\left(L_{z}\right)=\sigma_{z 0} e^{q\left(R_{0}-R_{c}\right) / R_{d}}$.

This was obtained by assuming that the velocity dispersions decline exponentially in radius with a scale length roughly twice that of the surface density, so $q \sim 0.5$. Binney [24] also discussed the integrated distribution function for stars with different ages in the thin disk, while for the thick disk, a single population can be assumed. The distribution function of the Galactic disk had been extended to have an analytical dependence on the metallicity of stars [331]. Moreover, perturbed distribution functions by spiral arms were discussed by other studies as well [266, 375].

The above distribution function was applied to $16,269 \mathrm{G}-$ type dwarf stars from SEGUE by Bovy and Rix in 2013 [49] to successfully infer quantities such as the mass of the disk, the total local surface density and the shape of the radial profile of dark matter halo within $12 \mathrm{kpc}$ from the Galactic center. Using 200,000 giant stars from the RAVE survey, Piffl et al. in 2014 [311] constrained the vertical density profile within $\sim 1.5 \mathrm{kpc}$ to the Galactic plane. The analytical distribution function was also used to fit the kinematics of RAVE stars and predict the vertical profile, which showed very good agreement with the observed profiles. Their results suggest that the chosen functional form of the distribution function is capable of approximating the truth.

To constrain the mass distribution of our MW out to large distances, analytical distribution functions in terms of actions for the stellar halo with double power-law tracer density profile are required. Both Posti et al. in 2015 [315] and Williams and Evans in 2015 [403] had discussed the action distribution function for double power-law tracer density profiles. The discussions were based on choosing a certain functional form for the action distribution, which can be reduced to the expected behavior of the distribution at the small and large scale regimes. In the following, we briefly introduce how the function is chosen based on the deductions made by [315].

A family of models for double power law density profiles is

$\rho_{*}(r)=\frac{\rho_{0}}{\left(r / r_{b}\right)^{\alpha}\left(1+r / r_{b}\right)^{\beta-\alpha}}$, where $\alpha$ and $\beta$ are the two power-law indexes, and $r_{b}$ is the breaking radius [28].

When $r \ll r_{b}$, the enclosed mass is $M(r) \propto r^{3-\alpha}$. Hence $\frac{\mathrm{d} \Phi}{\mathrm{d} r} \propto r^{1-\alpha}$ or $\Delta \Phi(r) \propto r^{2-\alpha}$. On the other hand, it can be proved that for a power-law potential $\Phi(r) \propto r^{a}(a=2-\alpha)$, once the position is scaled by a factor of $x^{\prime}=\xi x$, the energy and action are scaled in ways of $E^{\prime}=\xi^{a} E$ and $\mathbf{J}^{\prime}=\xi^{1+a / 2} \mathbf{J}$ respectively. Hence one can figure out that the Hamiltonian should be of the form $H(\mathbf{J})=[h(\mathbf{J})]^{a /(1+a / 2)}$, where $h(\mathbf{J})$ is some homogeneous function of $h(\xi \mathbf{J})=\xi h(\mathbf{J})$. Referring to the Poisson equation, one can have $\rho_{*} \propto|\Phi|^{1-2 / a}$ for a powerlaw potential. From the Eddington formula (Eqn. 14), it is not difficult to derive that $f(E) \propto E^{-(4+a) / 2 a}$. Considering $H(\mathbf{J})=[h(\mathbf{J})]^{a /(1+a / 2)}$ and set back $a=2-\alpha$, one can have the behavior of the double power-law distribution function on very small scales as $f(\mathbf{J})=[h(\mathbf{J})]^{-(6-\alpha) /(4-\alpha)}$.

When $r$ approaches to infinity, the potential is Keplerian, i.e., $\Phi(r) \propto r^{-1}$ and $\rho_{*} \propto|\Phi|^{\beta}$. The Hamiltonian takes the form of $H(\mathbf{J})=[g(\mathbf{J})]^{-2}$ (see [28] for more details), where $g(\mathbf{J})$ is some homogeneous function. Referring to the Eddington's formula (Eqn. 14) with $\rho_{*} \propto|\Phi|^{\beta}$, one can have $f(E) \propto$ $E^{\beta-3 / 2}$. Substituting $H(\mathbf{J})=[g(\mathbf{J})]^{-2}$ into $f(E) \propto E^{\beta-3 / 2}$, the behavior of the double-power law distribution function at infinity is $f(\mathbf{J})=[g(\mathbf{J})]^{-2 \beta+3}$.

[315] proposed the functional form to connect the two limiting behaviors above as

$f(\mathbf{J})=\frac{M_{0}}{J_{0}^{3}} \frac{\left[1+J_{0} / h(\mathbf{J})\right]^{(6-\alpha) /(4-\alpha)}}{\left[1+g[\mathbf{J}] / J_{0}\right]^{2 \beta-3}}$.

Details about the choices of homogeneous functions of $h(\mathbf{J})$ and $g(\mathbf{J})$ can be found in [404], [315] and [403]. Das and Binney in 2016 [87] have extended such distribution function to include metallicity dependence. Moreover, Binney and Wong in 2017 [29] have adopted the above disk+halo distribution functions in action space to model globular clusters in our MW.

Using the action distribution function based on double power-law density profiles developed by [403] and adopting a much simpler power-law potential, Williams and Evans in 2015 [402] constrained the enclosed mass within $50 \mathrm{kpc}$ of our MW to be $\sim 4.5 \times 10^{11} \mathrm{M}_{\odot}$, based on about 4,000 BHB stars from SDSS. Velocity anisotropy was constrained to be $\beta \sim 0.4$ at $\sim 15 \mathrm{kpc}$ and $\beta \sim 0.7$ at $\sim 60 \mathrm{kpc}$.

Following the approach of Binney and Wong in 2017 [29] and using the distribution functions for Galactic disk and the outer stellar halo described above, Posti et al. in 2019 [316] constrained the mass of our MW through the recently estimated proper motions of 75 globular clusters from Gaia DR2 and 16 other globular clusters from HST [363]. 52 globular 
clusters without proper motions from [174] were also used in their analysis. In addition to the adopted mass-concentration relation to fix the concentration parameter, Posti et al. [316] also fixed the parameters for the Galactic disk and bulge to observational constraints made by Piffl et al. in 2014 [311]. Part of the parameters in their modeling of radial and vertical velocity dispersions were fixed as well. The double power-law distribution function of the halo was simplified by fixing it to have a constant density core in phase space, and the halo was allowed to be prolated. Their results are consistent with a constant and slight radially biased halo of $\beta \sim 0.20 \pm 0.07$. The masses for the Galaxy and dark matter within $20 \mathrm{kpc}$ were constrained to be $1.91_{-0.15}^{+0.17} \times 10^{11} \mathrm{M}_{\odot}$ and $1.37_{-0.11}^{+0.12} \times 10^{11} \mathrm{M}_{\odot}$, respectively. The virial mass was extrapolated to be $M_{200}=1.1 \pm 0.3 \times 10^{12} \mathrm{M}_{\odot}$.

Based on the kinematics of member stars from Gaia DR2, Vasiliev in 2019 [383] derived proper motions for 150 globular clusters in our MW. Similar to the previous studies of Binney and Wong in 2017 [29] and Posti et al. in 2019 [316], Vasiliev [383] adopted the action distribution function to constrain the mass distribution, and found a spherical halo is preferred, with the mass enclosed inside $50 \mathrm{kpc}$ and $100 \mathrm{kpc}$ being $5.4_{-0.8}^{+1.1} \times 10^{11} \mathrm{M}_{\odot}$ and $8.5_{-2.0}^{+3.3} \times 10^{11} \mathrm{M}_{\odot}$, respectively. The virial mass was extrapolated as $M_{200}=1.0_{-0.5}^{+1.5} \times 10^{12} \mathrm{M}_{\odot}$. In the end, we note that both [316] and [383] have modeled measurement uncertainties, and have approximated the selection function of globular clusters to be complete.

Very recently, Callingham et al. in 2020 [71] investigated how the contraction of dark matter halos caused by the accumulation of baryons in the central regions can affect the action distributions. They have developed an iterative algorithm to contract dark matter halos, and have used this algorithm to predict the density and velocity distribution of the MW's contracted dark matter halo.

\subsection{Template-based distribution functions}

So far we have introduced the method of fitting a given model distribution function to the observed positions and velocities of dynamical tracers such as halo stars, satellite galaxies and globular clusters. However, this approach strongly depends on whether the adopted functional form of the model distribution is realistic or not [392]. In this subsection, we further introduce distribution functions that are generalized from simulation templates. Because the templates as well as their universality are extracted empirically from simulations, these methods fall in between dynamics based distribution function method and satellite phenomenology that we will introduce in section 8 .

The phase-space distribution of tracers can be easily extracted from simulations. However, to make these dis- tribution functions useful for dynamical inference, proper parametrization of these distribution functions are needed. It is well-known that the halo density profile can be well described by the universal NFW function parametrized by a scale density, $\rho_{s}$, and a scale radius, $r_{s}$, or equivalently a mass and a concentration parameter. Given this universality, it is natural to expect that the full phase-space distribution may also be universal, once the phase-space coordinates are scaled by appropriate combinations of NFW parameters. That is [236]

$f(\boldsymbol{r}, \boldsymbol{v})=\frac{N_{\mathrm{tot}}}{r_{s}^{3} v_{s}^{3}} \tilde{f}(\tilde{\boldsymbol{r}}, \tilde{\boldsymbol{v}})$

where the probability density in $(\tilde{\boldsymbol{r}}, \tilde{\boldsymbol{v}})$ space, $\tilde{f}(\tilde{\boldsymbol{r}}, \tilde{\boldsymbol{v}}) \equiv$ $d^{6} P / d^{3} \tilde{x} d^{3} \tilde{v}$, is approximately the same for any halo. Here we have defined $\tilde{r}=r / r_{s}$ and $\tilde{v}=v / v_{s}$, with $v_{s}=\sqrt{G \rho_{s} r_{s}^{2}}$. $\tilde{f}(\tilde{\boldsymbol{x}}, \tilde{\boldsymbol{v}})$ can be extracted numerically as a template distribution function. Once this is done, the full distribution function is known for any halo parameters, and can be fit against data to obtain best-fit halo parameters. Note the universality of this distribution function over a wide range of halo parameters remain to be explicitly tested. However, it is believed that the universality should at least hold locally over a small range of halo parameters.

In 2008, Wojtak et al. [407] first studied such a rescaled distribution function of dark matter particles in simulated cluster halos. Instead of working in $(\boldsymbol{r}, \boldsymbol{v})$ space, they study the distribution function as a function of the rescaled energy, $\tilde{E}=E / v_{s}^{2}$, and rescaled angular momentum, $\tilde{L}=L / r_{s} v_{s}$, because Jeans theorem implies the distribution function can be expressed as functions of these integrals of motion.

In 2017, Li et al. [234] first exploited the idea of using a template distribution function for satellite galaxies to infer the MW halo mass. In this pioneering work, the template is also built in energy and angular momentum space. However, instead of using a full distribution function, they built their template as well as the likelihood function using the distribution of energy and angular momentum parameters, $p(E, L)=\mathrm{d}^{2} P / \mathrm{d} E \mathrm{~d} L$. As $E$ and $L$ are not direct observables, the likelihood function in $(E, L)$ space leads to a biased halo mass estimator. In addition, their distribution function is parametrized with a single halo mass parameter instead of two NFW parameters, so that the halo concentration cannot be constrained. A halo-to-halo scatter is also found beside the overall bias mentioned before, which is attributed to the variation in the distribution function due to different halo formation histories.

Callingham et al. in 2019 [72] adapted the Li et al. [234] method and applied it to classical satellites in our MW. MWlike galaxies selected from the cosmological hydrodynamical 
EAGLE simulation [83, 338] are used to build a template distribution function and to calibrate the estimator bias. An independent set of halos from the AURIGA simulations are used to further test the method and the bias calibration. Applied to the observed classical satellites, the virial mass of our MW was found to be $M_{200}=1.17_{-0.15}^{+0.21} \times 10^{12} \mathrm{M}_{\odot}$. Combined with independent measurements from other studies, which provided the enclosed mass within a smaller radius, the halo concentration was estimated as $c_{200}=10.9_{-2.0}^{+2.6}$.

Very recently, Li et al. in 2019 [236] improved their method published in 2017 [234]. Starting from a distribution of orbital parameters, the complete probability distribution function in $(r, E, L)$ space can be derived as

$p(r, E, L)=p(r \mid E, L) p(E, L)$.

The second part of this distribution can be obtained from the template distribution function, $p(E, L)=$ $p(\tilde{E}, \tilde{L}) \mathrm{d} \tilde{E} \mathrm{~d} \tilde{L} / \mathrm{d} E \mathrm{~d} L$. For a steady-state distribution function, the distribution along each orbit, $p(r \mid E, L)=\mathrm{d} P(r \mid E, L) / \mathrm{d} r$ is given by Equation (29) (see section 5.4). The distribution function in $(\boldsymbol{r}, \boldsymbol{v})$ space is related to $p(r, E, L)$ by a coordinate transformation,

$p(\boldsymbol{r}, \boldsymbol{v})=\frac{\left|v_{r}\right|}{8 \pi^{2} L} p(r, E, L)$,

where $p(\boldsymbol{r}, \boldsymbol{v})=f(\boldsymbol{r}, \boldsymbol{v}) / N_{\text {tot }}$ is the normalized (or probability) distribution function.

Once the model distribution function given by Equation (28) is obtained, a likelihood estimator can be constructed given the observed $(\boldsymbol{r}, \boldsymbol{v})$ of each tracer. This is a proper likelihood to use compared to those used in [234] and [72], and thus free from the systematic bias due to improper likelihood function. In a follow-up paper, Li et al. [235] applied this new estimator to a sample of 28 satellite galaxies between 40 and $300 \mathrm{kpc}$, with proper motions taken from GAIA DR2. Using a template distribution extracted from the EAGLE simulation, the Milky Way halo mass was best constrained to be $M_{200}=1.23_{-0.18}^{+0.21} \times 10^{12} \mathrm{M}_{\odot}$, and the concentration was constrained as $c_{200}=9.4_{-2.1}^{+2.8}$. Combined with the rotation curve measured by halo stars, tighter constraints were given as $M_{200}=1.26_{-0.15}^{+0.17} \times 10^{12} \mathrm{M}_{\odot}$ and $c_{200}=10.4_{-1.9}^{+2.3}$. Using multiple tracer populations is thus very helpful to better infer the halo concentration. Dependencies on the adopted templates are also discussed in [235].

\subsection{Free-form distribution functions}

In this subsection, we briefly introduce a set of methods with more general assumptions about the distributions. These methods generally do not assume a fixed functional form of the distribution function, but rather allow for a very flexible distribution function to be constrained by the data itself.

The starting point of these methods is the steady-state assumption. If a system is in a steady-state, then phase-space continuity (i.e., the collisionless Boltzmann equation) implies that the distribution of particles along each orbit is determined by the travel time distribution on the orbit [171], i.e.,

$\mathrm{d} P(x \mid$ orbit $) \propto \mathrm{d} t(x)$

Han et al. in 2016 [171] also explicitly showed that the above equation is equivalent to the Jeans theorem. With this conditional distribution along each orbit, the construction of a full distribution function still needs to specify the distribution of orbits, which can be constrained by the data itself while fitting for the underlying potential.

The most classical method of this family is perhaps the Schwarzschild method [342, 429], which works by parameterizing the distribution of orbits with histograms in orbit space. The number of orbits at each grid point in the orbital parameter space (or the orbit library), is left as a free parameter. For each orbit, the distribution along the orbit can be computed by Equation (29) once a potential model is assumed. These combined then predict the phase-space distribution, which can be compared against the observed distributions to solve for the distribution of orbits as well as the best-fit potential. As this method numerically builds a distribution function that is binned in orbit space, it can work for any potential and for incomplete phase-space data. On the other hand, as the number of parameters (including the gridded orbit counts) is large and the orbits need to be integrated numerically, this method is usually computationally expensive.

In order to build smoothly varying histograms of orbits, Bovy et al. (2010) [48] proposed to model the histogram with a Gaussian process with some hyper parameters that are further marginalized during the inference. They applied their method to infer the potential of the Solar system using planets as tracers. Magorrian in 2014 [250] proposed to model the distribution of orbits with an arbitrary number of Gaussians in action space, and then marginalizing over the proposed prior distribution of the parameters of the Gaussians. Because some assumptions on the form of the distribution and on the priors are still needed, these methods are still not fully assumption free. They exist as a trade-off among model flexibility, model smoothness and computational efficiency.

In fact, if the full phase-space coordinates of particles are available, the distribution of orbits can be specified by the data itself instead of being proposed with a library. This is because once a model potential is assumed, the observed phase- 
space coordinates of each particle then fully specify its orbital parameters. This is essentially the key difference between the orbital Probability Density Function (oPDF) method proposed in Han et al. in 2016 [171] and the Schwarzschild method. The use of the data-inferred distribution of orbits in place of a gridded library significantly simplifies the inference of the potential, at the cost of losing the flexibility to handle missing dimensions in the data. This method has been used to study the dynamical state of simulated MW halos in $[172,173,390]$. Their analyses have revealed a stochastic scatter in the best-fit mass and concentration parameters, which can be as large as a factor of 2 when halo stars are used as tracers. These have been interpreted as being caused by phase-correlations in the tracer particles, which violate the steady-state assumption of the model. We give more discussions on such biases in section 10.2. Note that this stochastic bias undermines the precision of any steady-state method. As [391] explicitly demonstrated, the SJE, which is a completely different steady-state method, exhibits a very similar bias when tested on the same set of simulated halos. While the stochastic bias using stars is large, the bias using dark matter particles is much smaller, $\sigma_{M} \sim 20 \%$. It is also shown that satellite galaxies have a dynamical state close to dark matter particles, and are thus expected to be better dynamical tracers than halo stars [173].

When the spatial coordinate of particles are specified by the action angles, Equation (29) translates to a uniform distribution in angle, as the angles evolve uniformly in time. For each assumed potential, one can convert the spatial coordinates of each particle to action angles. The true potential can be found as one that reproduces a uniform angle distribution. In practice, this is achieved by minimizing some distances between the converted and the expected distributions. Beloborodov and Levin in 2004 [13] first proposed two such minimum distance estimators. However, as shown in [171], these estimators are usually less efficient than likelihood estimators such as the oPDF and suffer from strong degeneracies between the halo mass and concentration parameters.

In the end, we note that none of the above methods in this sub-section have been applied to real data of the MW.

\section{Modeling the stripping and evolution of tidal debries: stellar streams}

As mentioned in Sec. 1, stellar streams are formed by stripped stars from satellite galaxies or from globular clusters through tidal forces. These tidally formed stellar streams (tidal streams or debries) contain a wealth of information on structure formation, galaxy evolution, dynamics of progenitor satellites and the underlying potential.
Early studies of tidal debris in our MW and nearby galaxies used photometric plates [e.g. 166, 228, 229, 230]. However, tidal streams in the MW can extend tens of degrees over the sky, and thus surveys covering large areas are crucial for detecting them. Nowadays, deep and large sky surveys have enabled detections of tidal streams in both our MW and nearby galaxies. For example, the Sloan Digital Sky Survey [415, SDSS] has enabled a numerous number of detections of tidal streams in our MW, which are either associated with known globular clusters, satellite galaxies or without obvious associations [e.g. 7, 15, 16, 33, 160, 161, 162, 163, 164, 165, $167,285,286]$.

More and more imaging surveys have added to the growing list of detected tidal structures in our MW, including the study of Koposov et al. in 2014 [216] using the VST ATLAS survey [347], the findings by Martin et al. in 2014 [255] based on the Pan-Andromeda Archaelogical Survey [260, PAndAS], Grillmair et al. in 2013 [163] using data from the 2MASS Point Source Catalog [355] and the Wide Field Infrared Survey Explorer (WISE) All-Sky Release [408], Bernard et al. in 2014 [20] based on the Pan-STARRS1 [PS1; 201] 3 $\pi$ survey, Shipp et al. in 2018 [350] with the Dark Energy Survey [DES; 321], and some other studies [e.g. 274, 367]. Streams and tidal features are also commonly detected in nearby galaxies thanks to deep photometry [e.g. 196, 256, 371].

Almost all of the above detections were made from photometric data, and only few combined radial velocities from spectrocopic data and proper motions. With available velocity information, in fact some tidal streams were either solely or partly detected in velocity space $[75,180,207,271,281$, $344,362,405]$. There were also efforts of looking for debris and substructures in action space [e.g. 272, 273] or through machine learning approaches [e.g. 418, 419].

With on-going and up-coming spectroscopic surveys (see those mentioned in the introduction), increased proper motion data from Gaia, and even deeper imaging surveys in the future such as the Large Synoptic Survey Telescope [LSST; 192], we expect growing observations of tidal debris and increasing kinematical data of resolved stars with 3-dimensional velocities associated to tidal streams. Because tidal streams can extend over large distances, their dynamics are sensitive to both the depth and shape of the Galactic gravitational potential [e.g. 45, 178, 195, 246, 257], and it was proposed that dark matter substructures can induce localized fluctuations and gaps along such long streams, which can be used to detect dark matter subhalos and dark streams [e.g. 35, 43, 46, 74, 119, 334, 414]. Despite the richness of data and the valuable dynamical information, most of the studies on tidal streams were theoretical [e.g. 43, 128, 129, 381], or qualitative and empirical $[17,132,246]$, or based on nu- 
merical simulations without contaminations and errors [e.g. 34, 36, 98, 225, 268, 317, 335].

A number of methods have been developed to model observed tidal streams. These include orbit fitting [e.g. $217,280,384]$ to the tidal stream and the remnant of the progenitor, if the progenitor still survives and the association can be identified, N-body simulations [105, 226, 227], approaches of particle releasing/spraying [34, 148, 222, 223], semi-analytic approaches $[104,105]$ and action angle distribution of tidal debries [e.g. 41, 180, 327, 329]. Relatively fewer studies had specifically constrained the mass of our MW, among which only two measurements are selected into Fig. 1 that provided virial mass estimates with statistical errors.

Early in 1995, Lin et al. [238] made orbit modeling of the observed distances and motions of the Magellanic clouds and segments along the Magellanic stream ${ }^{9)}$, and through their modeling the mass within $100 \mathrm{kpc}$ of our MW was estimated to be $5.5 \pm 1.0 \times 10^{11} \mathrm{M}_{\odot}$.

Koposov et al. in 2010 [217] made orbit fitting to the 6dimensional phase-space map of the thin but extended ( 60 degrees) GD-1 stream, and placed strong constraints on the local circular velocity $(221 \pm 18 \mathrm{~km} / \mathrm{s})$ at the solar orbital radius. More recently, with new data from Gaia, SEGUE and LAMOST for the GD-1 stream, Malhan and Ibata in 2019 [251] constrained the local circular velocity to be $244 \pm 4 \mathrm{~km} / \mathrm{s}$, and the mass within $20 \mathrm{kpc}$ to the Galactic center was estimated as $2.5 \pm 0.2 \times 10^{11} \mathrm{M}_{\odot}$.

Through orbit fitting to BHB stars in the so-called "Orphan stream" discovered by Grillmair [160] and Belokurov et al. [17] in 2006, which spans about 60 degree over the sky, the total mass within $60 \mathrm{kpc}$ of our MW was estimated to be $\sim 2.7 \times 10^{11} \mathrm{M}_{\odot}$ by Newberg et al. in 2010 [280]. The mass out to $240 \mathrm{kpc}$ was extrapolated to be $\sim 6.9 \times 10^{11} \mathrm{M}_{\odot}$ assuming a $\log$ potential. More recently, Hendel et al. in 2018 [181] conducted orbit fitting to RR Lyrae stars in the Orphan stream, and an upper limit on the MW mass enclosed within $60 \mathrm{kpc}$ was constrained to be $5.6_{-1.1}^{+1.2} \times 10^{11} \mathrm{M}_{\odot}$.

It is often assumed that the orbit of the progenitor is traced by the stream and the motions of stripped stars are all aligned with the stream track. The assumptions might not be strictly valid [e.g. 129, 215]. In addition, it was pointed out by Lux et al. [247] and by Sanders and Binney [328] in 2013 that a single orbit fitting to the observed dynamics of a tidal stream may lead to significant biases. Thus, realistic modelings of not only the orbit of the progenitor, but how stars along the stream are stripped and evolved are necessary.

$\mathrm{N}$-body simulations are powerful tools to model and un- derstand the formation histories of tidal streams and their progenitors. Comparing $\mathrm{M}$ giant stars along the Sagittarius stream and with N-body simulations and test particle orbits, Law et al. in 2005 [226] constrained the total mass within $50 \mathrm{kpc}$ of our MW to be in the range of $\sim 3.8$ to $5.6 \times 10^{11} \mathrm{M}_{\odot}$. However, with $\mathrm{N}$-body simulations, it is very expensive to properly explore the parameter space and obtain a best-fit model potential with a robust confidence region. As a result, the number of studies relying on N-body simulations to explore the parameter space is very limited at the current stage.

Gibbons et al. in 2014 [148], Bowden et al. in 2015 [52] and Küpper et al. in 2015 [222] subsequently proposed less expensive approaches of generating tidal streams, which involves steadily releasing particles through the two Lagrangian points of the progenitor and evolving the released particles within given potential models. The approach is less expensive compared with standard N-body simulations. The one proposed by Küpper et al. in 2015 [222] was called the STREAKLINE. The initial velocities of particles can be modeled through the velocity of the progenitor, modulated to match the instantaneous angular velocity of the object center with respect to the galactic center, plus some scatters [34, 223]. Particles released from the two Lagrangian points formed the leading and trailing arms of the stream. In particular, Küpper et al. [222] chose to ignore the scatter and fit the coldest model stream to observed density peaks along the Palomar 5 stream, with its trailing stream extending 23.2 degrees and leading arm cut off by the survey edge. The virial mass was found to be $M_{200}=1.69 \pm 0.42 \times 10^{12} \mathrm{M}_{\odot}$. Within the apocenter of Palomar 5 ( $\sim 19 \mathrm{kpc})$, the enclosed mass of the Galaxy (disk+bulge) was estimated to be $2.14_{-0.35}^{+0.38} \times 10^{11} \mathrm{M}_{\odot}$. The circular velocity at the solar radius was constrained as $253 \pm 16 \mathrm{~km} / \mathrm{s}$.

The method proposed by Gibbons et al. in 2014 [148] also relied on releasing particles through the Lagrangian points and they applied their method to constrain the mass of our MW as well. They in addition considered the progenitor's gravity, which was shown to be very crucial in order to bring consistency with direct N-body simulations. Applying the method to the famous Sagittarius stream, the total mass within $100 \mathrm{kpc}$ was constrained to be $4.1 \pm 0.7 \times 10^{11} \mathrm{M}_{\odot}$. The mass was extrapolated to $200 \mathrm{kpc}$ as $0.56 \pm 0.12 \times 10^{12} \mathrm{M}_{\odot}$, i.e., a "light" MW.

Recently, it was reported that the stellar motions in the Orphan stream are misaligned with the stream track[215]. Based on the Gibbons et al. 2014 method, Erkal et al. in 2019 [120] found the motion-track misalignment can be well explained by the LMC perturbation to the MW potential.

9) Later studies based on proper motions of Magellanic Clouds have reported that the stream might be formed through local interactions between large and small Magellanic Clouds. See Sec. 8.1 for details. 
Jointly fitting an LMC mass and a MW potential, the MW mass within $50 \mathrm{kpc}$ was estimated to be $3.8_{-0.11}^{+0.14} \times 10^{11} \mathrm{M}_{\odot}$.

Dierickx et al. in 2017 [104] considered tidal stripping and dynamical friction in their modeling of the progenitor, following a semi-analytical approach. Their approach was based on orbit fitting to the satellite remnant and the Sagittarius stream, but instead of integrating the current position and velocity of the progenitor back in time, Dierickx et al. [104] modeled the progenitor forward in time and tried a series of initial velocities and orbit angles for different masses of the MW and progenitor masses of the stream. It was found that massive MW halos had difficulties to reproduce the velocity and distance of the progenitor simultaneously, resulting in an upper limit to the virial mass of our MW as $10^{12} \mathrm{M}_{\odot}$.

\section{The timing argument and local Hubble flow: the motion of MW and M31}

In this section we will introduce how to constrain the mass of our MW and the Local Group by modeling the relative motion of MW and M31, and the motion of distant satellite galaxies in our Local Group. Measurements in this section fall in the category of "timing \& LG dyn." in Fig. 1.

\subsection{Timing argument}

M31 is the massive companion of our MW Galaxy. Currently MW and M31 are approaching each other. Our Universe is expanding, but gravitational forces can reverse the expansion locally. In our Local Group (hereafter LG), MW and M31 are the two dominating galaxies, and their distances to the nearest external bright galaxy are much larger than the separation between themselves. The fact that they are approaching each other can thus be used as constraints of the mass associated with them.

Early in 1995, Kahn and Woltjer [200] pointed out that galaxies were at zero separation at the Big Bang, and thus they must have passed through apocenters at least once in order to be approaching each other today. This requires that the apocentric distance of the orbit must be larger than the current separation between them, and half of the ortibal period should be smaller than the age of the Universe. These requirements help to provide a lower limit on the total mass of our MW and M31.

There are evidences that the tangential velocity of M31 with respect to our MW is negligible. Ignoring the tangential velocity and cosmic expansion and further assuming point mass, the equation of energy conservation along the orbit can be written as

$\frac{1}{2}\left(\frac{\mathrm{d} r}{\mathrm{~d} t}\right)^{2}-\frac{G M}{r}=-\frac{1}{2} \frac{G M}{a}$,

where $M$ is the total mass of MW and M31, $r$ is the separation, $\frac{\mathrm{d} r}{\mathrm{~d} t}$ is the relative velocity, $a=G M /(-2 E)$ and $2 a$ is the maximum value of $r$ on the orbit, $E$ is the orbital energy.

The solution to the separation and velocity can be obtained by introducing an angle-like quantity of $\eta$, which is referred as the eccentric anomaly

$$
\begin{aligned}
& \text { (i) }: r=a(1-\cos 2 \eta), \\
& \text { (ii) }: \frac{\mathrm{d} r}{\mathrm{~d} t}=\sqrt{\frac{G M}{a}} \frac{\sin 2 \eta}{1-\cos 2 \eta}
\end{aligned}
$$

$\eta$ is related to time, $t$, through the following equation and can be solved numerically

$\eta-\sin 2 \eta=\left(\frac{G M}{a^{3}}\right)^{1 / 2} 2 t$.

Equation 31 can be used to constrain the total mass, $M$, for our LG. Given the observed separation and velocities of MW and M31, plus the age of our Universe, the solution to the above equations with a single apocentric passage gives the lowest limit of the total mass. Such a lower limit of the LG mass was measured to be $5 \times 10^{12} \mathrm{M}_{\odot}$ by Kahn and Woltjer in 1995 [200].

More recently, using proper motion data from the multiepoch HST/ACS photometry, van der Marel et al. in 2012 [380] concluded that the tangential component of the M31 velocity with respect to $\mathrm{MW}$ is statistically consistent with being negligible, and the M31 orbit towards MW is radial. They revised the $\mathrm{LG}$ timing mass to be $4.93 \pm 1.63 \times 10^{12} \mathrm{M}_{\odot}$.

Similar approaches can be adopted to constrain our MW mass, by considering the system formed by our MW and distant dwarf satellite galaxies. The estimated total mass mainly reflects the mass of our MW, as satellites are sub-dominant. Based on the MW-Leo I system and assuming radial orbits, Zaritsky et al. in 1989 [422] derived a lower mass limit of $1.3 \times 10^{12} \mathrm{M}_{\odot}$ for our MW. More recently, Li and White in 2008 [233] calibrated the MW timing mass, using halo pairs in analogy to MW and Leo I in the cosmological Millennium simulation [366]. Basically, the timing approach were applied to halo pairs in the simulation, and the timing mass can be compared with the true virial mass in the simulation to quantify the bias. The virial mass of our MW was calibrated to be $M_{200}=2.43 \times 10^{12} \mathrm{M}_{\odot}$ with a $95 \%$ lower confidence limit of $0.8 \times 10^{12} \mathrm{M}_{\odot}$, which was at the massive end compared with other contemporary measurements. 
Applying the timing approach to the MW-Leo I system required the boundedness of Leo I. Besides, none-zero tangential velocity of Leo I would increase the timing mass. Using two epochs of HSC WCS/WFC observations spanning a fiveyear time baseline, Sohn et al. in 2013 [361] measured the proper motion of Leo I. The tangential velocity with respect to the Galactic center was estimated to be $\sim 101.0 \pm 34.4 \mathrm{~km} / \mathrm{s}$, which is inconsistent with radial orbits. With the observed radial and tangential velocities of Leo I, [361] concluded the boundedness of Leo I to our MW. Solving the complete and non-radial timing equation, the orbit of Leo I inferred a virial mass of our MW as $M_{200}=2.65_{-1.36}^{+1.58} \mathrm{M}_{\odot}{ }^{10)}$

Instead of modeling a single object, Zaritsky et al. in 2019 [420] applied the timing argument to a sample of 32 stars with Galactocentric distances larger than $60 \mathrm{kpc}$. The timing mass was calibrated and compared with the suite of AURIGA simulations to obtain a statistical estimate of our MW virial mass in the range of $0.91 \times 10^{12} \mathrm{M}_{\odot}<M_{200}<2.13 \times 10^{12} \mathrm{M}_{\odot}$ (90\% confidence).

Benisty et al. in 2019 considered different numbers of past encounters between MW and M31 and tried different gravity models [18] in their estimates of the LG mass under the timing framework, though past encounters do not seem to be supported by recent Gaia data [e.g. 143].

Very recently, Zhai et al. in 2020 [423] looked for MW31 like systems in numerical simulations, and they found that higher tangential velocities correspond to higher total mass and also affect the individual mass distribution of MW and M31 analogs. The typical host halo mass of MW is $1.5_{-0.7}^{+1.4} \times 10^{12} \mathrm{M}_{\odot}$ for radial orbits between $\mathrm{MW}$ and $\mathrm{M} 31$, and $2.5_{-1.4}^{+2.2} \times 10^{12} \mathrm{M}_{\odot}$ for low-ellipticity orbits.

\subsection{The local Hubble flow}

The timing approach can also be applied to model the relative motion of nearby galaxies in the local volume towards the LG. Assuming the companion galaxies are massless, it can help to constrain the LG mass. Under the timing framework, Peñarrubia et al. in 2014 [299] specifically modeled the dynamics of galaxies in the local volume of an expanding universe (Eqn. 33), using published distances and velocities of nearby galaxies within $3 \mathrm{Mpc}$ where the gravitational force reverses the expansion

$\frac{\mathrm{d}^{2} r}{\mathrm{~d} t^{2}}=-\frac{G M}{r^{2}}+H_{0}^{2} \Omega_{\Lambda} r$.

$\mathrm{M}$ is the total mass of LG. $r$ is defined with respect to the

\section{LG barycenter.}

Basically, given the LG barycenter (or mass ratio between MW and M31) and the circular velocity at the solar radius, which were treated as model parameters, the distance $\left(r\left(t_{0}\right)\right)$ and radial velocity $\left(V\left(t_{0}\right)\right)$ of an observed galaxy to the $\mathrm{LG}$ barycenter at current epoch $t_{0}$ can be calculated. Then after choosing a small initial radius of the galaxy, its initial velocity can be solved through Eqn. 33, by requiring that the integrated distance at time $t_{0}$ agrees with the distance, $r\left(t_{0}\right)$, calculated in the previous step. Note $H_{0}, \Omega_{\Lambda}$ and $M$ are model parameters in this step. In the end, the integrated radial velocity of the galaxy at $t_{0}$ can be compared with $V\left(t_{0}\right)$ through the likelihood function, in order to find the set of best-fit parameters for the circular velocity at the solar radius, the LG barycenter, the LG mass, the cosmological constant and the Hubble constant. [299] derived the LG mass to be $2.3 \pm 0.7 \times 10^{12} \mathrm{M}_{\odot}$ and a mass ratio between $\mathrm{MW}$ and $\mathrm{M} 31$ as $0.54_{-0.17}^{+0.23}$. Hence the virial mass of our MW was estimated as $0.8_{-0.3}^{+0.4} \times 10^{12} \mathrm{M}_{\odot}$. In their analysis, both the LG quadrupole and the time variance of the potential were considered and discussed, which had negligible effects to the results.

In two follow-up papers, Peñarrubia et al. in 2016 [298] and Peñarrubia and Fattahi in 2017, [297]), the effects of the LMC and the fraction of mass outside the virial radius that perturbs the local Hubble flow were taken into considerations. The LMC can change the barycenter velocity of nearby galaxies and lead to an updated virial mass of our MW as $1.04_{-0.23}^{+0.26} \times 10^{12} \mathrm{M}_{\odot}$. Moreover, using a set of hydrodynamical simulations of MW-like halos and galaxies from the APOSTLE project [A Project of Simulations of The Local Environment; 131, 337], it was found that a relatively large fraction of the mass perturbing the local Hubble flow and driving the relative trajectory of the main galaxies is not contained within the halo virial radius. Adopting the outer halo profiles in $\mathrm{N}$ body simulations to calibrate the virial mass, it was reported that the mass given by [298] should be divided by a factor of 1.2 , to give the actual mass within the virial radius, which led to the constraint of $M_{200}=\sim 0.87 \times 10^{12} \mathrm{M}_{\odot}$.

\subsection{Momentum of MW and M31}

If the LG is sufficiently isolated from nearby galaxy groups and matter distributions, plus the assumption that the LG mass is dominated by MW and M31, the total momentum of MW and M31 should be close to zero in the rest frame of the LG. If the velocity vector of MW and M31 with respect to the LG barycenter can be measured, the mass ratio between

10) We have converted the original virial mass provided in the paper to $M_{200}$ by dividing a factor of 1.19 , which is the value provided in their paper, based on the NFW halo profile with concentration of 9.5. Not only observational errors, but also the cosmic scatter are included in the errors. [361] calculated the cosmic scatter based on a similar catalog used by the earlier study of Li and White [233]. Note the virial mass was calibrated by [361] against subhalos in numerical simulations having similar tangential velocities to Leo I as well. 
MW and M31 can then be further constrained [103]. Compared with the timing argument, the orbit does not have to be assumed as radial Keplerian orbits. The velocity of MW and M31 with respect to the barycenter of the LG can be decomposed into two components, the Heliocentric velocity of MW (or M31) and the solar motion with respect to the LG barycenter.

The solar motion, as have been described in Sec. 3, is a combination of the velocity of the LSR and solar peculiar velocity with respect to the LSR, and can be measured through the apparent motion of Sgr A* or through modeling the distances and velocities of maser sources. The Heliocentric motion of MW is simply the solar motion added with a negative sign. The Heliocentric velocity of M31 has be measured through spectroscopic observations for the line-of-sight component [259], and its proper motion has been measured through high-precision astrometry of HST [360].

The solar motion with respect to the LG barycenter can be constrained through observations of distant satellite galaxies with a Bayesian approach. Basically, the radial velocities of distant satellites are the observed Heliocentric velocities plus the solar motion with respect to the LG barycenter. These radial velocities can be assumed to follow a Gaussian distribution such that galaxies within the LG move randomly with uncorrelated motions. This can be used to construct the likelihood and constrain the solar motion with respect to the LG barycenter.

Based on the ideas above, Diaz et al. in 2014 [103] estimated the mass ratio between M31 and MW as $10^{0.36 \pm 0.29}$. Combined with the virial theorem, the total mass of LG was estimated as $2.5 \pm 0.4 \times 10^{12} \mathrm{M}_{\odot}$, and hence the mass of MW was constrained as $0.8 \pm 0.5 \times 10^{12} \mathrm{M}_{\odot}$.

\section{Satellite phenomenology: matching ob- served satellites to simulations}

The population of satellite galaxies in our MW offer various approaches to measure the virial mass of our MW. We have already introduced the example of measuring the MW timing mass based on the MW-Leo I pair (see Sec. 7 for details) with calibrations against numerical simulations. MW satellite galaxies have also been used as dynamical tracers together with globular clusters and halo stars in the SJE modeling and distribution functions (see Sec. 4 and Sec. 5), though whether satellites are in dynamical equilibrium awaits further checks. In this section, we introduce the efforts which constrain the virial mass of our MW by comparing observed bright dwarf spheroidal satellite galaxies in our MW and subhalos in numerical simulations. Some of the studies attempted to select subhalos in simulations that are analogous to observed
MW satellites, and sample the simulated systems under the Bayesian framework to obtain the most likely virial mass for our MW [53, 156, 291, 292]. Some studies simply looked at the fraction of MW satellite-like systems as a function of the virial mass of the host halo in simulations $[9,79]$. There are also attempts which relied on empirical relations derived from simulations that link observed satellite properties to the virial mass of the host halo [325]. The group of measurements introduced in this section fall in the category of "Satellite Phenomen" in Fig. 1.

Such comparisons and calibrations stem on the fact that satellite galaxies can be directly linked to subhalos in $\mathrm{N}$-body or hydrodynamical simulations. This is an advantage compared with stars and globular clusters, as modern hydrodynamical simulations do not have enough power to resolve individual stars, whereas particle painting/tagging approaches have to rely on semi-analytical modeling of stellar evolution and phase-space sampling [e.g. 243].

To properly link observed satellites to simulated subhalos, available proper motions are crucial. With high precision astrometric instruments and imaging data taken at different epochs, accurate proper motions for about ten classical MW satellite galaxies and the Magellanic Clouds had already been measured [e.g. 106, 107, 108, 203, 204, 303, 304, 305, 306, 307, 308, 309, 310, 361]. The classical satellites are bright spheroidal dwarfs, including Leo I and the Sagittarius dwarf introduced in Sec. 2, Sec. 6 and Sec. 7. These satellites have already been used as dynamical tracers in many previous studies based on the SJE, distribution functions or tidal streams. Recently, the proper motions of 9 classical dwarf spheroids, the ultra faint satellite galaxy, Bootes I, and the Magellanic Clouds in our MW were either refined or further measured based on Gaia DR2 [e.g. 142].

\subsection{Magellanic Clouds}

Among the satellite galaxies of our MW, the Large and Small Magellanic Clouds (LMC and SMC) are of great interests. They are very likely accreted by our MW as a group given the similarity in their orbits [203], which is in good consistency with simulation results [e.g. 109].

For galaxies with LMC stellar mass, the typical host halo mass is $\sim 2 \times 10^{11} \mathrm{M}_{\odot}$ before being accreted by a more massive host halo and becoming satellites. The host halo mass of SMC is approximately a factor of 2 to 3 smaller. Interestingly, LMC stellar mass galaxies with an SMC mass satellite are very rare and are typically $\sim 50 \%$ more massive than LMC sized objects, which suggest that the LMC have been as massive as $\sim 3 \times 10^{11} \mathrm{M}_{\odot}$ at infall [78, 298, 348]. Hence the MW's two brightest satellites are massive objects which contribute a considerable fraction of the total MW mass. Such massive 
satellites are rare in halos smaller than $M_{200} \sim 10^{12} \mathrm{M}_{\odot}$ in numerical simulations, but are more common if the host halo is more massive than $M_{200} \sim 2.0 \times 10^{12} \mathrm{M}_{\odot}$ (see discussions in Boylan-Kolchin et al. in 2010 [56]). The probability for such massive satellites to be within MW-like host galaxies as predicted by numerical simulations [53, 70, 291, 348] is in very good agreement with other extra-Galactic MW-like galaxies in SDSS [240, 374].

Before the three-dimensional velocities of MCs were actually measured, it was conventionally believed that MCs have accomplished multiple passages orbiting the MW [e.g. 146, 270]. The argument was motivated by the existence of the long and coherent Magellanic stream, which is a young stream of HI gas spanning $150^{\circ}$ along the sky and was believed to be formed by tidal forces. However, with the measured proper motion, Kallivayalil et al. in 2006 [204] updated the total velocity of the LMC to be $\sim 380 \mathrm{~km} / \mathrm{s}$, larger than the commonly assumed velocity in old studies. In addition, it was found that the observed three-dimensional velocities of the LMC were not aligned with the Magellanic stream. Thus the Magellanic stream might have formed through local interactions between the LMC and SMC, rather than formed by the MW's tidal or ram-pressure stripping (see the paper by Besla et al. in 2010 [22]). Follow-up papers based on the measured high velocity of the massive LMC argued that the LMC was very likely accreted late and on its first passage near the orbit pericenter [21, 53, 69, 156, 293]. The time it spends close to the orbit pericenter is short due to its high speed, which might explain why LMC analogues in numerical simulations matched in Galactocentric distances and velocities are rare.

Looking for subhalos which have similar masses and velocities as that of the LMC in the cosmological MillenniumII simulation [MRII; 57], Boylan-Kolchin et al. in 2011 [53] claimed that the virial mass of our MW is unlikely smaller than $M_{200} \sim 1.25 \times 10^{12} \mathrm{M}_{\odot}$. A similar conclusion was reached by Patel et al. in 2017 [293] based on the high resolution and dark matter only run of the cosmological Illustris simulation [147, 278, 385, 386]. The orbital energies of LMC analogues in the Illustris simulation favor a MW halo mass of $1.5 \times 10^{12} \mathrm{M}_{\odot}$.

Tighter constraints on the mass of our MW have been subsequently made by Busha et al. in 2011 [69], González et al. in 2013 [156] and Patel et al. in 2017 [291], by comparing the observed positions, velocities and masses of MCs with $\mathrm{MC}$-analogues in numerical simulations under the Bayesian framework. Based on dark matter halos in the cosmological Bolshoi simulation [211], Busha et al. in 2011 [69] statistically sampled subhalos in a large population of host dark matter halos. The observed Galactocentric distances, total velocities and the circular velocities of both the LMC and
SMC were used to construct the likelihood that a halo of a given mass can host two satellites with these properties, and the prior was represented by the sample of halos in the simulation. The posterior probability distribution function was calculated through importance sampling. The virial mass of our MW was constrained to be $M_{200}=1.0_{-0.4}^{+0.7} \times 10^{12} \mathrm{M}_{\odot}$.

González et al. in 2013 [156] further investigated the effect of the LG environment on estimating the virial mass of our MW. It was found that satellites in host halos of LGlike environments tend to have slightly larger velocities, but it does not significantly affect the likelihood. González et al. [156] derived the virial mass of our MW to be $M_{200}=$ $1.15_{-0.34}^{+0.48} \times 10^{12} \mathrm{M}_{\odot}$, which is in good agreement with the earlier measurement by Busha et al. [69].

Based on the dark matter only run of the Illustris simulation, Patel et al. in 2017 [291] have selected satellite galaxies in host halos with different virial masses, using the observed Galactocentric distance, total velocity, circular velocity and the specific angular momentum of the LMC (SMC was not used in their analysis), and have employed a similar Bayesian analysis as Busha et al. [69]. Patel et al. have found that the specific angular momentum of satellites is well conserved, and the virial mass of our MW is $M_{200}=0.83_{-0.55}^{+0.77} \times 10^{12} \mathrm{M}_{\odot}$. In a later study, Patel et al. in 2018 [292] extended the method to all MW satellites with available proper motions. They found that the scatter among mass estimates based on individual satellite can be reduced by using the specific angular momentums instead of a satellite's velocity. Joint constraints based on all classical satellites suggested a virial mass of $0.68_{-0.26}^{+0.23} \times 10^{12} \mathrm{M}_{\odot}$. If one were to exclude the Sagittarius dwarf satellite, the measured virial mass would be $0.78_{-0.28}^{+0.29} \times 10^{12} \mathrm{M}_{\odot}$.

\section{2 $V_{\max }$ distributions, orbital ellipticities and velocity dispersions of classical satellite galaxies}

In addition to $\mathrm{MCs}$, there are about 10 classical dwarf spheroidal satellites in our MW. Early attempts of using these classical satellites to constrain the MW virial mass involved using the velocity dispersion of the population. Sales et al. in 2007 [325] analyzed subhalos and satellite galaxies in a suite of N-body and hydrodynamical simulations. They found that the spatial and kinematic distributions of satellites trace well that of dark matter, and that the velocity dispersion of the satellites is closely related to the virial velocity of the host halo, $\sigma_{\text {sat }} / V_{\text {vir }} \sim 0.9 \pm 0.2$. Applying the relation to the velocity dispersion of MW classical satellites, the virial velocity of the MW was constrained to be $109 \pm 22 \mathrm{~km} / \mathrm{s}$. This corresponds to a very low MW virial mass of $M_{200}=0.58_{-0.20}^{+0.24} \mathrm{M}_{\odot}$.

As mentioned above, MCs are massive, whose maximum circular velocities, $V_{\max }$, are greater than $60 \mathrm{~km} / \mathrm{s}$. On the 
other hand, most classical satellites in our MW have $V_{\max }$ smaller than $30 \mathrm{~km} / \mathrm{s}$, except for the Sagittarius dwarf. So basically, at most three satellite galaxies of our MW (the LMC, SMC and potentially the Sagittarius dwarf) have $V_{\max } \geqslant$ $30 \mathrm{~km} / \mathrm{s}$, whereas all the other satellites have $V_{\max }<30 \mathrm{~km} / \mathrm{s}$. There is an apparent lack of objects with $V_{\max }$ between 30 and $60 \mathrm{~km} / \mathrm{s}$, which leads to the so-called "too big to fail" problem that our MW does not have enough massive satellite galaxies (only three) to match the number of massive subhalos in numerical simulations. The problem can be resolved if the virial mass of our MW becomes smaller than $1 \times 10^{12} \mathrm{M}_{\odot}$ $[80,388]$, but as have been introduced in the previous section, the existence of LMC and SMC in $\sim 10^{12} \mathrm{M}_{\odot}$ halos is rare.

Using the cosmological MillenniumII simulation, Cautun et al. in 2014 [79] found that the virial mass of our MW should satisfy $M_{200} \leqslant 1.4 \times 10^{12} \mathrm{M}_{\odot}$ to meet the condition of only three satellites with $V_{\max } \geqslant 30 \mathrm{~km} / \mathrm{s}$, whereas the condition of hosting LMC and SMC-like subhalos requires $M_{200}>1 \times 10^{12} \mathrm{M}_{\odot}$. Combining the two requirements, the most plausible virial mass for a halo to host a MW-like population of subhalos was estimated to be $M_{200}=$ $0.78_{-0.33}^{+0.57} \times 10^{12} \mathrm{M}_{\odot}$. The confidence region is given by the fraction of halos in the simulation which have at most three subhalos with $V_{\max } \geqslant 30 \mathrm{~km} / \mathrm{s}$, and at least two subhalos with $V_{\text {max }} \geqslant 60 \mathrm{~km} / \mathrm{s}$.

Based on MW-like halos in the Aquarius simulation [365], Barber et al. in 2014 [9] investigated the orbital ellipticity distribution of subhalos. They have found that the orbital ellipticity distribution of subhalos which can plausibly host luminous satellites show little halo-to-halo variations in cosmological simulations. Given a set of fiducial MW virial masses, Barber et al. [9] inferred the orbit ellipticity of nine galactic classical satellites, which were then compared with the simulation-based orbital ellipticity distribution. The virial mass of our MW was constrained to be $M_{200}=1.10_{-0.29}^{+0.45} \times 10^{12} \mathrm{M}_{\odot}$, in order to bring consistency between observed and simulation based orbit ellipticity distributions.

\section{Other methods}

\subsection{Total mass estimated from baryonic mass fraction}

A dynamics-free lower mass limit has been estimated for our MW by Zaritsky and Courtois in 2017 [421], based on the total baryonic matter and the cosmological baryon fraction. To estimate the total baryonic matter, Zaritsky and Courtois [421] used a sample of MW-like disk galaxies which have measured stellar mass and cold disk gas mass, while the mass confined in hot and cold halo gas was taken from other studies [170, 264, 283, 397]. The total baryonic mass was converted to the projected total mass assuming the baryon fraction in MW-like galaxies is the same as inferred by the cosmological baryon fraction. Based on the mass distribution of their $151 \mathrm{MW}$-like galaxies and the measurement uncertainties of cold and hot gas mass, they estimated a $10 \%$ lower percentile of $7.7 \times 10^{11} \mathrm{M}_{\odot}$ and a median of $1.2 \times 10^{12} \mathrm{M}_{\odot}$.

\section{Summary and discussion}

The last two decades have seen a multitude of determinations of the virial mass of our Galaxy using a diversity of methods and tracer populations. This review is an attempt to summarize and classify the various approaches used in literature, and to highlight potential ways in which future progress can be made. The numerous studies and methods used are best outlined by Fig. 1, which encapsulates the previous determinations of the MW virial mass. It shows that there are no less than 47 individual MW mass measurements using seven broad classes of methods (see Table 1 for a short summary of these classes).

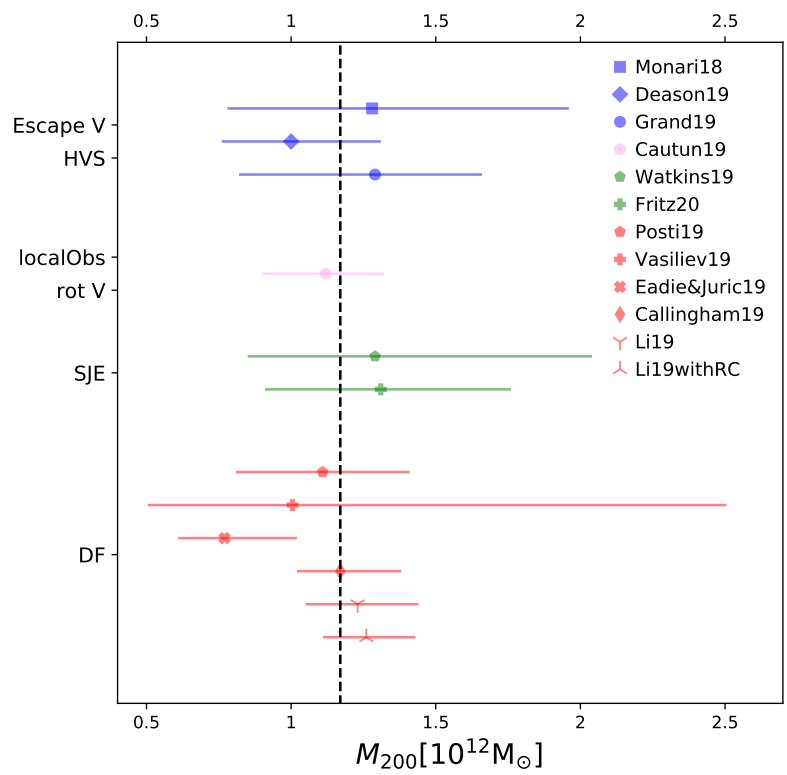

Figure 5 Similar to Fig. 1, but it shows only recent MW mass measurements that made use of the Gaia DR2 data. Such measurements typically use a large number of tracers with full 6D phase-space data and are less affected by systematic uncertainties (see main text). For reference, the vertical dashed line marks the median value among all 11 measurements. Note that this plot shows a narrower $M_{200}$ range than Fig. 1 (it roughly corresponds to the range between the two vertical dotted lines in Fig. 1). The errorbars correspond to $68 \%$ confidence intervals.

Our compilation of total mass measurements highlights that the virial mass of our Galaxy is still uncertain to within at least a factor of two, and that probably its value lies in the interval $[0.5,2.0] \times 10^{12} \mathrm{M}_{\odot}$. The large uncertainty interval 
is a byproduct of two effects. Firstly, some of the first studies in the subject had access to a limited and potentially biased number of observations, typically with large measurement errors, and thus those results have large uncertainties associated to them. Secondly and more worryingly, some measurements have potentially systematic biases that are not taken into account in the quoted error bars. This explains why at least a couple of results (e.g. see some of the distribution function results in Fig. 1) have very small error bars, however those measurements do not overlap within reasonable confidence limits.

We also find systematic differences in the inferred MW virial mass between classes of methods. For example, the determinations based on high velocity stars and also on the assumption that the MW satellites are bound (Sec. 2) typically argue for a heavy MW, with a virial mass higher than $1.25 \times 10^{12} \mathrm{M}_{\odot}$. Similarly, the timing argument (Sec. 7), which provides a lower limit to the virial mass of our MW, often prefers a heavy MW as well. A massive MW is also helpful to explain how come our Galaxy simultaneously hosts two very bright satellite galaxies, the LMC and the SMC. In contrast, other classes of methods, such as the dynamics of MW satellites and those of the LG typically suggest MW virial masses below $1 \times 10^{12} \mathrm{M}_{\odot}$, which, for example, are needed to alleviate the too big to fail problem. The other methods, such as the those based on the spherical Jeans equation or on modeling the distribution function, prefer a mass of about $\sim 1.0 \times 10^{12} \mathrm{M}_{\odot}$, with a roughly equal number of measurements above and below this value.

As we just discussed, many mass measurements, especially early ones, are affected by either small sample size or incomplete phase-space data (e.g. proper motions) that can lead to large statistical and, more importantly, systematic uncertainties. The recent Gaia DR2 data, combined with other complementary observations, offer an exquisite data set with millions of stars and tens of satellite galaxies and globular clusters with full position and velocity information. In Fig. 5, we present the subset of MW total mass measurements that use the Gaia DR2 data. Interestingly, these recent measurements show good agreement with each other and even between different classes of methods, with most results overlapping within their $1-\sigma$ uncertainties. The good agreement between methods and the small uncertainties are at least partly due to the availability of Gaia DR2 proper motions. This means that there is no need to make many of the assumptions employed by earlier studies, which had to cope with incomplete data, and thus these recent estimates are not affected by the systematics arising from these assumptions. Furthermore, many of the methods shown in Fig. 5 have been validated and calibrated against numerical simulations (for de- tails see Section 10.2) and thus have more realistic and better understood uncertainties. We note that many of the studies shown in the figure used overlapping data sets. For example, i) the Deason et al. and Grand et al. studies are based on the same stellar halo high velocity data, while the latter used an improved method calibrated with numerical simulations; ii) the Watkins et al., Posti \& Helmi, Vasiliev, and Eadie \& Jurić studies are based on the dynamics of halo globular clusters; and iii) the Callingham et al. and $\mathrm{Li}$ et al. studies used respectively the classical and all Galactic satellites and both methods are based on empirical phase-space distribution functions determined from the same hydrodynamical simulation. Nonetheless, the good agreement between the various estimates shown in Fig. 5 is indicating that we are converging towards a higher precision determination of the MW total mass.

\subsection{A summary of different methods}

We provide a summary of the various classes of methods in Table 1, including the tracers they have used and their modeling uncertainties.

Beyond the limitations listed in the table, all methods can be potentially affected by sample selection effects and hidden observational systematics. For example, both Vasiliev [383] and Posti et al. [316] in 2019 have assumed their sample of globular clusters to be complete, which might not be exactly true. In addition, since many of the previous measurements rely critically on the radial velocity of tracer objects from one or multiple spectroscopic surveys, systematic differences among different surveys may cause discrepancies between studies using different data or result in systematic biases in the same study combining different data. For example, comparisons of the radial velocities measured from LAMOST, RAVE, SEGUE and APOGEE have revealed a systematic offset of $\sim 5 \mathrm{~km} / \mathrm{s}$ between LAMOST and the other three surveys [e.g. 245, 340, 409]. The cause for the offset is still unknown, but it is shown to be independent of stellar properties and signal-to-noise ratios, and have been corrected for in the LAMOST data.

Besides, all methods can potentially suffer from uncertainties arising from a sparse number of tracers at large distances, and hence the measured total or virial mass of the MW largely relies on model extrapolations to large Galactocentric distances where there are not enough tracers to provide tight constraints. Furthermore, regardless of the method itself and the type of tracers used, parametrized potential models for both baryonic and dark matter have to be adopted for most of the measurements. Improper potential models can lead to illconstrained mass, especially for the extrapolated virial mass, which is largely model dependent. For studies trying to fit 
multiple components (disc, bulge and halo components for example), degeneracies exist among these different components. The readers can find such examples in Fig. 6 of Kafle et al. in 2014 [198]. A slightly overestimated stellar disk component brings a slightly underestimated enclosed dark matter mass, but a much more underestimated total mass, especially when no tracer objects in the outer halo are available.

In the next subsection, we summarize the role of modern numerical simulations. Since the mass distribution is known in simulations, testing the methods using cosmological simulations provides the most straight-forward way to validate model assumptions and to characterize systematic uncertainties. In addition, mock observations constructed from simulation data are also helpful for assessing sample selection effects.

\subsection{The role of numerical simulations}

Various methods rely on numerical simulations to infer the mass of our MW. Part of the studies used simulations to either directly infer plausible ranges for nuisance parameters or indirectly circumvent unconstrained parameters in their modeling. For example, as have been mentioned in Sec. 2, Smith et al. in 2007 [357], Piffl et al. in 2014 [312] and Deason et al. in 2019 [96] used cosmological simulations to infer the range of the power-law index in their high velocity tail distribution of halo stars. In addition, Xue et al. in 2008 [411] adopted the distributions of radial versus circular velocities of star particles in two simulated halos from hydrodynamical simulations. The Galactic circular velocities were determined by matching the observed distributions of radial versus circular velocities to those in simulations. Their approach helped to circumvent the problem of unknown proper motions or velocity anisotropies of tracers.

Some studies entirely depended on empirical relations [325] and probability distributions [9, 69, 72, 79, 156, 291] drawn from MW-like systems in simulations, which were then used to infer the virial mass of our MW. These efforts have been introduced in detail in Sec. 5.3 and Sec. 8 .

Other studies have relied on numerical simulations to validate their dynamical modeling, and some have calibrated their inferred mass by comparing between recovered and true masses in simulations. In the following, we summarize some of these attempts.

The early attempt of validating and calibrating the recovered MW mass through cosmological simulations can be traced back to the study of Li and White in 2008 [233], who have applied the timing argument approach (see details in Sec. 7) to halo pairs from the cosmological Millennium simulation. They calibrated the difference between MW timing mass and the true virial mass, using halo pairs similar to MW and Leo I. The usage of MW and Leo I pair requires the boundedness of Leo I to our MW, which was validated by Boylan-Kolchin et al. in 2013 [55] based on subhalos in cosmological simulations.

Peñarrubia et a. in 2016 [298] constrained the virial mass of our MW through the dynamics of local Hubble flow under the framework of the timing argument (see details in Sec. 7.2). Their measurement in 2016 has considered the effect of the LMC, and was calibrated by Peñarrubia and Fattahi in 2017 [297], by estimating the mass outside the virial radius of MW-like host halos using cosmological hydrodynamical simulations [298].

In terms of the distribution function method (see Sec. 5). Wang et al. in 2015 [392] have extended the approach to the NFW potentials, and have applied it to five MW-like galaxies from the Aquarius simulation, with stars generated from the particle tagging technique of [82]. The best-fit virial masses were biased from their true values to varying levels, and it was concluded that the cause for the bias varied from halo to halo. Besides, although it seems reasonable to assume that the binding energy and angular momentum terms can be decoupled from each other, the $\beta$ parameter in Eqn. 15 only stands for the true averaged velocity anisotropies for dark matter particles. For stars, their true velocity anisotropies are in fact larger than half of the best-fit power-law index in Eqn. 15. This reflects the inability of the adopted form of the distribution function to correctly match the actual distribution of stars. In fact, by applying the oPDF method (see Sec. 5.4 for details), which is a free-form distribution function method, to the Aquarius halos, it was shown that the major systematic biases found in [392] can be removed for all the halos. This demonstrates the biases found in [392] can be mostly attributed to the failure of the assumed function form in matching the actual distribution. Thus it is critical to avoid introducing incorrect or strong model assumptions in the construction of a distribution function.

Wang et al. in 2017 [390] have further applied the oPDF method to $\sim 1000 \mathrm{MW}$ sized dark matter halos from the cosmological Millennium II simulation and 24 MW/M31-like galaxies from the APOSTLE hydrodynamical simulations. On average, the best-fit halo properties were unbiased, while significant individual biases exist for most halos (see e.g., Fig. 6). Such individual biases can be as large as a factor of 2 to 3 when star particles were used as dynamical tracers. They found that these biases can be mostly attributed to correlated phase-space structures that violate the steady-state assumption. In the presence of phase correlations, the number of independent tracer particles is smaller than the actual number of tracers. This leads to stochastic biases in the parameter estimates, the distribution of which are determined 
Table 1 A summary of the classes of mass determination methods discussed in this review.

\begin{tabular}{|c|c|c|}
\hline Method & Tracer Population & Uncertainties and Difficulties \\
\hline \multirow[t]{2}{*}{$\begin{array}{l}\text { Escape velocity } \\
\text { (Sec. } 2)\end{array}$} & high velocity halo stars, & $\begin{array}{l}\text { Prior for the power law index, } k \text {, of the high velocity tail distribution. } \\
\text { Interlopers such as disk stars and substructures. }\end{array}$ \\
\hline & $\begin{array}{l}\text { exotic hypervelocity stars, } \\
\text { high velocity satellites }\end{array}$ & $\begin{array}{l}\text { Uncertain mechanisms of formation for hypervelocity stars. } \\
\text { Whether satellites are bound or not. }\end{array}$ \\
\hline $\begin{array}{l}\text { Rotation velocity } \\
\text { of the inner MW } \\
\text { (Sec. 3) }\end{array}$ & $\begin{array}{l}\text { terminal velocities of ISM within } R_{0} \text {, } \\
\text { circular velocities of masers/disk stars, } \\
\text { solar neighborhood stars }\end{array}$ & $\begin{array}{l}\text { Modeling the deviation from axis-symmetric assumption. } \\
\text { Need to combine with other methods and more distant tracers to } \\
\text { infer the mass out to large Galactocentric distances. }\end{array}$ \\
\hline $\begin{array}{l}\text { Spherical Jeans Equation } \\
\text { (Sec. 4) }\end{array}$ & halo stars, satellites, globular clusters & $\begin{array}{l}\text { Violation of the steady state and spherical assumptions. } \\
\text { Unknown velocity anisotropy due to unavailable proper motions. } \\
\text { Different tracer populations may have different velocity anisotropies. }\end{array}$ \\
\hline $\begin{array}{l}\text { Distribution function } \\
\text { (Sec. 5) }\end{array}$ & halo stars, satellites, globular clusters & $\begin{array}{l}\text { Violation of the steady state assumption due to phase correlations. } \\
\text { Violation of the spherical symmetry if spherical assumption is made. } \\
\text { Validity of the functional form. }\end{array}$ \\
\hline $\begin{array}{l}\text { modeling tidal debries } \\
\text { (Sec. 6) }\end{array}$ & stellar streams, survived progenitors & $\begin{array}{l}\text { Streams do not follow exactly the orbits of progenitors. } \\
\text { Contamination by stars not belonging to the stream. } \\
\text { Stripped stars might be re-accreted by the progenitor. } \\
\text { Single orbit fitting can introduce significant biases. } \\
\text { Tidal stripping and dynamical friction of the progenitor } \\
\text { is challenging to directly integrate backwards in time. } \\
\text { N-body simulations are expensive to explore the parameter space. }\end{array}$ \\
\hline $\begin{array}{l}\text { Timing argument } \\
\text { and LG dynamics } \\
\text { (Sec. 7) }\end{array}$ & $\begin{array}{l}\text { MW versus M31, } \\
\text { distant satellites of LG }\end{array}$ & $\begin{array}{l}\text { Non-zero tangential motions. } \\
\text { Mass contributed by massive satellites such as MCs. } \\
\text { Mass outside the virial radius contributing to the local reversal } \\
\text { of cosmic expansion. }\end{array}$ \\
\hline $\begin{array}{l}\text { Empirical distributions and } \\
\text { relations based on } \\
\text { simulated subhalos } \\
\text { (Sec. 8) }\end{array}$ & luminous satellite galaxies & $\begin{array}{l}\text { Simulation-based empirical relations and calibrations are largely } \\
\text { model dependent. } \\
\text { Simulated halos and subhalos do no fairly represent the properties } \\
\text { and merger histories of host and subhalos of our MW. }\end{array}$ \\
\hline
\end{tabular}

by the effective number of phase independent particles. As the oPDF method only makes use of the steady-state property (Equation (29)) in modeling the dynamics, these results suggest that there is only limited information that can be extracted from the data under a steady-state assumption. Such a limiting precision of steady-state modeling was further confirmed by Wang et al. (2018) [391], who found very similar amount of stochastic biases using the spherical Jeans equation (SJE, see section 4). Even though the methodologies of the oPDF and the Jeans equation are very different, they can be both derived from the collisionless Boltzmann equation under the steady-state assumption.

This steady-state information limit, though affecting all steady-state methods, is still different for different tracers. As found by Han et al. 2019 [173], the dynamical state of satellite galaxies is found to be close to that of dark matter particles. As a result, satellite galaxies exhibit a smaller stochastic bias compared to halo stars, as shown in Fig. 6.

In addition to picking a better steady-state tracer, another way of getting over the steady-state information limit is to use additional information beyond the steady-state assumption. Such information can be provided, for example, by the halo-dependent distribution of orbits that can be extracted semi-empirically from simulations. This is exactly what is done in the template-based distribution function method such as [236], [235] and [72]. As tested in [236] using simulated halos, their method is able to achieve a smaller systematic uncertainty than the oPDF method if a correct template is used.

Besides the dynamical state of the tracers, Wang et al. [390] have tested the validity of modeling the underlying potential as an NFW profile, and have found that biases arise when using tracers within $20 \mathrm{kpc}$ from the galaxy center, because the inner profiles deviate from the NFW model due to baryonic physics and the existence of galaxy disks. After excluding these innermost tracers, the NFW model returns on average unbiased mass estimates, and the scatter is very similar to that based on true potential templates directly extracted from the simulations. Deviations from spherical symmetry and the existence of a companion halo are also found to contribute to their systematic biases [390, 391]. For typical ap- 
plications of the SJE and derived mass estimators, additional systematic biases arise when $\beta$ is treated as a free but constant parameter [199, 391] and when the radial density profile of tracer objects is not properly measured or modeled [138].

In two very recent papers [121, 138], the Watkins et al. [393] mass estimator was validated and calibrated against simulations. Fritz et al. in 2020 [138] used dark matter only simulations to test and calibrate their measurements based on 45 satellite galaxies. After applying the mass estimator to simulations, significant biases were reported, which were mainly due to the deviation of satellite density profiles from a single power law. [138] also discussed systematic uncertainties arising from the LMC and LMC satellites. In another study, Erkal et al. in 2020 [121] pointed out that since the infall of the massive LMC can induce a substantial reflex motion in the MW [e.g. 120, 155, 302], this can make our MW, in particular the outer stellar halo, be out of equilibrium. Under this picture, [121] investigated how the non-equilibrium affects the performance of the Watkins et al. mass estimator and how this reflex motion affects the mass estimated by using Leo I [55]. They found if the mean reflex motion is not accounted for, the mass estimator can have systematic biases which are always positive and can be as large as $50 \%$. In addition, the LMC can significantly increase the speed of Leo I and cause overestimation of the MW mass.

A series of studies by Eadie et al. [111, 113, 114, 115] between 2015 and 2019 constrained the mass of our MW by modeling the phase-space distribution of globular clusters. In their paper published in 2018 [112], old and metal poor star particles in hydrodynamical simulations were used as globular cluster analogs to test and validate their hierarchical Bayesian-based dynamical method. Eadie et al. have found that the virial mass of the host halo in the simulations can sometimes be well recovered, but sometimes not. The main cause behind the incorrect estimates is due to the model itself: it has difficulties to simultaneously predict the inner and outer regions of the true mass profile. This limitation is probably due to the single power-law potential model [93]. Using only the outer-most tracers, where the underlying potential can be better approximated as a single power law, results can be improved to provide more accurate mass determinations.

Compared with satellite galaxies, halo stars and globular clusters, the modeling of spatially extended tidal streams is more complicated. A single orbit fitting can introduce significant biases, and integrations done backward in time makes it hard to directly incorporate tidal stripping and dynamical friction for the progenitor. Moreover, the tidal stream does not strictly follow the orbit of the progenitor. As we have mentioned in Sec. 6, N-body simulations are powerful approaches of modeling tidal streams and the rem- nant of their progenitors than single orbit fitting, but $\mathrm{N}$ body simulations are very expensive to explore the parameter space [105, 226, 227]. Other alternative and less expensive approaches such as particle releasing/spraying methods [34, 148, 222, 223] and semi-analytic approaches [104, 105] have been invoked as well.

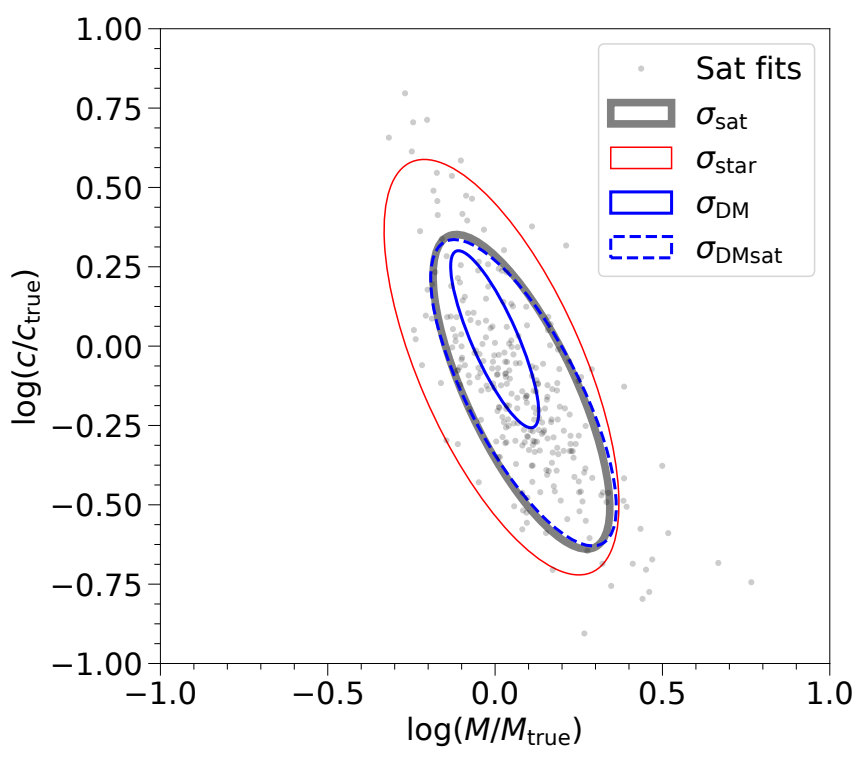

Figure 6 Biases of steady-state modeling using different tracers. A sample of MW-sized halos are selected from the Millennium-II simulation, with satellite galaxies extracted from the semi-analytic galaxy formation model [169]. The grey points show the fits to individual halos using satellites as tracers. The best-fit parameters are normalized by the true parameter values. The thick grey ellipse $\left(\sigma_{\text {sat }}\right)$ shows the total $1-\sigma$ scatter of the data points. The red $\left(\sigma_{\text {star }}\right)$ and blue $\left(\sigma_{\mathrm{DM}}\right)$ solid ellipses show the total scatters in the fits using stars and dark matter particles as tracers respectively [390]. For stars and dark matter particles, the number of tracers is $\sim 10^{5}$ for each halo, so that the total scatters are dominated by systematic biases. For satellites, the number of tracers is $\sim 100$. For a fair comparison between satellites and dark matter, the blue dashed ellipse $\left(\sigma_{\text {DMsat }}\right)$ shows the total scatter associated with dark matter particles down-sampled to the have the same number as that of satellites. Figure reproduced from [173].

Most of the earlier attempts of fitting the high velocity tail distribution of halo stars relied on numerical simulations to infer the plausible range of the nuisance parameter, and only until very recently, Grand et al. in 2019 [157] applied the method to simulated star particles in the suite of AURIGA simulations. They reported that substructures can affect the modeling of high velocity tail distributions. The median of recovered halo virial masses fell below the true values by $\sim 20 \%$, and the scatter can be as large as a factor of 2 .

The several examples just discussed highlight the essential role played by numerical simulations for validating and calibrating many mass estimation methods. However, these simulation-based validations and calibrations are not free of uncertainties. It is probably straight-forward and more robust 
to link satellite galaxies to simulated subhalos, which is less dependent of the baryonic physics adopted in the modeling. Dark matter particles are less dependent of baryonic physics as well, except for the very inner regions of dark matter halos, but it is not safe to directly extend validations based on dark matter particles to halo stars, as they have very different spatial and velocity distributions. Nowadays, star particles can be either directly simulated in hydrodynamical simulations or painted through semi-analytic approaches based on dark matter only simulations. Nonetheless, these simulations might still have limitations due to how the underlying baryonic physics is simulated or modeled, as our current understanding towards galaxy formation and evolution is still uncertain. Fortunately, our understanding of the infall and stripping of star particles under gravitational forces is more robust, and largely insensitive to baryonic physics. Compared with stars, there could be even more uncertainties behind the modeling of globular clusters, because the current understanding of the formation and evolution of globular clusters and their connections to dwarf galaxies is still uncertain [e.g. 425, 426]. Also, very importantly, as has been pointed out by [72] and [96], to test or design methods that take into account the specifics of the MW system, we would need a large sample of simulated systems whose mass growth is as close as possible to that of our own Galaxy. Despite these uncertain aspects, numerical simulations have been very helpful in aiding the dynamical modeling of various tracers and hence they are powerful tools for constraining the mass of our MW.

\subsection{Future prospects}

The near future is expected to bring a wealth of new observational data that will allow for a much more precise and hopefully accurate MW total mass determination. The abundance of new data will lead to new measurements of the MW mass distribution, such as the radial mass profile as well as the shape and orientation of the dark matter halo. To fully exploit the upcoming data, it is also necessary to improve the theoretical modeling of the MW system, such as going beyond spherical or axis-symmetric approximations, and accounting for non-equilibrium effects in the tracer distribution. In the following, we will discuss some of the upcoming Galactic observational campaigns as well as theoretical improvements that will be essential to correctly interpret and model the data.

The first and second data releases of the Gaia mission [140, 141, 301] have already revolutionized our understanding of the dynamics of the bulge, disk and halo stars, of the growth and chemical evolution, and of the orbits of stellar streams, globular clusters and satellite galaxies in our MW. And as we have demonstrated in Fig. 5, measurements of the
MW total mass using Gaia DR2 data are converging towards a higher precision. This has been possible due to the precise Gaia measurements of proper motions for billions of stars.

Future Gaia data releases ${ }^{11)}$ will improve further the data quality and the number of measured stars, which are essential for accurate modeling of the MW gravitational potential. However, the exquisite observational data set provided by the Gaia mission will mostly consist of nearby stars (e.g. most of the Gaia DR2 stars with useful proper motion and parallax measurements are found within $\sim 3 \mathrm{kpc}$ from the Sun). To accurately determine the MW mass, we need measurements at large Galactocentric radii, but at such large radii Gaia will only provide proper motion data for a small subset of intrinsically bright stars. This will be complemented by the deep and multi-epoch LSST imaging survey [192] that will provide proper motion measurements and photometric distances for individual stars $\sim 4$ magnitudes deeper than Gaia. This can potentially enable $6 \mathrm{D}$ phases space measurements for individual stars out to several tenths to hundreds of $\mathrm{kpc}$ and will be essential for determining the dynamics of the outer MW halo.

Among the billions of stars released by Gaia so far, only $\sim 7.2$ million stars have available radial velocities (these are mostly stars brighter than $G=13$ ). Encouragingly, many ground based follow-up spectroscopic surveys have been proposed to provide additional radial velocity measurements of faint stars, and especially of stars at large Galactocentric distances. To mention a few examples, these future surveys include the 4-metre Multi-Object Spectroscopic Telescope [4MOST; 89] in the south, the Subaru Prime Focus Spectroscopy [PFS; 370], LAMOST-2, The Milky Way Mapper (MWM) program of SDSS-V [214], the Dark Energy Spectroscopic Instrument [DESI; 102], the WEAVE survey [8], the MaunaKea Spectroscopic Explorer (MSE) in the north and the K2 RR Lyrae Survey along the Ecliptic [369].

Future surveys, such as LSST, are predicted to increase the number of satellite galaxies by a factor of two to ten, depending on model assumptions [282, 353]. Most such objects are predicted to be found at large distances and thus represent a potentially powerful window into the dynamics of the outer halo. Gravitationally bound objects, such as dwarf galaxies and globular clusters, are especially useful since one can average over the individual measurements of their member stars to obtain much more precise positions and velocities than possible for single stars. LSST will also help to identify more low-surface-brightness objects and stellar streams, which are largely missed by current photometric surveys.

The topic of this review, the total mass of the MW, is the first step in characterizing the mass distribution surrounding

11) For the most up-to-date schedule see https://www.cosmos.esa.int/web/gaia/release . 
our Galaxy. Future data sets will allow us to characterize the MW halo in much more details, such as in more precisely determining the concentration, shape and orientation of the dark matter halo. These aspects are also important for having an accurate determination of the MW total mass since, as the statistical uncertainties decrease, the errors will be dominated by systematic effects. For example, not accounting for the non-spherical shape of the dark matter halo can lead to biases in the inferred halo mass [e.g. 391]. Future models will also have to take into account that the shape and orientation of the halo changes with radius. In particular, previous works have argued that the outer MW halo is misaligned with the MW disk [227], which, given stability considerations, implies that the Galactic dark matter halo has a dramatic change in orientation from being aligned with the disk at distances $\lesssim 20 \mathrm{kpc}$ to being perpendicular to the stellar disk at large distances [349, 384].

The MW halo beyond several tens of kpc is likely to be out of equilibrium since the typical timescale at those distances is $\sim 1$ Gyr and longer. Furthermore, recent observational measurements as well as theoretical considerations suggest that the MW's brightest satellite, the LMC, is rather massive, having had a total mass at infall of about $\sim 2.5 \times 10^{11} \mathrm{M}_{\odot}$ $[78,120,122,137,298,348]$. This corresponds to $\sim 20 \%$ of the total MW mass, and thus the LMC is a major perturber of the Galactic potential. Previous studies have already highlighted the impact of a massive LMC on the orbit of the Sagittarius, Tucana III and Orphan streams [120, 124, 155], and have predicted significant LMC-induced disturbances in the density and velocity distribution of the MW stellar halo [144]. In particular, Erkal et al. in 2020 [121] pointed out that if the LMC is ignored, the MW mass can be overestimated by as much as $50 \%$. In addition, other massive satellite galaxies can also induce non-equilibrium features in the inner region of the MW, such as the Sagittarius perturbations of the stellar disk [e.g. 224]. To optimally use the wealth of upcoming observational data, future models will need to account for departures from equilibrium, which could be done by a combination of modeling individual perturbers, such as the LMC and Sagittarius dwarfs, and by statistically accounting for smaller non-equilibrium structures, such as diffuse stellar streams and shells, whose causes are harder to pinpoint.

New developments will also be required in terms of cosmological simulations that better describe the MW. The current state-of-the-art simulations of MW-mass systems [e.g. $158,337]$, while very useful, are rather limited since there are few (several tens) such systems, their resolution is insufficient to optimally compare with observations [e.g. 159], and the simulated systems do not necessarily reproduce the MW formation history. To better understand our Galaxy, future simulations should, beside having more realistic galaxy formation physics and higher resolution, be selected to reproduce key aspects in the evolution of the MW, such as a GaiaEnceladus-Sausage [14, 179] type of merger at high redshift followed by a long period of uneventful satellite galaxy accretions.

Acknowledgements: WW is grateful for useful suggestions made by Yipeng Jing, Carlos Frenk, Alis Deason and Zhao-yu Li. This work was supported by NSFC grant 11973032, 11890691, National Key Basic Research and Development Program of China (No.2018YFA0404504) and JSPS Grant-in-Aid for Scientific Research JP17K14271.

\section{References}

1 M. G. Abadi, J. F. Navarro, and M. Steinmetz. Stars beyond galaxies: the origin of extended luminous haloes around galaxies. MNRAS, 365:747-758, Jan. 2006.

2 I. Ablimit and G. Zhao. The Milky Way's Circular Velocity Curve and Its Constraint on the Galactic Mass with RR Lyrae Stars. ApJ, 846(1):10, Sep 2017.

3 J. An, N. W. Evans, and A. J. Deason. Mass estimators in the Gaia era. MNRAS, 420:2562-2568, Mar. 2012.

4 J. H. An and N. W. Evans. Modified virial formulae and the theory of mass estimators. MNRAS, 413:17441752, May 2011.

5 J. N. Bahcall and S. Tremaine. Methods for determining the masses of spherical systems. I - Test particles around a point mass. ApJ, 244:805-819, Mar. 1981.

6 A. T. Bajkova and V. V. Bobylev. Rotation curve and mass distribution in the Galaxy from the velocities of objects at distances up to $200 \mathrm{kpc}$. Astronomy Letters, 42(9):567-582, Sep 2016.

7 E. Balbinot, B. X. Santiago, L. N. da Costa, M. Makler, and M. A. G. Maia. The tidal tails of NGC 2298. MNRAS, 416:393-402, Sept. 2011.

8 M. Balcells. The WEAVE spectrograph on the WHT: plans for radial velocity and chemistry surveys of the northern and equatorial Milky Way. In EAS Publications Series, volume 67-68, pages 227-230, Jul 2014.

9 C. Barber, E. Starkenburg, J. F. Navarro, A. W. McConnachie, and A. Fattahi. The orbital ellipticity of satellite galaxies and the mass of the Milky Way. MNRAS, 437:959-967, Jan. 2014. 
10 G. Battaglia, A. Helmi, H. Morrison, P. Harding, E. W. Olszewski, M. Mateo, K. C. Freeman, J. Norris, and S. A. Shectman. The radial velocity dispersion profile of the Galactic halo: constraining the density profile of the dark halo of the Milky Way. MNRAS, 364:433442, Dec. 2005.

11 H. Baumgardt, M. Hilker, A. Sollima, and A. Bellini. Mean proper motions, space orbits, and velocity dispersion profiles of Galactic globular clusters derived from Gaia DR2 data. MNRAS, 482:5138-5155, Feb. 2019.

12 E. F. Bell, D. B. Zucker, V. Belokurov, S. Sharma, K. V. Johnston, J. S. Bullock, D. W. Hogg, K. Jahnke, and et al. The Accretion Origin of the Milky Way's Stellar Halo. ApJ, 680:295-311, June 2008.

13 A. M. Beloborodov and Y. Levin. Orbital Roulette: A New Method of Gravity Estimation from Observed Motions. ApJ, 613:224-237, Sept. 2004.

14 V. Belokurov, D. Erkal, N. W. Evans, S. E. Koposov, and A. J. Deason. Co-formation of the disc and the stellar halo. MNRAS, 478(1):611-619, Jul 2018.

15 V. Belokurov, N. W. Evans, M. J. Irwin, P. C. Hewett, and M. I. Wilkinson. The Discovery of Tidal Tails around the Globular Cluster NGC 5466. ApJ, 637:L29L32, Jan. 2006.

16 V. Belokurov, N. W. Evans, M. J. Irwin, D. LyndenBell, B. Yanny, S. Vidrih, G. Gilmore, G. Seabroke, and et al. An Orphan in the "Field of Streams". ApJ, 658:337-344, Mar. 2007.

17 V. Belokurov, D. B. Zucker, N. W. Evans, G. Gilmore, S. Vidrih, D. M. Bramich, H. J. Newberg, R. F. G. Wyse, and et al. The Field of Streams: Sagittarius and Its Siblings. ApJ, 642:L137-L140, May 2006.

18 D. Benisty, E. I. Guendelman, and O. Lahav. Milky Way and Andromeda past-encounters in different gravity models: the impact on the estimated Local Group mass. arXiv e-prints, page arXiv:1904.03153, Apr 2019.

19 A. J. Benson, C. G. Lacey, C. M. Baugh, S. Cole, and C. S. Frenk. The effects of photoionization on galaxy formation - I. Model and results at $\mathrm{z}=0$. MNRAS, 333(1):156-176, Jun 2002.

20 E. J. Bernard, A. M. N. Ferguson, E. F. Schlafly, M. Abbas, E. F. Bell, N. R. Deacon, N. F. Martin, H.-W.
Rix, and et al. Serendipitous discovery of a thin stellar stream near the Galactic bulge in the Pan-STARRS1 3 $\pi$ Survey. MNRAS, 443:L84-L88, Sept. 2014.

21 G. Besla, N. Kallivayalil, L. Hernquist, B. Robertson, T. J. Cox, R. P. van der Marel, and C. Alcock. Are the Magellanic Clouds on Their First Passage about the Milky Way? ApJ, 668:949-967, Oct. 2007.

22 G. Besla, N. Kallivayalil, L. Hernquist, R. P. van der Marel, T. J. Cox, and D. Kereš. Simulations of the Magellanic Stream in a First Infall Scenario. ApJ, 721:L97L101, Oct. 2010.

23 P. Bhattacharjee, S. Chaudhury, and S. Kundu. Rotation Curve of the Milky Way out to $200 \mathrm{kpc}$. ApJ, 785:63, Apr. 2014.

24 J. Binney. Distribution functions for the Milky Way. MNRAS, 401:2318-2330, Feb. 2010.

25 J. Binney. Actions for axisymmetric potentials. MNRAS, 426:1324-1327, Oct. 2012.

26 J. Binney and P. McMillan. Models of our Galaxy - II. MNRAS, 413:1889-1898, May 2011.

27 J. Binney and P. J. McMillan. Torus mapper: a code for dynamical models of galaxies. MNRAS, 456:19821998, Feb. 2016.

28 J. Binney and S. Tremaine. Galactic Dynamics: Second Edition. Princeton University Press, 2008.

29 J. Binney and L. K. Wong. Modelling the Milky Way's globular cluster system. MNRAS, 467:2446-2457, May 2017.

30 S. A. Bird, X.-X. Xue, C. Liu, J. Shen, C. Flynn, and C. Yang. Anisotropy of the Milky Way's Stellar Halo Using K Giants from LAMOST and Gaia. AJ, 157(3):104, Mar 2019.

31 J. Bland-Hawthorn and O. Gerhard. The Galaxy in Context: Structural, Kinematic, and Integrated Properties. ARA\&A, 54:529-596, Sep 2016.

32 V. V. Bobylev, A. T. Bajkova, and A. O. Gromov. Refinement of the parameters of three selected model Galactic potentials based on the velocities of objects at distances up to $200 \mathrm{kpc}$. Astronomy Letters, 43(4):241251, Apr 2017.

33 A. Bonaca, M. Geha, and N. Kallivayalil. A Cold Milky Way Stellar Stream in the Direction of Triangulum. ApJ, 760:L6, Nov. 2012. 
34 A. Bonaca, M. Geha, A. H. W. Küpper, J. Diemand, K. V. Johnston, and D. W. Hogg. Milky Way Mass and Potential Recovery Using Tidal Streams in a Realistic Halo. ApJ, 795:94, Nov. 2014.

35 A. Bonaca and D. W. Hogg. The information content in cold stellar streams. ArXiv e-prints, Apr. 2018.

36 A. Bonaca and D. W. Hogg. The Information Content in Cold Stellar Streams. ApJ, 867(2):101, Nov 2018.

37 N. A. Bond, Ž. Ivezić, B. Sesar, M. Jurić, J. A. Munn, A. Kowalski, S. Loebman, R. Roškar, and et al. The Milky Way Tomography with SDSS. III. Stellar Kinematics. ApJ, 716:1-29, June 2010.

38 D. Boubert, D. Erkal, N. W. Evans, and R. G. Izzard. Hypervelocity runaways from the Large Magellanic Cloud. MNRAS, 469:2151-2162, Aug. 2017.

39 D. Boubert and N. W. Evans. A Dipole on the Sky: Predictions for Hypervelocity Stars from the Large Magellanic Cloud. ApJ, 825:L6, July 2016.

40 D. Boubert, J. Guillochon, K. Hawkins, I. Ginsburg, N. W. Evans, and J. Strader. Revisiting hypervelocity stars after Gaia DR2. MNRAS, 479:2789-2795, Sept. 2018.

41 J. Bovy. Dynamical Modeling of Tidal Streams. ApJ, 795:95, Nov. 2014.

42 J. Bovy. galpy: A python Library for Galactic Dynamics. ApJS, 216:29, Feb. 2015.

43 J. Bovy. Detecting the Disruption of Dark-Matter Halos with Stellar Streams. Physical Review Letters, 116(12):121301, Mar. 2016.

44 J. Bovy, C. Allende Prieto, T. C. Beers, D. Bizyaev, L. N. da Costa, K. Cunha, G. L. Ebelke, D. J. Eisenstein, and et al. The Milky Way's Circular-velocity Curve between 4 and $14 \mathrm{kpc}$ from APOGEE data. ApJ, 759:131, Nov. 2012.

45 J. Bovy, A. Bahmanyar, T. K. Fritz, and N. Kallivayalil. The Shape of the Inner Milky Way Halo from Observations of the Pal 5 and GD-1 Stellar Streams. ApJ, 833:31, Dec. 2016.

46 J. Bovy, D. Erkal, and J. L. Sanders. Linear perturbation theory for tidal streams and the small-scale CDM power spectrum. MNRAS, 466:628-668, Apr. 2017.

47 J. Bovy, D. W. Hogg, and H.-W. Rix. Galactic Masers and the Milky Way Circular Velocity. ApJ, 704:17041709, Oct. 2009.
48 J. Bovy, I. Murray, and D. W. Hogg. Dynamical Inference from a Kinematic Snapshot: The Force Law in the Solar System. ApJ, 711:1157-1167, Mar. 2010.

49 J. Bovy and H.-W. Rix. A Direct Dynamical Measurement of the Milky Way's Disk Surface Density Profile, Disk Scale Length, and Dark Matter Profile at $4 \mathrm{kpc}$. ApJ, 779:115, Dec. 2013.

50 J. Bovy, H.-W. Rix, and D. W. Hogg. The Milky Way Has No Distinct Thick Disk. ApJ, 751:131, June 2012.

51 J. Bovy and S. Tremaine. On the Local Dark Matter Density. ApJ, 756:89, Sept. 2012.

52 A. Bowden, V. Belokurov, and N. W. Evans. Dipping our toes in the water: first models of GD-1 as a stream. MNRAS, 449:1391-1400, May 2015.

53 M. Boylan-Kolchin, G. Besla, and L. Hernquist. Dynamics of the Magellanic Clouds in a Lambda cold dark matter universe. MNRAS, 414:1560-1572, June 2011.

54 M. Boylan-Kolchin, J. S. Bullock, and M. Kaplinghat. Too big to fail? The puzzling darkness of massive Milky Way subhaloes. MNRAS, 415:L40-L44, July 2011.

55 M. Boylan-Kolchin, J. S. Bullock, S. T. Sohn, G. Besla, and R. P. van der Marel. The Space Motion of Leo I: The Mass of the Milky Way's Dark Matter Halo. ApJ, 768:140, May 2013.

56 M. Boylan-Kolchin, V. Springel, S. D. M. White, and A. Jenkins. There's no place like home? Statistics of Milky Way-mass dark matter haloes. MNRAS, 406:896-912, Aug. 2010.

57 M. Boylan-Kolchin, V. Springel, S. D. M. White, A. Jenkins, and G. Lemson. Resolving cosmic structure formation with the Millennium-II Simulation. MNRAS, 398:1150-1164, Sept. 2009.

58 B. C. Bromley, S. J. Kenyon, W. R. Brown, and M. J. Geller. Runaway Stars, Hypervelocity Stars, and Radial Velocity Surveys. ApJ, 706:925-940, Dec. 2009.

59 B. C. Bromley, S. J. Kenyon, M. J. Geller, E. Barcikowski, W. R. Brown, and M. J. Kurtz. Hypervelocity Stars: Predicting the Spectrum of Ejection Velocities. ApJ, 653:1194-1202, Dec. 2006.

60 W. R. Brown. Hypervelocity Stars. ARA\&A, 53:15-49, Aug. 2015. 
61 W. R. Brown, M. J. Geller, and S. J. Kenyon. MMT Hypervelocity Star Survey. ApJ, 690:1639-1647, Jan. 2009.

62 W. R. Brown, M. J. Geller, and S. J. Kenyon. MMT Hypervelocity Star Survey. III. The Complete Survey. ApJ, 787:89, May 2014.

63 W. R. Brown, M. J. Geller, S. J. Kenyon, M. J. Kurtz, and B. C. Bromley. Hypervelocity Stars. II. The Bound Population. ApJ, 660:311-318, May 2007.

64 W. R. Brown, M. J. Geller, S. J. Kenyon, M. J. Kurtz, and B. C. Bromley. Hypervelocity Stars. III. The Space Density and Ejection History of Main-Sequence Stars from the Galactic Center. ApJ, 671:1708-1716, Dec. 2007.

65 W. R. Brown, M. G. Lattanzi, S. J. Kenyon, and M. J. Geller. Gaia and the Galactic Center Origin of Hypervelocity Stars. ApJ, 866:39, Oct. 2018.

66 A. Brunthaler, M. J. Reid, K. M. Menten, X.-W. Zheng, A. Bartkiewicz, Y. K. Choi, T. Dame, K. Hachisuka, and et al. The Bar and Spiral Structure Legacy (BeSSeL) survey: Mapping the Milky Way with VLBI astrometry. Astronomische Nachrichten, 332:461, June 2011.

67 J. S. Bullock and K. V. Johnston. Tracing Galaxy Formation with Stellar Halos. I. Methods. ApJ, 635:931949, Dec. 2005.

68 J. S. Bullock, A. V. Kravtsov, and D. H. Weinberg. Reionization and the Abundance of Galactic Satellites. ApJ, 539(2):517-521, Aug 2000.

69 M. T. Busha, P. J. Marshall, R. H. Wechsler, A. Klypin, and J. Primack. The Mass Distribution and Assembly of the Milky Way from the Properties of the Magellanic Clouds. ApJ, 743:40, Dec. 2011.

70 M. T. Busha, R. H. Wechsler, P. S. Behroozi, B. F. Gerke, A. A. Klypin, and J. R. Primack. Statistics of Satellite Galaxies around Milky-Way-like Hosts. ApJ, 743:117, Dec. 2011.

71 T. M. Callingham, M. Cautun, A. J. Deason, C. S. Frenk, R. J. J. Grand, F. Marinacci, and R. Pakmor. The orbital phase space of contracted dark matter halos. arXiv e-prints, page arXiv:2001.07742, Jan 2020.

72 T. M. Callingham, M. Cautun, A. J. Deason, C. S. Frenk, W. Wang, F. A. Gómez, R. J. J. Grand, F. Marinacci, and R. Pakmor. The mass of the Milky Way from satellite dynamics. MNRAS, 484:5453-5467, Apr. 2019.

73 R. Capuzzo-Dolcetta and G. Fragione. High-velocity stars from close interaction of a globular cluster and a supermassive black hole. MNRAS, 454:2677-2690, Dec. 2015.

74 R. G. Carlberg. Star Stream Folding by Dark Galactic Subhalos. ApJ, 705:L223-L226, Nov. 2009.

75 J. L. Carlin, W. Yam, D. I. Casetti-Dinescu, B. A. Willett, H. J. Newberg, S. R. Majewski, and T. M. Girard. The Origin of the Virgo Stellar Substructure. ApJ, 753:145, July 2012.

76 M. Cautun, A. Benitez-Llambay, A. J. Deason, C. S. Frenk, A. Fattahi, F. A. Gómez, R. J. J. Grand, K. A. Oman, J. F. Navarro, and C. M. Simpson. The Milky Way total mass profile as inferred from Gaia DR2. arXiv e-prints, page arXiv:1911.04557, Nov 2019.

77 M. Cautun, S. Bose, C. S. Frenk, Q. Guo, J. Han, W. A. Hellwing, T. Sawala, and W. Wang. Planes of satellite galaxies: when exceptions are the rule. MNRAS, 452(4):3838-3852, Oct 2015.

78 M. Cautun, A. J. Deason, C. S. Frenk, and S. McAlpine. The aftermath of the Great Collision between our Galaxy and the Large Magellanic Cloud. MNRAS, 483(2):2185-2196, Feb 2019.

79 M. Cautun, C. S. Frenk, R. van de Weygaert, W. A. Hellwing, and B. J. T. Jones. Milky Way mass constraints from the Galactic satellite gap. MNRAS, 445:2049-2060, Dec. 2014.

80 M. Cautun, W. A. Hellwing, R. van de Weygaert, C. S. Frenk, B. J. T. Jones, and T. Sawala. Subhalo statistics of galactic haloes: beyond the resolution limit. MNRAS, 445(2):1820-1835, Dec 2014.

81 M. Cautun, W. Wang, C. S. Frenk, and T. Sawala. A new spin on discs of satellite galaxies. MNRAS, 449(3):2576-2587, May 2015.

82 A. P. Cooper, S. Cole, C. S. Frenk, S. D. M. White, J. Helly, A. J. Benson, G. De Lucia, A. Helmi, and et al. Galactic stellar haloes in the CDM model. MNRAS, 406:744-766, Aug. 2010.

83 R. A. Crain, J. Schaye, R. G. Bower, M. Furlong, M. Schaller, T. Theuns, C. Dalla Vecchia, C. S. Frenk, and et al. The EAGLE simulations of galaxy formation: calibration of subgrid physics and model variations. MNRAS, 450:1937-1961, June 2015. 
84 M. Creze, E. Chereul, O. Bienayme, and C. Pichon. The distribution of nearby stars in phase space mapped by Hipparcos. I. The potential well and local dynamical mass. A\&A, 329:920-936, Jan. 1998.

85 P. Cuddeford. An analytic inversion for anisotropic spherical galaxies. MNRAS, 253:414-426, Dec. 1991.

86 X.-Q. Cui, Y.-H. Zhao, Y.-Q. Chu, G.-P. Li, Q. Li, L.P. Zhang, H.-J. Su, Z.-Q. Yao, and et al. The Large Sky Area Multi-Object Fiber Spectroscopic Telescope (LAMOST). Research in Astronomy and Astrophysics, 12:1197-1242, Sept. 2012.

87 P. Das and J. Binney. Characterizing stellar halo populations - I. An extended distribution function for halo $\mathrm{K}$ giants. MNRAS, 460:1725-1738, Aug. 2016.

88 P. Das, A. Williams, and J. Binney. Characterizing stellar halo populations II: the age gradient in blue horizontal-branch stars. MNRAS, 463(3):3169-3185, Dec 2016.

89 R. S. de Jong, S. C. Barden, O. Bellido-Tirado, J. G. Brynnel, S. Frey, D. Giannone, R. Haynes, D. Johl, and et al. 4MOST: the 4-metre Multi-Object Spectroscopic Telescope project at preliminary design review. In Ground-based and Airborne Instrumentation for Astronomy VI, volume 9908 of Proc. SPIE, page 99081O, Aug. 2016.

90 T. de Zeeuw. Elliptical galaxies with separable potentials. MNRAS, 216:273-334, Sept. 1985.

91 A. J. Deason, V. Belokurov, and N. W. Evans. Rotation of halo populations in the Milky Way and M31. MNRAS, 411:1480-1494, Mar. 2011.

92 A. J. Deason, V. Belokurov, and N. W. Evans. The Milky Way stellar halo out to $40 \mathrm{kpc}$ : squashed, broken but smooth. MNRAS, 416:2903-2915, Oct. 2011.

93 A. J. Deason, V. Belokurov, N. W. Evans, and J. An. Broken degeneracies: the rotation curve and velocity anisotropy of the Milky Way halo. MNRAS, 424:L44L48, July 2012.

94 A. J. Deason, V. Belokurov, N. W. Evans, and K. V. Johnston. Broken and Unbroken: The Milky Way and M31 Stellar Halos. ApJ, 763:113, Feb. 2013.

95 A. J. Deason, V. Belokurov, N. W. Evans, S. E. Koposov, R. J. Cooke, J. Peñarrubia, C. F. P. Laporte, M. Fellhauer, and et al. The cold veil of the Milky Way stellar halo. MNRAS, 425:2840-2853, Oct. 2012.
96 A. J. Deason, A. Fattahi, V. Belokurov, N. W. Evans, R. J. J. Grand, F. Marinacci, and R. Pakmor. The local high-velocity tail and the Galactic escape speed. MNRAS, 485:3514-3526, May 2019.

97 A. J. Deason, R. P. Van der Marel, P. Guhathakurta, S. T. Sohn, and T. M. Brown. The Velocity Anisotropy of Distant Milky Way Halo Stars from Hubble Space Telescope Proper Motions. ApJ, 766:24, Mar. 2013.

98 N. Deg and L. Widrow. Incorporating streams into Milky Way models. MNRAS, 439:2678-2686, Apr. 2014.

99 W. Dehnen and J. Binney. Mass models of the Milky Way. MNRAS, 294:429, Mar. 1998.

100 W. Dehnen, D. E. McLaughlin, and J. Sachania. The velocity dispersion and mass profile of the Milky Way. MNRAS, 369:1688-1692, July 2006.

101 L.-C. Deng, H. J. Newberg, C. Liu, J. L. Carlin, T. C. Beers, L. Chen, Y.-Q. Chen, N. Christlieb, and et al. LAMOST Experiment for Galactic Understanding and Exploration (LEGUE) - The survey's science plan. Research in Astronomy and Astrophysics, 12:735-754, July 2012.

102 DESI Collaboration, A. Aghamousa, J. Aguilar, S. Ahlen, S. Alam, L. E. Allen, C. Allende Prieto, J. Annis, and et al. The DESI Experiment Part I: Science,Targeting, and Survey Design. arXiv e-prints, page arXiv:1611.00036, Oct 2016.

103 J. D. Diaz, S. E. Koposov, M. Irwin, V. Be2014MNRAS.443.1688Dlokurov, and N. W. Evans. Balancing mass and momentum in the Local Group. MNRAS, 443:1688-1703, Sept. 2014.

104 M. I. P. Dierickx and A. Loeb. An Upper Limit on the Milky Way Mass from the Orbit of the Sagittarius Dwarf Satellite. ApJ, 847:42, Sept. 2017.

105 M. I. P. Dierickx and A. Loeb. Predicted Extension of the Sagittarius Stream to the Milky Way Virial Radius. ApJ, 836:92, Feb. 2017.

106 D. I. Dinescu, T. M. Girard, W. F. van Altena, and C. E. López. Absolute Proper Motion of the Sagittarius Dwarf Galaxy and of the Outer Regions of the Milky Way Bulge. ApJ, 618:L25-L28, Jan. 2005.

107 D. I. Dinescu, B. A. Keeney, S. R. Majewski, and T. M. Girard. Absolute Proper Motion of the Fornax 
Dwarf Spheroidal Galaxy from Photographic and Hubble Space Telescope WFPC2 Data. AJ, 128:687-699, Aug. 2004.

108 D. I. Dinescu, D. Martínez-Delgado, T. M. Girard, J. Peñarrubia, H.-W. Rix, D. Butler, and W. F. van Altena. Absolute Proper Motion of the Canis Major Dwarf Galaxy Candidate. ApJ, 631:L49-L52, Sept. 2005.

109 E. D’Onghia and G. Lake. Small Dwarf Galaxies within Larger Dwarfs: Why Some Are Luminous while Most Go Dark. ApJ, 686:L61, Oct. 2008.

110 A. R. Duffy, J. Schaye, S. T. Kay, and C. Dalla Vecchia. Dark matter halo concentrations in the Wilkinson Microwave Anisotropy Probe year 5 cosmology. MNRAS, 390:L64-L68, Oct. 2008.

111 G. Eadie and M. Jurić. The Cumulative Mass Profile of the Milky Way as Determined by Globular Cluster Kinematics from Gaia DR2. ApJ, 875(2):159, Apr 2019.

112 G. Eadie, B. Keller, and W. E. Harris. Estimating the Milky Way's Mass via Hierarchical Bayes: A Blind Test on MUGS2 Simulated Galaxies. ApJ, 865:72, Sept. 2018.

113 G. M. Eadie and W. E. Harris. Bayesian Mass Estimates of the Milky Way: The Dark and Light Sides of Parameter Assumptions. ApJ, 829:108, Oct. 2016.

114 G. M. Eadie, W. E. Harris, and L. M. Widrow. Estimating the Galactic Mass Profile in the Presence of Incomplete Data. ApJ, 806:54, June 2015.

115 G. M. Eadie, A. Springford, and W. E. Harris. Bayesian Mass Estimates of the Milky Way: Including Measurement Uncertainties with Hierarchical Bayes. ApJ, 835:167, Feb. 2017.

116 A. S. Eddington. The distribution of stars in globular clusters. MNRAS, 76:572-585, May 1916.

117 A.-C. Eilers, D. W. Hogg, H.-W. Rix, and M. Ness. The Circular Velocity Curve of the Milky Way from 5 to 25 kpc. arXiv e-prints, Oct. 2018.

118 D. J. Eisenstein, D. H. Weinberg, E. Agol, H. Aihara, C. Allende Prieto, S. F. Anderson, J. A. Arns, É. Aubourg, and et al. SDSS-III: Massive Spectroscopic Surveys of the Distant Universe, the Milky Way, and Extra-Solar Planetary Systems. AJ, 142:72, Sept. 2011.
119 D. Erkal, V. Belokurov, J. Bovy, and J. L. Sanders. The number and size of subhalo-induced gaps in stellar streams. MNRAS, 463:102-119, Nov. 2016.

120 D. Erkal, V. Belokurov, C. F. P. Laporte, S. E. Koposov, T. S. Li, C. J. Grillmair, N. Kallivayalil, A. M. PriceWhelan, N. W. Evans, K. Hawkins, D. Hendel, C. Mateu, J. F. Navarro, A. del Pino, C. T. Slater, S. T. Sohn, and Orphan Aspen Treasury Collaboration. The total mass of the Large Magellanic Cloud from its perturbation on the Orphan stream. MNRAS, 487(2):26852700, Aug 2019.

121 D. Erkal, V. Belokurov, and D. L. Parkin. Equilibrium models of the Milky Way mass are biased high by the LMC. arXiv e-prints, page arXiv:2001.11030, Jan 2020.

122 D. Erkal and V. A. Belokurov. Limit on the LMC mass from a census of its satellites. arXiv e-prints, page arXiv:1907.09484, Jul 2019.

123 D. Erkal, D. Boubert, A. Gualandris, N. W. Evans, and F. Antonini. A hypervelocity star with a Magellanic origin. MNRAS, 483:2007-2013, Feb. 2019.

124 D. Erkal, T. S. Li, S. E. Koposov, V. Belokurov, E. Balbinot, K. Bechtol, B. Buncher, A. Drlica-Wagner, and et al. Modelling the Tucana III stream - a close passage with the LMC. MNRAS, 481(3):3148-3159, Dec 2018.

125 N. W. Evans, J. An, and A. J. Deason. Accurate Mass Estimators for Navarro-Frenk-White Halos. ApJ, 730:L26, Apr. 2011.

126 N. W. Evans, P. T. de Zeeuw, and D. Lynden-Bell. The flattened isochrone. MNRAS, 244:111-129, May 1990.

127 N. W. Evans, R. M. Hafner, and P. T. de Zeeuw. Simple three-integral scale-free galaxy models. MNRAS, 286:315-328, Apr. 1997.

128 A. Eyre and J. Binney. Fitting orbits to tidal streams with proper motions. MNRAS, 399:L160-L163, Oct. 2009.

129 A. Eyre and J. Binney. Locating the orbits delineated by tidal streams. MNRAS, 400:548-560, Nov. 2009.

130 A. Fattahi, J. F. Navarro, and C. S. Frenk. The missing dwarf galaxies of the Local Group. arXiv e-prints, page arXiv:1907.02463, Jul 2019.

131 A. Fattahi, J. F. Navarro, T. Sawala, C. S. Frenk, K. A. Oman, R. A. Crain, M. Furlong, M. Schaller, and et al. 
The APOSTLE project: Local Group kinematic mass constraints and simulation candidate selection. $\mathrm{MN}$ RAS, 457:844-856, Mar. 2016.

132 M. Fellhauer, V. Belokurov, N. W. Evans, M. I. Wilkinson, D. B. Zucker, G. Gilmore, M. J. Irwin, D. M. Bramich, and et al. The Origin of the Bifurcation in the Sagittarius Stream. ApJ, 651:167-173, Nov. 2006.

133 G. Fragione and R. Capuzzo-Dolcetta. High-velocity stars from the interaction of a globular cluster and a massive black hole binary. MNRAS, 458:2596-2603, May 2016.

134 G. Fragione and A. Loeb. Constraining the Milky Way mass with hypervelocity stars. New A, 55:32-38, Aug. 2017.

135 P. M. Frinchaboy and S. R. Majewski. Open Clusters as Galactic Disk Tracers. I. Project Motivation, Cluster Membership, and Bulk Three-Dimensional Kinematics. AJ, 136:118-145, July 2008.

136 T. K. Fritz, G. Battaglia, M. S. Pawlowski, N. Kallivayalil, R. van der Marel, S. T. Sohn, C. Brook, and G. Besla. Gaia DR2 proper motions of dwarf galaxies within $420 \mathrm{kpc}$. Orbits, Milky Way mass, tidal influences, planar alignments, and group infall. A\&A, 619:A103, Nov. 2018.

137 T. K. Fritz, R. Carrera, G. Battaglia, and S. Taibi. Gaia DR 2 and VLT/FLAMES search for new satellites of the LMC. A\&A, 623:A129, Mar 2019.

138 T. K. Fritz, A. Di Cintio, G. Battaglia, C. Brook, and S. Taibi. The mass of our Galaxy from satellite proper motions in the Gaia era. arXiv e-prints, page arXiv:2001.02651, Jan 2020.

139 T. Fukushima, M. Chiba, M. Tanaka, K. Hayashi, D. Homma, S. Okamoto, Y. Komiyama, M. Tanaka, and et al. The stellar halo of the Milky Way traced by blue horizontal-branch stars in the Subaru Hyper SuprimeCam Survey. PASJ, page 68, Jun 2019.

140 Gaia Collaboration, A. G. A. Brown, A. Vallenari, T. Prusti, J. H. J. de Bruijne, C. Babusiaux, C. A. L. Bailer-Jones, M. Biermann, D. W. Evans, L. Eyer, F. Jansen, C. Jordi, and et al. Gaia Data Release 2. Summary of the contents and survey properties. A\&A, 616:A1, Aug 2018.

141 Gaia Collaboration, A. G. A. Brown, A. Vallenari, T. Prusti, J. H. J. de Bruijne, F. Mignard, and et al. Gaia
Data Release 1. Summary of the astrometric, photometric, and survey properties. A\&A, 595:A2, Nov 2016.

142 Gaia Collaboration, A. Helmi, F. van Leeuwen, P. J. McMillan, D. Massari, T. Antoja, A. C. Robin, L. Lindegren, and et al. Gaia Data Release 2. Kinematics of globular clusters and dwarf galaxies around the Milky Way. A\&A, 616:A12, Aug. 2018.

143 C. Gallart, E. J. Bernard, C. B. Brook, T. Ruiz-Lara, S. Cassisi, V. Hill, and M. Monelli. Uncovering the birth of the Milky Way through accurate stellar ages with Gaia. Nature Astronomy, 3:932-939, Jul 2019.

144 N. Garavito-Camargo, G. Besla, C. F. P. Laporte, K. V. Johnston, F. A. Gómez, and L. L. Watkins. Hunting for the Dark Matter Wake Induced by the Large Magellanic Cloud. ApJ, 884(1):51, Oct 2019.

145 S. Garbari, C. Liu, J. I. Read, and G. Lake. A new determination of the local dark matter density from the kinematics of K dwarfs. MNRAS, 425:1445-1458, Sept. 2012.

146 L. T. Gardiner, T. Sawa, and M. Fujimoto. Numerical Simulations of the Magellanic System - Part One - Orbits of the Magellanic Clouds and the Global Gas Distribution. MNRAS, 266:567, Feb. 1994.

147 S. Genel, M. Vogelsberger, V. Springel, D. Sijacki, D. Nelson, G. Snyder, V. Rodriguez-Gomez, P. Torrey, and L. Hernquist. Introducing the Illustris project: the evolution of galaxy populations across cosmic time. MNRAS, 445:175-200, Nov. 2014.

148 S. L. J. Gibbons, V. Belokurov, and N. W. Evans. 'Skinny Milky Way please', says Sagittarius. MNRAS, 445:3788-3802, Dec. 2014.

149 S. Gillessen, F. Eisenhauer, S. Trippe, T. Alexander, R. Genzel, F. Martins, and T. Ott. Monitoring Stellar Orbits Around the Massive Black Hole in the Galactic Center. ApJ, 692:1075-1109, Feb. 2009.

150 G. Gilmore, S. Randich, M. Asplund, J. Binney, P. Bonifacio, J. Drew, S. Feltzing, A. Ferguson, and et al. The Gaia-ESO Public Spectroscopic Survey. The Messenger, 147:25-31, Mar. 2012.

151 I. Ginsburg and A. Loeb. The fate of former companions to hypervelocity stars originating at the Galactic Centre. MNRAS, 368:221-225, May 2006.

152 I. Ginsburg and A. Loeb. Hypervelocity collisions of binary stars at the Galactic Centre. MNRAS, 376:492496, Apr. 2007. 
153 O. Y. Gnedin, W. R. Brown, M. J. Geller, and S. J. Kenyon. The Mass Profile of the Galaxy to $80 \mathrm{kpc}$. ApJ, 720:L108-L112, Sept. 2010.

154 O. Y. Gnedin, A. Gould, J. Miralda-Escudé, and A. R. Zentner. Probing the Shape of the Galactic Halo with Hypervelocity Stars. ApJ, 634:344-350, Nov. 2005.

155 F. A. Gómez, G. Besla, D. D. Carpintero, Á. Villalobos, B. W. O'Shea, and E. F. Bell. And Yet it Moves: The Dangers of Artificially Fixing the Milky Way Center of Mass in the Presence of a Massive Large Magellanic Cloud. ApJ, 802(2):128, Apr 2015.

156 R. E. González, A. V. Kravtsov, and N. Y. Gnedin. Satellites in Milky-Way-like Hosts: Environment Dependence and Close Pairs. ApJ, 770:96, June 2013.

157 R. J. J. Grand, A. J. Deason, S. D. M. White, C. M. Simpson, F. A. Gómez, F. Marinacci, and R. Pakmor. The effects of dynamical substructure on Milky Way mass estimates from the high-velocity tail of the local stellar halo. MNRAS, 487(1):L72-L76, Jul 2019.

158 R. J. J. Grand, F. A. Gómez, F. Marinacci, R. Pakmor, V. Springel, D. J. R. Campbell, C. S. Frenk, A. Jenkins, and S. D. M. White. The Auriga Project: the properties and formation mechanisms of disc galaxies across cosmic time. MNRAS, 467:179-207, May 2017.

159 R. J. J. Grand, J. Helly, A. Fattahi, M. Cautun, S. Cole, A. P. Cooper, A. J. Deason, C. Frenk, and et al. Aurigaia: mock Gaia DR2 stellar catalogues from the AURIGA cosmological simulations. MNRAS, 481(2):1726-1743, Dec 2018.

160 C. J. Grillmair. Detection of a 60deg-long Dwarf Galaxy Debris Stream. ApJ, 645:L37-L40, July 2006.

161 C. J. Grillmair. Four New Stellar Debris Streams in the Galactic Halo. ApJ, 693:1118-1127, Mar. 2009.

162 C. J. Grillmair. Two New Halo Debris Streams in the Sloan Digital Sky Survey. ApJ, 790:L10, July 2014.

163 C. J. Grillmair, R. Cutri, F. J. Masci, T. Conrow, B. Sesar, P. R. M. Eisenhardt, and E. L. Wright. Detection of a Nearby Halo Debris Stream in the WISE and 2MASS Surveys. ApJ, 769:L23, June 2013.

164 C. J. Grillmair and O. Dionatos. A 22deg Tidal Tail for Palomar 5. ApJ, 641:L37-L39, Apr. 2006.

165 C. J. Grillmair and O. Dionatos. Detection of a 63deg Cold Stellar Stream in the Sloan Digital Sky Survey. ApJ, 643:L17-L20, May 2006.
166 C. J. Grillmair, K. C. Freeman, M. Irwin, and P. J. Quinn. Globular Clusters with Tidal Tails: Deep TwoColor Star Counts. AJ, 109:2553, June 1995.

167 C. J. Grillmair and R. Johnson. The Detection of a 45deg Tidal Stream Associated with the Globular Cluster NGC 5466. ApJ, 639:L17-L20, Mar. 2006.

168 J. E. Gunn, G. R. Knapp, and S. D. Tremaine. The global properties of the Galaxy. II - The Galactic rotation parameters from $21-\mathrm{cm} \mathrm{H} \mathrm{I}$ observations. AJ, 84:1181-1188, Aug. 1979.

169 Q. Guo, S. White, M. Boylan-Kolchin, G. De Lucia, G. Kauffmann, G. Lemson, C. Li, V. Springel, and S. Weinmann. From dwarf spheroidals to cD galaxies: Simulating the galaxy population in a LambdaCDM cosmology. MNRAS, 413:101-131, May 2011.

170 A. Gupta, S. Mathur, Y. Krongold, F. Nicastro, and M. Galeazzi. A Huge Reservoir of Ionized Gas around the Milky Way: Accounting for the Missing Mass? ApJ, 756:L8, Sept. 2012.

171 J. Han, W. Wang, S. Cole, and C. S. Frenk. The orbital PDF: general inference of the gravitational potential from steady-state tracers. MNRAS, 456:10031016, Feb. 2016.

172 J. Han, W. Wang, S. Cole, and C. S. Frenk. The orbital PDF: the dynamical state of Milky Way sized haloes and the intrinsic uncertainty in the determination of their masses. MNRAS, 456:1017-1029, Feb. 2016.

173 J. Han, W. Wang, and Z. Li. Satellite galaxies as better tracers of the Milky Way halo mass. arXiv e-prints, page arXiv:1909.02690, Sep 2019.

174 W. E. Harris. A Catalog of Parameters for Globular Clusters in the Milky Way. AJ, 112:1487, Oct. 1996.

175 W. E. Harris. A New Catalog of Globular Clusters in the Milky Way. arXiv e-prints, Dec. 2010.

176 K. Hattori, M. Valluri, E. F. Bell, and I. U. Roederer. Old, Metal-poor Extreme Velocity Stars in the Solar Neighborhood. ApJ, 866:121, Oct. 2018.

177 K. Hawkins and R. F. G. Wyse. The fastest travel together: chemical tagging of the fastest stars in Gaia DR2 to the stellar halo. MNRAS, 481:1028-1040, Nov. 2018.

178 A. Helmi. Velocity Trends in the Debris of Sagittarius and the Shape of the Dark Matter Halo of Our Galaxy. ApJ, 610:L97-L100, Aug. 2004. 
179 A. Helmi, C. Babusiaux, H. H. Koppelman, D. Massari, J. Veljanoski, and A. G. A. Brown. The merger that led to the formation of the Milky Way's inner stellar halo and thick disk. Nature, 563(7729):85-88, Nov 2018.

180 A. Helmi, S. D. M. White, P. T. de Zeeuw, and H. Zhao. Debris streams in the solar neighbourhood as relicts from the formation of the Milky Way. Nature, 402:5355, Nov. 1999.

181 D. Hendel, V. Scowcroft, K. V. Johnston, M. A. Fardal, R. P. van der Marel, S. T. Sohn, A. M. Price-Whelan, R. L. Beaton, G. Besla, G. Bono, M.-R. L. Cioni, G. Clementini, J. G. Cohen, M. Fabrizio, W. L. Freedman, A. Garofalo, C. J. Grillmair, N. Kallivayalil, J. A. Kollmeier, D. R. Law, B. F. Madore, S. R. Majewski, M. Marengo, A. J. Monson, J. R. Neeley, D. L. Nidever, G. Pietrzyński, M. Seibert, B. Sesar, H. A. Smith, I. Soszyński, and A. Udalski. SMHASH: anatomy of the Orphan Stream using RR Lyrae stars. MNRAS, 479(1):570-587, Sep 2018.

182 M. Henon. L'amas isochrone: I. Annales d'Astrophysique, 22:126, Feb. 1959.

183 J. G. Hills. Hyper-velocity and tidal stars from binaries disrupted by a massive Galactic black hole. Nature, 331:687-689, Feb. 1988.

184 J. Holmberg and C. Flynn. The local density of matter mapped by Hipparcos. MNRAS, 313:209-216, Apr. 2000.

185 J. Holmberg and C. Flynn. The local surface density of disc matter mapped by Hipparcos. MNRAS, 352:440 446, Aug. 2004.

186 D. Homma, M. Chiba, Y. Komiyama, M. Tanaka, S. Okamoto, M. Tanaka, M. N. Ishigaki, K. Hayashi, N. Arimoto, S. G. Carlsten, R. H. Lupton, M. A. Strauss, S. Miyazaki, G. Torrealba, S.-Y. Wang, and H. Murayama. Boötes. IV. A new Milky Way satellite discovered in the Subaru Hyper Suprime-Cam Survey and implications for the missing satellite problem. PASJ, 71(5):94, Oct 2019.

187 D. Homma, M. Chiba, S. Okamoto, Y. Komiyama, M. Tanaka, M. Tanaka, M. N. Ishigaki, K. Hayashi, N. Arimoto, J. A. Garmilla, R. H. Lupton, M. A. Strauss, S. Miyazaki, S.-Y. Wang, and H. Murayama. Searches for new Milky Way satellites from the first two years of data of the Subaru/Hyper Suprime-Cam survey: Discovery of Cetus III. PASJ, 70:S18, Jan 2018.
188 M. Honma, T. Nagayama, K. Ando, T. Bushimata, Y. K. Choi, T. Handa, T. Hirota, H. Imai, and et al. Fundamental Parameters of the Milky Way Galaxy Based on VLBI astrometry. PASJ, 64:136, Dec. 2012.

189 Y. Huang, X.-W. Liu, H.-B. Yuan, M.-S. Xiang, H.W. Zhang, B.-Q. Chen, J.-J. Ren, C. Wang, and et al. The Milky Way's rotation curve out to $100 \mathrm{kpc}$ and its constraint on the Galactic mass distribution. MNRAS, 463:2623-2639, Dec. 2016.

190 A. Irrgang, S. Kreuzer, and U. Heber. Hypervelocity stars in the Gaia era. Runaway B stars beyond the velocity limit of classical ejection mechanisms. A\&A, 620:A48, Dec. 2018.

191 A. Irrgang, B. Wilcox, E. Tucker, and L. Schiefelbein. Milky Way mass models for orbit calculations. A\&A, 549:A137, Jan. 2013.

192 Ž. Ivezić, S. M. Kahn, J. A. Tyson, B. Abel, E. Acosta, R. Allsman, D. Alonso, Y. AlSayyad, and et al. LSST: from Science Drivers to Reference Design and Anticipated Data Products. ArXiv e-prints, May 2008.

193 B. Jalali, F. I. Pelupessy, A. Eckart, S. Portegies Zwart, N. Sabha, A. Borkar, J. Moultaka, K. Mužić, and L. Moser. Star formation in the vicinity of nuclear black holes: young stellar objects close to Sgr A*. MNRAS, 444:1205-1220, Oct. 2014.

194 Y. P. Jing and Y. Suto. Triaxial Modeling of Halo Density Profiles with High-Resolution N-Body Simulations. ApJ, 574:538-553, Aug. 2002.

195 K. V. Johnston, D. R. Law, and S. R. Majewski. A Two Micron All Sky Survey View of the Sagittarius Dwarf Galaxy. III. Constraints on the Flattening of the Galactic Halo. ApJ, 619:800-806, Feb. 2005.

196 E. Kado-Fong, J. E. Greene, J. P. Greco, R. Beaton, A. D. Goulding, S. D. Johnson, and Y. Komiyama. Star Formation in Isolated Dwarf Galaxies Hosting Tidal Debris: Extending the Dwarf-Dwarf Merger Sequence. arXiv e-prints, page arXiv:1911.08497, Nov 2019.

197 P. R. Kafle, S. Sharma, G. F. Lewis, and J. BlandHawthorn. Kinematics of the Stellar Halo and the Mass Distribution of the Milky Way Using Blue Horizontal Branch Stars. ApJ, 761:98, Dec. 2012.

198 P. R. Kafle, S. Sharma, G. F. Lewis, and J. BlandHawthorn. On the Shoulders of Giants: Properties of the Stellar Halo and the Milky Way Mass Distribution. ApJ, 794:59, Oct. 2014. 
199 P. R. Kafle, S. Sharma, A. S. G. Robotham, P. J. Elahi, and S. P. Driver. Jeans that fit: weighing the mass of the Milky Way analogues in the $\Lambda$ CDM universe. MNRAS, 475:4434-4449, Apr. 2018.

200 F. D. Kahn and L. Woltjer. Intergalactic Matter and the Galaxy. ApJ, 130:705, Nov. 1959.

201 N. Kaiser, W. Burgett, K. Chambers, L. Denneau, J. Heasley, R. Jedicke, E. Magnier, J. Morgan, P. Onaka, and J. Tonry. The Pan-STARRS wide-field optical/NIR imaging survey. In Ground-based and Airborne Telescopes III, volume 7733 of Proc. SPIE, page 77330E, July 2010.

202 N. Kallivayalil, L. V. Sales, P. Zivick, T. K. Fritz, A. Del Pino, S. T. Sohn, G. Besla, R. P. van der Marel, and et al. The Missing Satellites of the Magellanic Clouds? Gaia Proper Motions of the Recently Discovered Ultra-faint Galaxies. ApJ, 867(1):19, Nov 2018.

203 N. Kallivayalil, R. P. van der Marel, and C. Alcock. Is the SMC Bound to the LMC? The Hubble Space Telescope Proper Motion of the SMC. ApJ, 652:1213-1229, Dec. 2006.

204 N. Kallivayalil, R. P. van der Marel, C. Alcock, T. Axelrod, K. H. Cook, A. J. Drake, and M. Geha. The Proper Motion of the Large Magellanic Cloud Using HST. ApJ, 638:772-785, Feb. 2006.

205 R. Kennedy, C. Frenk, S. Cole, and A. Benson. Constraining the warm dark matter particle mass with Milky Way satellites. MNRAS, 442(3):2487-2495, Aug 2014.

206 S. J. Kenyon, B. C. Bromley, M. J. Geller, and W. R. Brown. Hypervelocity Stars: From the Galactic Center to the Halo. ApJ, 680:312-327, June 2008.

207 A. A. Kepley, H. L. Morrison, A. Helmi, T. D. Kinman, J. Van Duyne, J. C. Martin, P. Harding, J. E. Norris, and K. C. Freeman. Halo Star Streams in the Solar Neighborhood. AJ, 134(4):1579-1595, Oct 2007.

208 C. King, III, W. R. Brown, M. J. Geller, and S. J. Kenyon. Stellar Velocity Dispersion and Anisotropy of the Milky Way Inner Halo. ApJ, 813:89, Nov. 2015.

209 A. Klypin, A. V. Kravtsov, O. Valenzuela, and F. Prada. Where Are the Missing Galactic Satellites? ApJ, 522(1):82-92, Sep 1999.

210 A. Klypin, H. Zhao, and R. S. Somerville. $\Lambda$ CDMbased Models for the Milky Way and M31. I. Dynamical Models. ApJ, 573:597-613, July 2002.
211 A. A. Klypin, S. Trujillo-Gomez, and J. Primack. Dark Matter Halos in the Standard Cosmological Model: Results from the Bolshoi Simulation. ApJ, 740:102, Oct. 2011.

212 S. Kobayashi, Y. Hainick, R. Sari, and E. M. Rossi. Ejection and Capture Dynamics in Restricted Threebody Encounters. ApJ, 748:105, Apr. 2012.

213 C. S. Kochanek. The Mass of the Milky Way. ApJ, 457:228, Jan. 1996.

214 J. A. Kollmeier, G. Zasowski, H.-W. Rix, M. Johns, S. F. Anderson, N. Drory, J. A. Johnson, R. W. Pogge, and et al. SDSS-V: Pioneering Panoptic Spectroscopy. arXiv e-prints, page arXiv:1711.03234, Nov 2017.

215 S. E. Koposov, V. Belokurov, T. S. Li, C. Mateu, D. Erkal, C. J. Grillmair, D. Hendel, A. M. PriceWhelan, C. F. P. Laporte, K. Hawkins, S. T. Sohn, A. del Pino, N. W. Evans, C. T. Slater, N. Kallivayalil, J. F. Navarro, and Orphan Aspen Treasury Collaboration. Piercing the Milky Way: an all-sky view of the Orphan Stream. MNRAS, 485(4):4726-4742, Jun 2019.

216 S. E. Koposov, M. Irwin, V. Belokurov, E. GonzalezSolares, A. K. Yoldas, J. Lewis, N. Metcalfe, and T. Shanks. Discovery of a cold stellar stream in the ATLAS DR1 data. MNRAS, 442:L85-L89, July 2014.

217 S. E. Koposov, H.-W. Rix, and D. W. Hogg. Constraining the Milky Way Potential with a Six-Dimensional Phase-Space Map of the GD-1 Stellar Stream. ApJ, 712:260-273, Mar. 2010.

218 K. Kuijken and G. Gilmore. The Mass Distribution in the Galactic Disc - II - Determination of the Surface Mass Density of the Galactic Disc Near the Sun. MNRAS, 239:605-649, Aug. 1989.

219 K. Kuijken and G. Gilmore. The galactic disk surface mass density and the Galactic force $\mathrm{K}(\mathrm{z})$ at $\mathrm{Z}=1.1$ kiloparsecs. ApJ, 367:L9-L13, Jan. 1991.

220 A. S. Kulessa and D. Lynden-Bell. The mass of the Milky Way Galaxy. MNRAS, 255:105-118, Mar. 1992.

221 A. Kunder, G. Kordopatis, M. Steinmetz, T. Zwitter, P. J. McMillan, L. Casagrande, H. Enke, J. Wojno, and et al. The Radial Velocity Experiment (RAVE): Fifth Data Release. AJ, 153:75, Feb. 2017.

222 A. H. W. Küpper, E. Balbinot, A. Bonaca, K. V. Johnston, D. W. Hogg, P. Kroupa, and B. X. Santiago. Globular Cluster Streams as Galactic High-Precision Scalesthe Poster Child Palomar 5. ApJ, 803:80, Apr. 2015. 
223 A. H. W. Küpper, R. R. Lane, and D. C. Heggie. More on the structure of tidal tails. MNRAS, 420:2700-2714, Mar. 2012.

224 C. F. P. Laporte, K. V. Johnston, F. A. Gómez, N. Garavito-Camargo, and G. Besla. The influence of Sagittarius and the Large Magellanic Cloud on the stellar disc of the Milky Way Galaxy. MNRAS, 481(1):286-306, Nov 2018.

225 C. F. P. Laporte, K. V. Johnston, and A. Tzanidakis. Stellar disc streams as probes of the Galactic potential and satellite impacts. MNRAS, 483(2):1427-1436, Feb 2019.

226 D. R. Law, K. V. Johnston, and S. R. Majewski. A Two Micron All-Sky Survey View of the Sagittarius Dwarf Galaxy. IV. Modeling the Sagittarius Tidal Tails. ApJ, 619:807-823, Feb. 2005.

227 D. R. Law and S. R. Majewski. The Sagittarius Dwarf Galaxy: A Model for Evolution in a Triaxial Milky Way Halo. ApJ, 714:229-254, May 2010.

228 K. H. Lee, H. M. Lee, G. G. Fahlman, and M. G. Lee. Wide-Field CCD Photometry of the Globular Cluster M92. AJ, 126:815-825, Aug. 2003.

229 I. Lehmann and R.-D. Scholz. Tidal radii of the globular clusters M 5, M 12, M 13, M 15, M 53, NGC 5053 and NGC 5466 from automated star counts. A\&A, 320:776-782, Apr. 1997.

230 S. Leon, G. Meylan, and F. Combes. Tidal tails around 20 Galactic globular clusters. Observational evidence for gravitational disk/bulge shocking. A\&A, 359:907931, July 2000.

231 P. J. T. Leonard and S. Tremaine. The local Galactic escape speed. ApJ, 353:486-493, Apr. 1990.

232 E. S. Levine, C. Heiles, and L. Blitz. The Milky Way Rotation Curve and Its Vertical Derivatives: Inside the Solar Circle. ApJ, 679:1288-1298, June 2008.

233 Y.-S. Li and S. D. M. White. Masses for the Local Group and the Milky Way. MNRAS, 384:1459-1468, Mar. 2008.

234 Z.-Z. Li, Y. P. Jing, Y.-Z. Qian, Z. Yuan, and D.-H. Zhao. Determination of Dark Matter Halo Mass from Dynamics of Satellite Galaxies. ApJ, 850:116, Dec. 2017.
235 Z.-Z. Li, Y.-Z. Qian, J. Han, T. S. Li, W. Wang, and Y. P. Jing. Constraining the milky way mass profile with phase-space distribution of satellite galaxies. 2019.

236 Z.-Z. Li, Y.-Z. Qian, J. Han, W. Wang, and Y. P. Jing. A Versatile and Accurate Method for Halo Mass Determination from Phase-space Distribution of Satellite Galaxies. ApJ, 886(1):69, Nov 2019.

237 D. N. Limber and W. G. Mathews. The Dynamical Stability of Stephan's Quintet. ApJ, 132:286, Sept. 1960

238 D. N. C. Lin, B. F. Jones, and A. R. Klemola. The motion of the Magellanic clouds, origin of the Magellanic Stream, and the mass of the Milky Way. ApJ, 439:652671, Feb. 1995.

239 B. Little and S. Tremaine. Distant satellites as probes of our Galaxy's mass distribution. ApJ, 320:493-501, Sept. 1987.

240 L. Liu, B. F. Gerke, R. H. Wechsler, P. S. Behroozi, and M. T. Busha. How Common are the Magellanic Clouds? ApJ, 733:62, May 2011.

241 S. R. Loebman, Ž. Ivezić, T. R. Quinn, J. Bovy, C. R. Christensen, M. Jurić, R. Roškar, A. M. Brooks, and F. Governato. The Milky Way Tomography with Sloan Digital Sky Survey. V. Mapping the Dark Matter Halo. ApJ, 794(2):151, Oct 2014

242 S. R. Loebman, M. Valluri, K. Hattori, V. P. Debattista, E. F. Bell, G. Stinson, C. R. Christensen, A. Brooks, and et al. Beta Dips in the Gaia Era: Simulation Predictions of the Galactic Velocity Anisotropy Parameter $(\beta)$ for Stellar Halos. ApJ, 853(2):196, Feb 2018.

243 B. Lowing, W. Wang, A. Cooper, R. Kennedy, J. Helly, S. Cole, and C. Frenk. Creating mock catalogues of stellar haloes from cosmological simulations. MNRAS, 446:2274-2290, Jan. 2015.

244 J. R. Lu. Massive Young Clusters Near the Galactic Center. In S. Stahler, editor, The Birth of Star Clusters, volume 424 of Astrophysics and Space Science Library, page 69, 2018.

245 A. L. Luo, Y.-H. Zhao, G. Zhao, L.-C. Deng, X.-W. Liu, Y.-P. Jing, G. Wang, and H.-T. Zhang. The first data release (DR1) of the LAMOST regular survey. Research in Astronomy and Astrophysics, 15(8):1095, Aug 2015.

246 H. Lux, J. I. Read, G. Lake, and K. V. Johnston. NGC 5466: a unique probe of the Galactic halo shape. MNRAS, 424:L16-L20, July 2012. 
247 H. Lux, J. I. Read, G. Lake, and K. V. Johnston. Constraining the Milky Way halo shape using thin streams. MNRAS, 436:2386-2397, Dec. 2013.

248 D. Lynden-Bell. Stellar dynamics. Only isolating integrals should be used in Jeans theorem. MNRAS, 124:1, 1962.

249 A. V. Macciò and F. Fontanot. How cold is dark matter? Constraints from Milky Way satellites. MNRAS, 404(1):L16-L20, May 2010.

250 J. Magorrian. Bayes versus the virial theorem: inferring the potential of a galaxy from a kinematical snapshot. MNRAS, 437:2230-2248, Jan. 2014.

251 K. Malhan and R. A. Ibata. Constraining the Milky Way halo potential with the GD-1 stellar stream. MNRAS, 486(3):2995-3005, Jul 2019.

252 T. Marchetti, O. Contigiani, E. M. Rossi, J. G. Albert, A. G. A. Brown, and A. Sesana. Predicting the hypervelocity star population in Gaia. MNRAS, 476:46974712, June 2018.

253 T. Marchetti, E. M. Rossi, and A. G. A. Brown. Gaia DR2 in 6D: Searching for the fastest stars in the Galaxy. MNRAS, Sept. 2018.

254 S. L. Martell, S. Sharma, S. Buder, L. Duong, K. J. Schlesinger, J. Simpson, K. Lind, M. Ness, and et al. The GALAH survey: observational overview and Gaia DR1 companion. MNRAS, 465:3203-3219, Mar. 2017.

255 N. F. Martin, R. A. Ibata, R. M. Rich, M. L. M. Collins, M. A. Fardal, M. J. Irwin, G. F. Lewis, A. W. McConnachie, and et al. The PAndAS Field of Streams: Stellar Structures in the Milky Way Halo toward Andromeda and Triangulum. ApJ, 787:19, May 2014.

256 D. Martínez-Delgado, R. J. Gabany, K. Crawford, S. Zibetti, S. R. Majewski, H.-W. Rix, J. Fliri, J. A. CarballoBello, and et al. Stellar Tidal Streams in Spiral Galaxies of the Local Volume: A Pilot Survey with Modest Aperture Telescopes. AJ, 140:962-967, Oct. 2010.

257 D. Martínez-Delgado, M. Á. Gómez-Flechoso, A. Aparicio, and R. Carrera. Tracing Out the Northern Tidal Stream of the Sagittarius Dwarf Spheroidal Galaxy. ApJ, 601:242-259, Jan. 2004.

258 D. Massari and A. Helmi. With and without spectroscopy: Gaia DR2 proper motions of seven ultra-faint dwarf galaxies. A\&A, 620:A155, Dec 2018.
259 A. W. McConnachie. The Observed Properties of Dwarf Galaxies in and around the Local Group. AJ, 144:4, July 2012.

260 A. W. McConnachie, M. J. Irwin, R. A. Ibata, J. Dubinski, L. M. Widrow, N. F. Martin, P. Côté, A. L. Dotter, and et al. The remnants of galaxy formation from a panoramic survey of the region around M31. Nature, 461:66-69, Sept. 2009.

261 P. J. McMillan. Mass models of the Milky Way. MNRAS, 414:2446-2457, July 2011.

262 P. J. McMillan. The mass distribution and gravitational potential of the Milky Way. MNRAS, 465:76-94, Feb. 2017.

263 P. J. McMillan and J. J. Binney. The uncertainty in Galactic parameters. MNRAS, 402:934-940, Feb. 2010 .

264 M. J. Miller and J. N. Bregman. Constraining the Milky Way's Hot Gas Halo with O VII and O VIII Emission Lines. ApJ, 800:14, Feb. 2015.

265 G. Monari, B. Famaey, I. Carrillo, T. Piffl, M. Steinmetz, R. F. G. Wyse, F. Anders, C. Chiappini, and K. Janßen. The escape speed curve of the Galaxy obtained from Gaia DR2 implies a heavy Milky Way. A\&A, 616:L9, Aug. 2018.

266 G. Monari, B. Famaey, and A. Siebert. Modelling the Galactic disc: perturbed distribution functions in the presence of spiral arms. MNRAS, 457:2569-2582, Apr. 2016.

267 B. Moore, S. Ghigna, F. Governato, G. Lake, T. Quinn, J. Stadel, and P. Tozzi. Dark Matter Substructure within Galactic Halos. ApJ, 524(1):L19-L22, Oct 1999.

268 Y. Morinaga, T. Ishiyama, T. Kirihara, and K. Kinjo. Statistical properties of substructures around Milky Way-sized haloes and their implications for the formation of stellar streams. MNRAS, 487(2):2718-2729, Aug 2019.

269 H. L. Morrison, M. Mateo, E. W. Olszewski, P. Harding, R. C. Dohm-Palmer, K. C. Freeman, J. E. Norris, and M. Morita. Mapping the Galactic Halo. I. The “Spaghetti” Survey. AJ, 119:2254-2273, May 2000.

270 T. Murai and M. Fujimoto. The Magellanic Stream and the Galaxy with a Massive Halo. PASJ, 32:581, 1980. 
271 G. C. Myeong, N. W. Evans, V. Belokurov, N. C. Amorisco, and S. E. Koposov. Halo substructure in the SDSS-Gaia catalogue: streams and clumps. MNRAS, 475:1537-1548, Apr. 2018.

272 G. C. Myeong, N. W. Evans, V. Belokurov, J. L. Sanders, and S. E. Koposov. Discovery of new retrograde substructures: the shards of $\omega$ Centauri? MNRAS, 478:5449-5459, Aug. 2018.

273 G. C. Myeong, N. W. Evans, V. Belokurov, J. L. Sanders, and S. E. Koposov. The Milky Way Halo in Action Space. ApJ, 856:L26, Apr. 2018.

274 G. C. Myeong, H. Jerjen, D. Mackey, and G. S. Da Costa. Tidal Tails around the Outer Halo Globular Clusters Eridanus and Palomar 15. ApJ, 840:L25, May 2017.

275 J. F. Navarro, C. S. Frenk, and S. D. M. White. The Structure of Cold Dark Matter Halos. ApJ, 462:563, May 1996.

276 J. F. Navarro, C. S. Frenk, and S. D. M. White. A Universal Density Profile from Hierarchical Clustering. ApJ, 490:493-508, Dec. 1997.

277 S. Nayakshin and K. Zubovas. Sgr A* envelope explosion and the young stars in the centre of the Milky Way. MNRAS, 478:L127-L131, July 2018.

278 D. Nelson, A. Pillepich, S. Genel, M. Vogelsberger, V. Springel, P. Torrey, V. Rodriguez-Gomez, D. Sijacki, G. F. Snyder, and et al. The illustris simulation: Public data release. Astronomy and Computing, 13:12-37, Nov. 2015.

279 F. Nesti and P. Salucci. The Dark Matter halo of the Milky Way, AD 2013. J. Cosmology Astropart. Phys., 7:016, July 2013.

280 H. J. Newberg, B. A. Willett, B. Yanny, and Y. Xu. The Orbit of the Orphan Stream. ApJ, 711:32-49, Mar. 2010.

281 H. J. Newberg, B. Yanny, and B. A. Willett. Discovery of a New, Polar-Orbiting Debris Stream in the Milky Way Stellar Halo. ApJ, 700:L61-L64, Aug. 2009.

282 O. Newton, M. Cautun, A. Jenkins, C. S. Frenk, and J. C. Helly. The total satellite population of the Milky Way. MNRAS, 479(3):2853-2870, Sep 2018.

283 F. Nicastro, F. Senatore, Y. Krongold, S. Mathur, and M. Elvis. A Distant Echo of Milky Way Central Activity Closes the Galaxy's Baryon Census. ApJ, 828:L12, Sept. 2016.
284 J. E. Norris and M. R. S. Hawkins. Population studies. $\mathrm{X}$ - Constraints on the mass and extent of the Galaxy's dark corona. ApJ, 380:104-115, Oct. 1991.

285 M. Odenkirchen, E. K. Grebel, W. Dehnen, H.-W. Rix, B. Yanny, H. J. Newberg, C. M. Rockosi, D. MartínezDelgado, and et al. The Extended Tails of Palomar 5: A 10deg Arc of Globular Cluster Tidal Debris. AJ, 126:2385-2407, Nov. 2003.

286 M. Odenkirchen, E. K. Grebel, C. M. Rockosi, W. Dehnen, R. Ibata, H.-W. Rix, A. Stolte, C. Wolf, and et al. Detection of Massive Tidal Tails around the Globular Cluster Palomar 5 with Sloan Digital Sky Survey Commissioning Data. ApJ, 548:L165-L169, Feb. 2001.

287 R. M. O’Leary and A. Loeb. Production of hypervelocity stars through encounters with stellar-mass black holes in the Galactic Centre. MNRAS, 383:86-92, Jan. 2008.

288 J. H. Oort. Asymmetry in the distribution of stellar velocities. The Observatory, 49:302-304, Oct. 1926.

289 J. H. Oort. Dynamics of the galactic system in the vicinity of the Sun. Bull. Astron. Inst. Netherlands, 4:269, Nov. 1928.

290 A. B. Pace and T. S. Li. Proper Motions of Milky Way Ultra-faint Satellites with Gaia DR2 × DES DR1. ApJ, 875(1):77, Apr 2019.

291 E. Patel, G. Besla, and K. Mandel. Orbits of massive satellite galaxies - II. Bayesian estimates of the Milky Way and Andromeda masses using high-precision astrometry and cosmological simulations. MNRAS, 468:3428-3449, July 2017.

292 E. Patel, G. Besla, K. Mandel, and S. T. Sohn. Estimating the Mass of the Milky Way Using the Ensemble of Classical Satellite Galaxies. ApJ, 857:78, Apr. 2018.

293 E. Patel, G. Besla, and S. T. Sohn. Orbits of massive satellite galaxies - I. A close look at the Large Magellanic Cloud and a new orbital history for M33. MNRAS, 464:3825-3849, Feb. 2017.

294 M. Pato and F. Iocco. galkin: A new compilation of Milky Way rotation curve data. SoftwareX, 6:54-62, Jan 2017. 
295 T. Paumard, R. Genzel, F. Martins, S. Nayakshin, A. M. Beloborodov, Y. Levin, S. Trippe, F. Eisenhauer, and et al. The Two Young Star Disks in the Central Parsec of the Galaxy: Properties, Dynamics, and Formation. ApJ, 643:1011-1035, June 2006.

296 M. S. Pawlowski, J. Pflamm-Altenburg, and P. Kroupa. The VPOS: a vast polar structure of satellite galaxies, globular clusters and streams around the Milky Way. MNRAS, 423:1109-1126, June 2012.

297 J. Peñarrubia and A. Fattahi. What galaxy masses perturb the local cosmic expansion? MNRAS, 468:13001316, June 2017.

298 J. Peñarrubia, F. A. Gómez, G. Besla, D. Erkal, and Y.Z. Ma. A timing constraint on the (total) mass of the Large Magellanic Cloud. MNRAS, 456:L54-L58, Feb. 2016.

299 J. Peñarrubia, Y.-Z. Ma, M. G. Walker, and A. McConnachie. A dynamical model of the local cosmic expansion. MNRAS, 443:2204-2222, Sept. 2014.

300 H. B. Perets, X. Wu, H. S. Zhao, B. Famaey, G. Gentile, and T. Alexander. The Galactic Potential and the Asymmetric Distribution of Hypervelocity Stars. ApJ, 697:2096-2101, June 2009.

301 M. A. C. Perryman, K. S. de Boer, G. Gilmore, E. Høg, M. G. Lattanzi, L. Lindegren, X. Luri, F. Mignard, O. Pace, and P. T. de Zeeuw. GAIA: Composition, formation and evolution of the Galaxy. A\&A, 369:339363, Apr. 2001.

302 M. S. Petersen and J. Peñarrubia. Reflex motion in the Milky Way stellar halo resulting from the Large Magellanic Cloud infall. MNRAS, Feb 2020.

303 S. Piatek, C. Pryor, P. Bristow, E. W. Olszewski, H. C. Harris, M. Mateo, D. Minniti, and C. G. Tinney. Proper Motions of Dwarf Spheroidal Galaxies from Hubble Space Telescope Imaging. III. Measurement for Ursa Minor. AJ, 130:95-115, July 2005.

304 S. Piatek, C. Pryor, P. Bristow, E. W. Olszewski, H. C. Harris, M. Mateo, D. Minniti, and C. G. Tinney. Proper Motions of Dwarf Spheroidal Galaxies from Hubble Space Telescope Imaging. IV. Measurement for Sculptor. AJ, 131:1445-1460, Mar. 2006.

305 S. Piatek, C. Pryor, P. Bristow, E. W. Olszewski, H. C. Harris, M. Mateo, D. Minniti, and C. G. Tinney. Proper Motions of Dwarf Spheroidal Galaxies from Hubble
Space Telescope Imaging. V. Final Measurement for Fornax. AJ, 133:818-844, Mar. 2007.

306 S. Piatek, C. Pryor, and E. Olszewski. Space Motions of the Draco, Fornax, and Sagittarius Dwarf Spheroidal Galaxies. Astrophysics and Space Science Proceedings, 5:199, 2008.

307 S. Piatek, C. Pryor, and E. W. Olszewski. Proper Motions of the Large Magellanic Cloud and Small Magellanic Cloud: Re-Analysis of Hubble Space Telescope Data. AJ, 135:1024-1038, Mar. 2008.

308 S. Piatek, C. Pryor, and E. W. Olszewski. Proper Motion of the Leo II Dwarf Galaxy Based On Hubble Space Telescope Imaging. AJ, 152:166, Dec. 2016.

309 S. Piatek, C. Pryor, E. W. Olszewski, H. C. Harris, M. Mateo, D. Minniti, D. G. Monet, H. Morrison, and C. G. Tinney. Proper Motions of Dwarf Spheroidal Galaxies from Hubble Space Telescope Imaging. I. Method and a Preliminary Measurement for Fornax. AJ, 124:3198-3221, Dec. 2002.

310 S. Piatek, C. Pryor, E. W. Olszewski, H. C. Harris, M. Mateo, D. Minniti, and C. G. Tinney. Proper Motions of Dwarf Spheroidal Galaxies from Hubble Space Telescope Imaging. II. Measurement for Carina. AJ, 126:2346-2361, Nov. 2003.

311 T. Piffl, J. Binney, P. J. McMillan, M. Steinmetz, A. Helmi, R. F. G. Wyse, O. Bienaymé, J. BlandHawthorn, and et al. Constraining the Galaxy's dark halo with RAVE stars. MNRAS, 445:3133-3151, Dec. 2014.

312 T. Piffl, C. Scannapieco, J. Binney, M. Steinmetz, R.D. Scholz, M. E. K. Williams, R. S. de Jong, G. Kordopatis, and et al. The RAVE survey: the Galactic escape speed and the mass of the Milky Way. A\&A, 562:A91, Feb. 2014.

313 B. Pila-Díez, J. T. A. de Jong, K. Kuijken, R. F. J. van der Burg, and H. Hoekstra. A skewer survey of the Galactic halo from deep CFHT and INT images. A\&A, 579:A38, Jul 2015.

314 E. Polisensky and M. Ricotti. Constraints on the dark matter particle mass from the number of Milky Way satellites. Phys. Rev. D, 83(4):043506, Feb 2011.

315 L. Posti, J. Binney, C. Nipoti, and L. Ciotti. Actionbased distribution functions for spheroidal galaxy components. MNRAS, 447:3060-3068, Mar. 2015. 
316 L. Posti and A. Helmi. Mass and shape of the Milky Way's dark matter halo with globular clusters from Gaia and Hubble. A\&A, 621:A56, Jan. 2019.

317 A. M. Price-Whelan, D. W. Hogg, K. V. Johnston, and D. Hendel. Inferring the Gravitational Potential of the Milky Way with a Few Precisely Measured Stars. ApJ, 794:4, Oct. 2014.

318 M. J. Reid and A. Brunthaler. The Proper Motion of Sagittarius A*. II. The Mass of Sagittarius A*. ApJ, 616:872-884, Dec. 2004.

319 M. J. Reid, K. M. Menten, X. W. Zheng, A. Brunthaler, L. Moscadelli, Y. Xu, B. Zhang, M. Sato, and et al. Trigonometric Parallaxes of Massive Star-Forming Regions. VI. Galactic Structure, Fundamental Parameters, and Noncircular Motions. ApJ, 700:137-148, July 2009.

320 R. M. Rich. Spectroscopy and abundances of 88 K giants in Baade's Window. AJ, 95:828-865, Mar. 1988.

321 B. M. Rossetto, B. X. Santiago, L. Girardi, J. I. B. Camargo, E. Balbinot, L. N. da Costa, B. Yanny, M. A. G. Maia, and et al. The Dark Energy Survey: Prospects for Resolved Stellar Populations. AJ, 141:185, June 2011.

322 E. M. Rossi, S. Kobayashi, and R. Sari. The Velocity Distribution of Hypervelocity Stars. ApJ, 795:125, Nov. 2014.

323 E. M. Rossi, T. Marchetti, M. Cacciato, M. Kuiack, and R. Sari. Joint constraints on the Galactic dark matter halo and Galactic Centre from hypervelocity stars. MNRAS, 467:1844-1856, May 2017.

324 T. Sakamoto, M. Chiba, and T. C. Beers. The mass of the Milky Way: Limits from a newly assembled set of halo objects. A\&A, 397:899-911, Jan. 2003.

325 L. V. Sales, J. F. Navarro, M. G. Abadi, and M. Steinmetz. Satellites of simulated galaxies: survival, merging and their relationto the dark and stellar haloes. MNRAS, 379:1464-1474, Aug. 2007.

326 J. Sanders. Angle-action estimation in a general axisymmetric potential. MNRAS, 426:128-139, Oct. 2012.

327 J. L. Sanders. Probabilistic model for constraining the Galactic potential using tidal streams. MNRAS, 443:423-431, Sept. 2014.
328 J. L. Sanders and J. Binney. Stream-orbit misalignment - I. The dangers of orbit-fitting. MNRAS, 433:18131825, Aug. 2013.

329 J. L. Sanders and J. Binney. Stream-orbit misalignment - II. A new algorithm to constrain the Galactic potential. MNRAS, 433:1826-1836, Aug. 2013.

330 J. L. Sanders and J. Binney. A fast algorithm for estimating actions in triaxial potentials. MNRAS, 447:2479-2496, Mar. 2015.

331 J. L. Sanders and J. Binney. Extended distribution functions for our Galaxy. MNRAS, 449:3479-3502, June 2015.

332 J. L. Sanders and J. Binney. TACT: The Action Computation Tool. Astrophysics Source Code Library, Dec. 2015.

333 J. L. Sanders and J. Binney. A review of action estimation methods for galactic dynamics. MNRAS, 457:2107-2121, Apr. 2016.

334 J. L. Sanders, J. Bovy, and D. Erkal. Dynamics of stream-subhalo interactions. MNRAS, 457:3817-3835, Apr. 2016.

335 R. E. Sanderson, J. Hartke, and A. Helmi. Modeling the Gravitational Potential of a Cosmological Dark Matter Halo with Stellar Streams. ApJ, 836:234, Feb. 2017.

336 R. Sari, S. Kobayashi, and E. M. Rossi. Hypervelocity Stars and the Restricted Parabolic Three-Body Problem. ApJ, 708:605-614, Jan. 2010.

337 T. Sawala, C. S. Frenk, A. Fattahi, J. F. Navarro, R. G. Bower, R. A. Crain, C. Dalla Vecchia, M. Furlong, and et al. The APOSTLE simulations: solutions to the Local Group's cosmic puzzles. MNRAS, 457:1931-1943, Apr. 2016.

338 J. Schaye, R. A. Crain, R. G. Bower, M. Furlong, M. Schaller, T. Theuns, C. Dalla Vecchia, C. S. Frenk, and et al. The EAGLE project: simulating the evolution and assembly of galaxies and their environments. MNRAS, 446:521-554, Jan. 2015.

339 R. Schönrich. Galactic rotation and solar motion from stellar kinematics. MNRAS, 427:274-287, Nov. 2012.

340 R. Schönrich and M. Aumer. Assessing distances and consistency of kinematics in Gaia/TGAS. MNRAS, 472(4):3979-3998, Dec 2017.

341 R. Schönrich and J. Binney. A new formula for disc kinematics. MNRAS, 419:1546-1556, Jan. 2012. 
342 M. Schwarzschild. A numerical model for a triaxial stellar system in dynamical equilibrium. ApJ, 232:236247, Aug. 1979.

343 A. Sesana, F. Haardt, and P. Madau. Hypervelocity stars and the environment of Sgr A*. MNRAS, 379:L45L49, July 2007.

344 B. Sesar, C. J. Grillmair, J. G. Cohen, E. C. Bellm, V. B. Bhalerao, D. Levitan, R. R. Laher, E. O. Ofek, and et al. Tracing the Orphan Stream to $55 \mathrm{kpc}$ with RR Lyrae Stars. ApJ, 776:26, Oct. 2013.

345 B. Sesar, Ž. Ivezić, J. S. Stuart, D. M. Morgan, A. C. Becker, S. Sharma, L. Palaversa, M. Jurić, P. Wozniak, and H. Oluseyi. Exploring the Variable Sky with LINEAR. II. Halo Structure and Substructure Traced by RR Lyrae Stars to $30 \mathrm{kpc}$. AJ, 146:21, Aug. 2013.

346 B. Sesar, M. Jurić, and Ž. Ivezić. The Shape and Profile of the Milky Way Halo as Seen by the CanadaFrance-Hawaii Telescope Legacy Survey. ApJ, 731:4, Apr. 2011.

347 T. Shanks, V. Belokurov, B. Chehade, S. M. Croom, J. R. Findlay, E. Gonzalez-Solares, M. J. Irwin, S. Koposov, and et al. VST ATLAS First Science Results. The Messenger, 154:38-40, Dec. 2013.

348 S. Shao, M. Cautun, A. J. Deason, C. S. Frenk, and T. Theuns. Evolution of LMC/M33-mass dwarf galaxies in the EAGLE simulation. MNRAS, 479(1):284296, Sep 2018.

349 S. Shao, M. Cautun, and C. S. Frenk. Evolution of galactic planes of satellites in the EAGLE simulation. MNRAS, 488(1):1166-1179, Sep 2019.

350 N. Shipp, A. Drlica-Wagner, E. Balbinot, P. Ferguson, D. Erkal, T. S. Li, K. Bechtol, V. Belokurov, and et al. Stellar Streams Discovered in the Dark Energy Survey. ApJ, 862:114, Aug. 2018.

351 A. Siebert, O. Bienaymé, and C. Soubiran. Vertical distribution of Galactic disk stars. II. The surface mass density in the Galactic plane. A\&A, 399:531-541, Feb 2003.

352 J. D. Simon. Gaia Proper Motions and Orbits of the Ultra-faint Milky Way Satellites. ApJ, 863(1):89, Aug 2018.

353 J. D. Simon. The Faintest Dwarf Galaxies. ARA\&A, 57:375-415, Aug 2019.
354 E. Sirko, J. Goodman, G. R. Knapp, J. Brinkmann, Ž. Ivezić, E. J. Knerr, D. Schlegel, D. P. Schneider, and D. G. York. Blue Horizontal-Branch Stars in the Sloan Digital Sky Survey. II. Kinematics of the Galactic Halo. AJ, 127:914-924, Feb. 2004.

355 M. F. Skrutskie, R. M. Cutri, R. Stiening, M. D. Weinberg, S. Schneider, J. M. Carpenter, C. Beichman, R. Capps, and et al. The Two Micron All Sky Survey (2MASS). AJ, 131:1163-1183, Feb. 2006.

356 M. C. Smith, N. W. Evans, V. Belokurov, P. C. Hewett, D. M. Bramich, G. Gilmore, M. J. Irwin, S. Vidrih, and D. B. Zucker. Kinematics of SDSS subdwarfs: structure and substructure of the Milky Way halo. MNRAS, 399:1223-1237, Nov. 2009.

357 M. C. Smith, G. R. Ruchti, A. Helmi, R. F. G. Wyse, J. P. Fulbright, K. C. Freeman, J. F. Navarro, G. M. Seabroke, and et al. The RAVE survey: constraining the local Galactic escape speed. MNRAS, 379:755772, Aug. 2007.

358 M. C. Smith, S. H. Whiteoak, and N. W. Evans. Slicing and Dicing the Milky Way Disk in the Sloan Digital Sky Survey. ApJ, 746:181, Feb. 2012.

359 Y. Sofue. CO-Line Rotation Curves, Deep Potential of Massive Cores, and High-density Molecular Nuclei. Astrophysics and Space Science Proceedings, 4:178, Jan 2008.

360 S. T. Sohn, J. Anderson, and R. P. van der Marel. The M31 Velocity Vector. I. Hubble Space Telescope Proper-motion Measurements. ApJ, 753:7, July 2012.

361 S. T. Sohn, G. Besla, R. P. van der Marel, M. BoylanKolchin, S. R. Majewski, and J. S. Bullock. The Space Motion of Leo I: Hubble Space Telescope Proper Motion and Implied Orbit. ApJ, 768:139, May 2013.

362 S. T. Sohn, R. P. van der Marel, J. L. Carlin, S. R. Majewski, N. Kallivayalil, D. R. Law, J. Anderson, and M. H. Siegel. Hubble Space Telescope Proper Motions along the Sagittarius Stream. I. Observations and Results for Stars in Four Fields. ApJ, 803:56, Apr. 2015.

363 S. T. Sohn, L. L. Watkins, M. A. Fardal, R. P. van der Marel, A. J. Deason, G. Besla, and A. Bellini. Absolute Hubble Space Telescope Proper Motion (HSTPROMO) of Distant Milky Way Globular Clusters: Galactocentric Space Velocities and the Milky Way Mass. ApJ, 862:52, July 2018. 
364 J. Sommer-Larsen, T. C. Beers, C. Flynn, R. Wilhelm, and P. R. Christensen. A Dynamical and Kinematic Model of the Galactic Stellar Halo and Possible Implications for Galaxy Formation Scenarios. ApJ, 481:775781, May 1997.

365 V. Springel, J. Wang, M. Vogelsberger, A. Ludlow, A. Jenkins, A. Helmi, J. F. Navarro, C. S. Frenk, and S. D. M. White. The Aquarius Project: the subhaloes of galactic haloes. MNRAS, 391:1685-1711, Dec. 2008.

366 V. Springel, S. D. M. White, A. Jenkins, C. S. Frenk, N. Yoshida, L. Gao, J. Navarro, R. Thacker, and et al. Simulations of the formation, evolution and clustering of galaxies and quasars. Nature, 435:629-636, June 2005.

367 S. M. Staudaher, D. A. Dale, L. van Zee, K. L. Barnes, and D. O. Cook. The stellar halo and tidal streams of Messier 63. MNRAS, 454:3613-3621, Dec. 2015.

368 M. Steinmetz, T. Zwitter, A. Siebert, F. G. Watson, K. C. Freeman, U. Munari, R. Campbell, M. Williams, and et al. The Radial Velocity Experiment (RAVE): First Data Release. AJ, 132:1645-1668, Oct. 2006.

369 R. Szabó, K. Kolenberg, L. Molnár, E. Plachy, P. Klagyivik, and O. Hanyecz. The K2 RR Lyrae Survey. In European Physical Journal Web of Conferences, volume 160 of European Physical Journal Web of Conferences, page 04004, Oct. 2017.

370 M. Takada, R. S. Ellis, M. Chiba, J. E. Greene, H. Aihara, N. Arimoto, K. Bundy, J. Cohen, O. Doré, and et al. Extragalactic science, cosmology, and Galactic archaeology with the Subaru Prime Focus Spectrograph. PASJ, 66:R1, Feb. 2014.

371 T. Tal, P. G. van Dokkum, J. Nelan, and R. Bezanson. The Frequency of Tidal Features Associated with Nearby Luminous Elliptical Galaxies From a Statistically Complete Sample. AJ, 138:1417-1427, Nov. 2009.

372 C. Taylor, M. Boylan-Kolchin, P. Torrey, M. Vogelsberger, and L. Hernquist. The mass profile of the Milky Way to the virial radius from the Illustris simulation. MNRAS, 461:3483-3493, Oct. 2016.

373 H.-J. Tian, P. Gupta, B. Sesar, H.-W. Rix, N. F. Martin, C. Liu, B. Goldman, I. Platais, and et al. A Gaia-PS1SDSS (GPS1) Proper Motion Catalog Covering 3/4 of the Sky. ApJS, 232(1):4, Sep 2017.
374 E. J. Tollerud, M. Boylan-Kolchin, E. J. Barton, J. S. Bullock, and C. Q. Trinh. Small-scale Structure in the Sloan Digital Sky Survey and $\Lambda$ CDM: Isolated $\mathrm{L}$ * Galaxies with Bright Satellites. ApJ, 738:102, Sept. 2011.

375 W. H. Trick, J. Bovy, E. D’Onghia, and H.-W. Rix. Action-based Dynamical Modeling for the Milky Way Disk: The Influence of Spiral Arms. ApJ, 839:61, Apr. 2017.

376 W. H. Trick, J. Bovy, and H.-W. Rix. Action-Based Dynamical Modeling for the Milky Way Disk. ApJ, 830:97, Oct. 2016.

377 H. C. van de Hulst, C. A. Muller, and J. H. Oort. The spiral structure of the outer part of the Galactic System derived from the hydrogen emission at $21 \mathrm{~cm}$ wavelength. Bull. Astron. Inst. Netherlands, 12:117, May 1954.

378 R. P. van der Marel, J. Anderson, A. Bellini, G. Besla, P. Bianchini, M. Boylan-Kolchin, J. Chaname, A. Deason, and et al. Local Group and Star Cluster Dynamics from HSTPROMO: The Hubble Space Telescope Proper Motion Collaboration. In M. S. Seigar and P. Treuthardt, editors, Structure and Dynamics of Disk Galaxies, volume 480 of Astronomical Society of the Pacific Conference Series, page 43, Mar 2014.

379 R. P. van der Marel, G. Besla, T. J. Cox, S. T. Sohn, and J. Anderson. The M31 Velocity Vector. III. Future Milky Way M31-M33 Orbital Evolution, Merging, and Fate of the Sun. ApJ, 753(1):9, Jul 2012.

380 R. P. van der Marel, M. Fardal, G. Besla, R. L. Beaton, S. T. Sohn, J. Anderson, T. Brown, and P. Guhathakurta. The M31 Velocity Vector. II. Radial Orbit toward the Milky Way and Implied Local Group Mass. ApJ, 753:8, July 2012.

381 A. Varghese, R. Ibata, and G. F. Lewis. Stellar streams as probes of dark halo mass and morphology: a Bayesian reconstruction. MNRAS, 417:198-215, Oct. 2011.

382 E. Vasiliev. AGAMA: Action-based galaxy modeling framework. Astrophysics Source Code Library, May 2018.

383 E. Vasiliev. Proper motions and dynamics of the Milky Way globular cluster system from Gaia DR2. MNRAS, 484:2832-2850, Apr. 2019. 
384 C. Vera-Ciro and A. Helmi. Constraints on the Shape of the Milky Way Dark Matter Halo from the Sagittarius Stream. ApJ, 773:L4, Aug. 2013.

385 M. Vogelsberger, S. Genel, V. Springel, P. Torrey, D. Sijacki, D. Xu, G. Snyder, S. Bird, D. Nelson, and L. Hernquist. Properties of galaxies reproduced by a hydrodynamic simulation. Nature, 509:177-182, May 2014.

386 M. Vogelsberger, S. Genel, V. Springel, P. Torrey, D. Sijacki, D. Xu, G. Snyder, D. Nelson, and et al. Introducing the Illustris Project: simulating the coevolution of dark and visible matter in the Universe. MNRAS, 444:1518-1547, Oct. 2014.

387 M. G. Walker, M. Mateo, E. W. Olszewski, J. Peñarrubia, N. W. Evans, and G. Gilmore. A Universal Mass Profile for Dwarf Spheroidal Galaxies? ApJ, 704(2):1274-1287, Oct 2009.

388 J. Wang, C. S. Frenk, J. F. Navarro, L. Gao, and T. Sawala. The missing massive satellites of the Milky Way. MNRAS, 424(4):2715-2721, Aug 2012.

389 J. Wang, J. F. Navarro, C. S. Frenk, S. D. M. White, V. Springel, A. Jenkins, A. Helmi, A. Ludlow, and M. Vogelsberger. Assembly history and structure of galactic cold dark matter haloes. MNRAS, 413:13731382, May 2011.

390 W. Wang, J. Han, S. Cole, C. Frenk, and T. Sawala. What to expect from dynamical modelling of galactic haloes. MNRAS, 470:2351-2366, Sept. 2017.

391 W. Wang, J. Han, S. Cole, S. More, C. Frenk, and M. Schaller. What to expect from dynamical modelling of galactic haloes - II. The spherical Jeans equation. MNRAS, 476:5669-5680, June 2018.

392 W. Wang, J. Han, A. P. Cooper, S. Cole, C. Frenk, and B. Lowing. Estimating the dark matter halo mass of our Milky Way using dynamical tracers. MNRAS, 453:377-400, Oct. 2015.

393 L. L. Watkins, N. W. Evans, and J. H. An. The masses of the Milky Way and Andromeda galaxies. MNRAS, 406:264-278, July 2010.

394 L. L. Watkins, N. W. Evans, V. Belokurov, M. C. Smith, P. C. Hewett, D. M. Bramich, G. F. Gilmore, M. J. Irwin, and et al. Substructure revealed by RRLyraes in SDSS Stripe 82. MNRAS, 398:1757-1770, Oct. 2009.
395 L. L. Watkins, R. P. van der Marel, S. T. Sohn, and N. W. Evans. Evidence for an Intermediate-mass Milky Way from Gaia DR2 Halo Globular Cluster Motions. ApJ, 873:118, Mar. 2019.

396 M. Weber and W. de Boer. Determination of the local dark matter density in our Galaxy. A\&A, 509:A25, Jan 2010 .

397 J. K. Werk, J. X. Prochaska, J. Tumlinson, M. S. Peeples, T. M. Tripp, A. J. Fox, N. Lehner, C. Thom, J. M. O’Meara, A. B. Ford, R. Bordoloi, N. Katz, N. Tejos, B. D. Oppenheimer, R. Davé, and D. H. Weinberg. The COS-Halos Survey: Physical Conditions and Baryonic Mass in the Low-redshift Circumgalactic Medium. ApJ, 792:8, Sept. 2014.

398 S. D. M. White and M. J. Rees. Core condensation in heavy halos - A two-stage theory for galaxy formation and clustering. MNRAS, 183:341-358, May 1978.

399 L. M. Widrow. Distribution Functions for Cuspy Dark Matter Density Profiles. ApJS, 131:39-46, Nov. 2000.

400 M. I. Wilkinson and N. W. Evans. The present and future mass of the Milky Way halo. MNRAS, 310:645662, Dec. 1999.

401 A. A. Williams, V. Belokurov, A. R. Casey, and N. W. Evans. On the run: mapping the escape speed across the Galaxy with SDSS. MNRAS, 468(2):2359-2371, Jun 2017.

402 A. A. Williams and N. W. Evans. Haloes light and dark: dynamical models of the stellar halo and constraints on the mass of the Galaxy. MNRAS, 454:698-707, Nov. 2015.

403 A. A. Williams and N. W. Evans. Made-to-measure dark matter haloes, elliptical galaxies and dwarf galaxies in action coordinates. MNRAS, 448:1360-1371, Apr. 2015.

404 A. A. Williams, N. W. Evans, and A. D. Bowden. Hamiltonians of spherical Galaxies in action-angle coordinates. MNRAS, 442:1405-1410, Aug. 2014.

405 M. E. K. Williams, M. Steinmetz, S. Sharma, J. BlandHawthorn, R. S. de Jong, G. M. Seabroke, A. Helmi, K. C. Freeman, and et al. The Dawning of the Stream of Aquarius in RAVE. ApJ, 728:102, Feb. 2011.

406 R. Wojtak, E. L. Łokas, G. A. Mamon, and S. Gottlöber. The mass and anisotropy profiles of galaxy clusters from the projected phase-space density: testing the 
method on simulated data. MNRAS, 399:812-821, Oct. 2009.

407 R. Wojtak, E. L. Łokas, G. A. Mamon, S. Gottlöber, A. Klypin, and Y. Hoffman. The distribution function of dark matter in massive haloes. MNRAS, 388:815828, Aug. 2008.

408 E. L. Wright, P. R. M. Eisenhardt, A. K. Mainzer, M. E. Ressler, R. M. Cutri, T. Jarrett, J. D. Kirkpatrick, D. Padgett, and et al. The Wide-field Infrared Survey Explorer (WISE): Mission Description and Initial Onorbit Performance. AJ, 140:1868-1881, Dec. 2010.

409 M. S. Xiang, X. W. Liu, H. B. Yuan, Y. Huang, Z. Y. Huo, H. W. Zhang, B. Q. Chen, H. H. Zhang, N. C. Sun, C. Wang, Y. H. Zhao, J. R. Shi, A. L. Luo, G. P. Li, Y. Wu, Z. R. Bai, Y. Zhang, Y. H. Hou, H. L. Yuan, G. W. Li, and Z. Wei. The LAMOST stellar parameter pipeline at Peking University - LSP3. MNRAS, 448(1):822-854, Mar 2015.

410 X.-X. Xue, H.-W. Rix, Z. Ma, H. Morrison, J. Bovy, B. Sesar, and W. Janesh. The Radial Profile and Flattening of the Milky Way's Stellar Halo to $80 \mathrm{kpc}$ from the SEGUE K-giant Survey. ApJ, 809(2):144, Aug 2015.

411 X. X. Xue, H. W. Rix, G. Zhao, P. Re Fiorentin, T. Naab, M. Steinmetz, F. C. van den Bosch, T. C. Beers, and et al. The Milky Way's Circular Velocity Curve to $60 \mathrm{kpc}$ and an Estimate of the Dark Matter Halo Mass from the Kinematics of 2400 SDSS Blue Horizontal-Branch Stars. ApJ, 684:1143-1158, Sept. 2008.

412 B. Yanny, H. J. Newberg, S. Kent, S. A. LaurentMuehleisen, J. R. Pier, G. T. Richards, C. Stoughton, J. E. Anderson, Jr., and et al. Identification of A-colored Stars and Structure in the Halo of the Milky Way from Sloan Digital Sky Survey Commissioning Data. ApJ, 540:825-841, Sept. 2000.

413 B. Yanny, C. Rockosi, H. J. Newberg, G. R. Knapp, J. K. Adelman-McCarthy, B. Alcorn, S. Allam, C. Allende Prieto, and et al. SEGUE: A Spectroscopic Survey of 240,000 Stars with $g=14-20$. AJ, 137:43774399, May 2009.

414 J. H. Yoon, K. V. Johnston, and D. W. Hogg. Clumpy Streams from Clumpy Halos: Detecting Missing Satellites with Cold Stellar Structures. ApJ, 731:58, Apr. 2011.
415 D. G. York, J. Adelman, J. E. Anderson, Jr., S. F. Anderson, J. Annis, N. A. Bahcall, J. A. Bakken, R. Barkhouser, , and SDSS Collaboration. The Sloan Digital Sky Survey: Technical Summary. AJ, 120:1579-1587, Sept. 2000.

416 Q. Yu and P. Madau. Kinematics of hypervelocity stars in the triaxial halo of the Milky Way. MNRAS, 379:1293-1301, Aug. 2007.

417 Q. Yu and S. Tremaine. Ejection of Hypervelocity Stars by the (Binary) Black Hole in the Galactic Center. ApJ, 599:1129-1138, Dec. 2003.

418 Z. Yuan, J. Chang, P. Banerjee, J. Han, X. Kang, and M. C. Smith. StarGO: A New Method to Identify the Galactic Origins of Halo Stars. ApJ, 863(1):26, Aug 2018.

419 Z. Yuan, G. C. Myeong, T. C. Beers, N. W. Evans, Y. S. Lee, P. Banerjee, D. Gudin, K. Hattori, H. Li, T. Matsuno, V. M. Placco, M. C. Smith, D. D. Whitten, and G. Zhao. Dynamical Relics of the Ancient Galactic Halo. arXiv e-prints, page arXiv:1910.07538, Oct 2019.

420 D. Zaritsky, C. Conroy, H. Zhang, R. P. Naidu, A. Bonaca, N. Caldwell, P. A. Cargile, and B. D. Johnson. A Lower Limit on the Mass of Our Galaxy from the H3 Survey. ApJ, 888(2):114, Jan 2020.

421 D. Zaritsky and H. Courtois. A dynamics-free lower bound on the mass of our Galaxy. MNRAS, 465:37243728, Mar. 2017.

422 D. Zaritsky, E. W. Olszewski, R. A. Schommer, R. C. Peterson, and M. Aaronson. Velocities of stars in remote Galactic satellites and the mass of the Galaxy. ApJ, 345:759-769, Oct. 1989.

423 M. Zhai, Q. Guo, G. Zhao, Q. Gu, and A. Liu. Local Group Analogs in $\Lambda$ CDM Cosmological Simulations. ApJ, 890(1):27, Feb 2020.

424 M. Zhai, X.-X. Xue, L. Zhang, C.-D. Li, G. Zhao, and C.-Q. Yang. The mass of the Galactic dark matter halo from 9000 LAMOST DR5 K giants. Research in Astronomy and Astrophysics, 18(9):113, Aug 2018.

425 H.-X. Zhang, E. W. Peng, P. Côté, C. Liu, L. Ferrarese, J.-C. Cuillandre, N. Caldwell, S. D. J. Gwyn, and et al. The Next Generation Virgo Cluster Survey. VI. The Kinematics of Ultra-compact Dwarfs and Globular Clusters in M87. ApJ, 802(1):30, Mar 2015. 
426 H.-X. Zhang, T. H. Puzia, E. W. Peng, C. Liu, P. Côté, L. Ferrarese, P.-A. Duc, P. Eigenthaler, and et al. Stellar Population Properties of Ultracompact Dwarfs in M87: A Mass-Metallicity Correlation Connecting Lowmetallicity Globular Clusters and Compact Ellipticals. ApJ, 858(1):37, May 2018.

427 L. Zhang, H.-W. Rix, G. van de Ven, J. Bovy, C. Liu, and G. Zhao. The Gravitational Potential near the Sun from SEGUE K-dwarf Kinematics. ApJ, 772:108, Aug. 2013.

428 G. Zhao, Y.-H. Zhao, Y.-Q. Chu, Y.-P. Jing, and L.C. Deng. LAMOST spectral survey - An overview. Research in Astronomy and Astrophysics, 12:723-734, July 2012.

429 L. Zhu, R. van den Bosch, G. van de Ven, M. Lyubenova, J. Falcón-Barroso, S. E. Meidt, M. Martig, J. Shen, Z.-Y. Li, A. Yildirim, C. J. Walcher, and S. F. Sanchez. Orbital decomposition of CALIFA spiral galaxies. MNRAS, 473(3):3000-3018, Jan 2018.

\section{Appendix A MW mass measurements}


Table A1 MW mass measurements

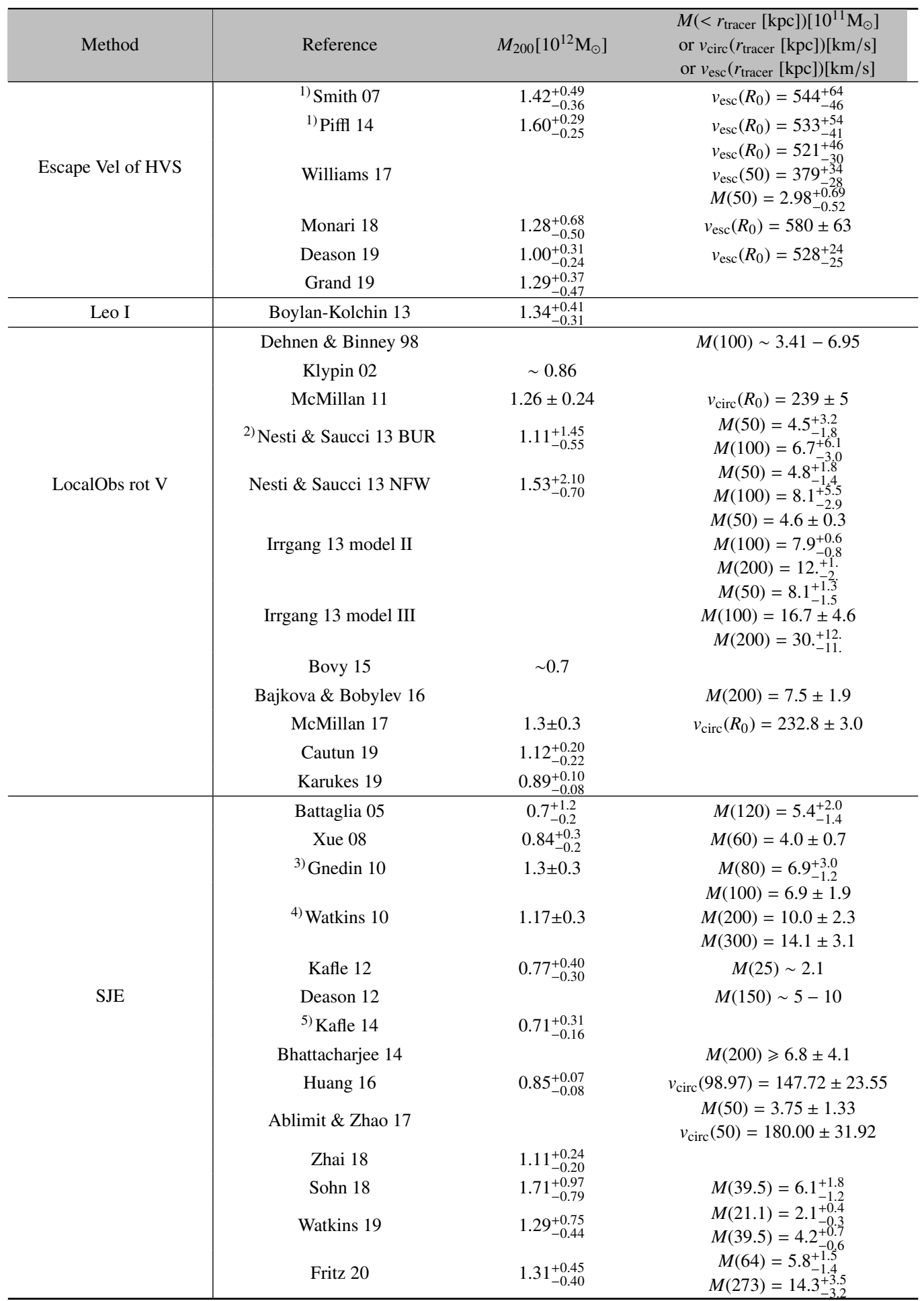

\footnotetext{
1) The error of the escape velocity is $90 \%$ confidence region.

2) The original 2- $\sigma$ errors are shrinked by $10 \%$.

3) The virial radius was adopted to be $300 \mathrm{kpc}$.

4) The virial radius was adopted to be $300 \mathrm{kpc}$.

If considering systematic uncertainties due to velocity anisotropy, $\beta$, the error becomes $M_{200}=1.17_{-0.20}^{+1.30} \mathrm{M}_{\odot}$.

${ }^{5)}$ Velocity dispersion measured on K giants out to $160 \mathrm{kpc}$.
} 


\begin{tabular}{|c|c|c|c|}
\hline Method & Reference & $M_{200}\left[10^{12} \mathrm{M}_{\odot}\right]$ & $\begin{array}{l}M\left(<r_{\text {tracer }} \mathrm{kpc}\right)\left[10^{11} \mathrm{M}_{\odot}\right] \\
\text { or } v_{\text {circ }}\left(r_{\text {tracer }}[\mathrm{kpc}]\right)[\mathrm{km} / \mathrm{s}]\end{array}$ \\
\hline \multirow{18}{*}{ DF } & ${ }^{6)}$ Kochanek 96 w Leo I & & $M(50)=5.1_{-1.1}^{+1.3}$ \\
\hline & ${ }^{6)}$ Kochanek 96 no Leo I & & $M(50)=3.9_{-0.7}^{+1.6}$ \\
\hline & 7) Wilkinson \& Evans 99 & $1.9_{-1.7}^{+3.6}$ & $M(50)=5.4_{-3.6}^{+0.1}$ \\
\hline & 7) Sakamoto 03 w Leo I & $2.5_{-1.0}^{+0.5}$ & $M(50)=5.5_{-0.2}^{+0.0}$ \\
\hline & Sakamoto 03 no Leo I & $1.8_{-0.7}^{+0.4}$ & $M(50)=5.4_{-0.4}^{+0.1}$ \\
\hline & Deason $12 b$ & $0.94_{-0.20}^{+0.22}$ & $M(50)=4.2 \pm 0.4$ \\
\hline & 8) Eadie 15 & $1.55_{-0.13}^{+0.18}$ & $M(260)=13.7_{-1.0}^{+1.4}$ \\
\hline & 8) Eadie 15 no Pal 3 & $1.36_{-0.10}^{+0.15}$ & \\
\hline & Williams \& Evans 15 & & $M(50) \sim 4.5$ \\
\hline & 8) Eadie 16 & $0.68_{-0.08}^{+0.07}$ & $M(125)=5.22_{-0.43}^{+0.41}$ \\
\hline & 8) Eadie 16 no $<10 \mathrm{kpc} \mathrm{GC}$ & $0.90_{-0.33}^{+0.18}$ & \\
\hline & 8) Eadie 17 & $0.86_{-0.19}^{+0.23}$ & $M(125)=6.3 \pm 1.1$ \\
\hline & Posti 19 & $1.11 \pm 0.30$ & $\begin{aligned} M(20, \text { total }) & =1.91_{-0.15}^{+0.17} \\
M(20, \mathrm{DM}) & =1.37_{-0.11}^{+0.12}\end{aligned}$ \\
\hline & 8) Eadie \& Jurić 19 & $0.77_{-0.16}^{+0.25}$ & $\begin{array}{l}M(25)=2.6_{-0.6}^{+1.0} \\
M(50)=3.7_{-0.8}^{+1.4} \\
M(100)=5.3_{-1.2}^{+2.1}\end{array}$ \\
\hline & 9) Vasiliev 19 & $1.0_{-0.5}^{+1.5}$ & $\begin{array}{c}M(50)=5.4_{-0.8}^{+1.1} \\
M(100)=8.5_{-2.0}^{+3.3}\end{array}$ \\
\hline & Callingham 19 & $1.17_{-0.15}^{+0.21}$ & \\
\hline & Li 19 & $1.23_{-0.18}^{+0.21}$ & \\
\hline & Li 19 with rotation curve & $1.26_{-0.15}^{+0.17}$ & \\
\hline \multirow{10}{*}{ stream } & Lin 95 & & $M(100) \sim 5.5 \pm 1.0$ \\
\hline & Law 05 & & $M(50) \sim 3.8-5.6$ \\
\hline & Koposov 10 & & $v_{\text {circ }}\left(R_{0}\right)=221 \pm 18$ \\
\hline & Newberg 10 & & $M(60) \sim 2.7$ \\
\hline & ${ }^{10)}$ Gibbons 14 & $0.56 \pm 0.12$ & $\begin{array}{c}M(50)=2.9 \pm 0.5 \\
M(100)=4.1 \pm 0.7\end{array}$ \\
\hline & Küpper 15 & $1.69 \pm 0.42$ & $\begin{array}{l}M(19, \text { Galaxy })=2.14_{-0.35}^{+0.38} \\
\quad v_{\text {circ }}\left(R_{0}\right)=253 \pm 16\end{array}$ \\
\hline & Malhan \& Ibata 19 & & $M(20)=2.5 \pm 0.2$ \\
\hline & & & $v_{\text {circ }}\left(R_{0}\right)=244 \pm 4$ \\
\hline & Hendel 18 & & $M(60)<5.6_{-1.1}^{+1.2}$ \\
\hline & Erkal 19 & & $M(50)=3.8_{-0.11}^{+0.14}$ \\
\hline \multirow{7}{*}{ timing \& LG dyn. } & Li \& White 08 & $2.43_{-0.53}^{+0.49}$ & \\
\hline & Sohn 13 & $2.65_{-1.36}^{+1.58}$ & \\
\hline & Diaz 14 & $0.8 \pm 0.5$ & \\
\hline & Peñarrubia 14 & $0.80_{-0.30}^{+0.40}$ & $v_{\text {circ }}\left(R_{0}\right)=245 \pm 23$ \\
\hline & Peñarrubia 16 & $0.87_{-0.19}^{+0.22}$ & \\
\hline & 6) Zaritsky 19 & $0.91-2.13$ & \\
\hline & Zhai 20 & $1.5_{-0.7}^{+1.4}-2.5_{-1.4}^{+2.2}$ & \\
\hline \multicolumn{4}{|c|}{ 6) $90 \%$ confidence region. } \\
\hline \multicolumn{4}{|c|}{ 7) Error includes systematics. } \\
\hline \multicolumn{4}{|c|}{ The virial mass is the flat rotation model parameter. } \\
\hline \multicolumn{4}{|c|}{ 8) $95 \%$ credible regions are quoted for Eadie 15 and 17.} \\
\hline \multicolumn{4}{|c|}{$\begin{array}{l}50 \% \text { credible regions are quoted for Eadie } 16 \text { and for the mass enclosed within } 25,50 \text { and } 100 \mathrm{kpc} \text { by Eadie \& Jurić } \\
19 .\end{array}$} \\
\hline \multicolumn{4}{|c|}{ Error of $M_{200}$ by Eadie \& Juric 19 is the $68 \%$ credible region reading from their figures. } \\
\hline \multicolumn{4}{|c|}{ Eadie 15 estimate is the Hernquist mass. } \\
\hline \multicolumn{4}{|c|}{ Eadie 19 estimate has inner cut of $20 \mathrm{kpc}$. } \\
\hline \multicolumn{4}{|c|}{ 9) Error of $M_{200}$ includes uncertainties in the outer slope of the halo density profile. } \\
\hline
\end{tabular}




\begin{tabular}{|c|c|c|c|}
\hline Method & Reference & $M_{200}\left[10^{12} \mathrm{M}_{\odot}\right]$ & $M\left(<r_{\text {tracer }} \mathrm{kpc}\right)\left[10^{11} \mathrm{M}_{\odot}\right]$ \\
\hline \multirow{8}{*}{ satellite phenomenology } & Busha 11 & $1.0_{-0.4}^{+0.7}$ & \\
\hline & González 13 & $1.15_{-0.34}^{+0.48}$ & \\
\hline & Patel 17 & $0.83_{-0.55}^{+0.77}$ & \\
\hline & Sales 07 & $0.58_{-0.20}^{+0.24}$ & \\
\hline & Barber 14 & $1.10_{-0.29}^{+0.45}$ & \\
\hline & Cautun 14 & $0.78_{-0.33}^{+0.57}$ & \\
\hline & Patel 18 & $0.68_{-0.26}^{+0.23}$ & \\
\hline & Patel 18 no Sagittarius & $0.78_{-0.28}^{+0.29}$ & \\
\hline Dynamics-free & Zaritsky \& Courtois 17 & $\sim 1.2$ & \\
\hline
\end{tabular}

